\title{
TUBULAR LYSOSOME BIOGENESIS IN INNATE IMMUNE CELLS
}

by

\author{
Amra Saric \\ B.Sc., Ryerson University, 2010
}

\begin{abstract}
A Dissertation
presented to Ryerson University
\end{abstract}

in partial fulfillment of the requirements for the degree of

Doctor of Philosophy

in the Program of

Molecular Science

Toronto, Ontario, Canada, 2016

(C)Amra Saric 2016 


\section{AUTHOR'S DECLARATION FOR ELECTRONIC SUBMISSION OF A DISSERTATION}

I hereby declare that I am the sole author of this dissertation. This is a true copy of the dissertation, including any required final revisions, as accepted by my examiners.

I authorize Ryerson University to lend this dissertation to other institutions or individuals for the purpose of scholarly research.

I further authorize Ryerson University to reproduce this dissertation by photocopying or by other means, in total or in part, at the request of other institutions or individuals for the purpose of scholarly research.

I understand that my dissertation may be made electronically available to the public. 


\title{
TUBULAR LYSOSOME BIOGENESIS IN INNATE IMMUNE CELLS \\ Amra Saric \\ Doctor of Philosophy, 2016 \\ Molecular Science, Ryerson University
}

\begin{abstract}
Lysosomes are essential organelles required for breakdown of endocytic and biosynthetic cargo, pathogen killing and autophagy. In most cells, lysosomes are typically small punctate structures. By contrast, innate immune cells like macrophages and dendritic cells that have been exposed to bacterial lipopolysaccharides (LPS) exhibit strikingly tubular lysosomes (TLs) and lysosomerelated major histocompatibility class II (MHCII) compartments (MIIC), respectively. TLs are suggested to play a role in phagosome maturation and retention of fluidphase endocytic uptake in activated macrophages. In addition, the dendritic cell tubular MIIC (tMIIC) may be involved in antigen presentation. Since remarkably little was known about how tubular lysosomes form, I took to investigate the molecular requirements for this process in macrophages and dendritic cells and present my findings in this thesis.
\end{abstract}

Here, I confirm that microtubules are necessary as a template for lysosome tubulation, along with dynein and kinesin microtubule-dependent motors. We were first to identify molecular components necessary for lysosome tubulation; TL biogenesis required the concerted action of the Arl8b GTPase, along with its effector 
SKIP, a kinesin adaptor protein, and the Rab7 GTPase, along with its effectors RILP and FYC01, which are adaptor proteins for dynein and kinesin, respectively. Importantly, we observed that TLs are highly dynamic structures whereas punctate lysosomes are conspicuously more static.

I also present evidence that mTOR, a lysosomal protein kinase, is required for LPS-induced TL biogenesis and cell surface delivery of MHCII in macrophages and dendritic cells. First, I show that the MyD88-PI3K-Akt-mTOR signaling pathway regulates LPS-induced lysosome tubulation. Second, I demonstrate that mTOR is required for anterograde lysosomal transport suggesting that this kinase may regulate tubulation and antigen presentation by modulating the microtubule-based motor activity of lysosomes.

Finally, I present preliminary data on the properties of tubular lysosomes compared to punctate lysosomes in an effort to characterize these organelles. Among the data presented is evidence that total lysosomal volume increases significantly upon tubulation, which may have important underlying implications in antigen sampling and processing.

Overall, my work has expanded on our knowledge of how morphology and trafficking of lysosomes is modulated in immune cells, which may alter cell function. 
Acknowledgements

Firstly, I acknowledge my supervisor and mentor, Dr. Roberto Botelho, whose guidance and passion for science greatly influenced my completion of this body of work. I am deeply appreciative of the time and effort Dr. Botelho has invested in my graduate studies and to shaping my overall positive outlook on research and academia.

I would like to thank Dr. Sergio Grinstein, who has provided invaluable advice and thought-stimulating discussions that have strongly impacted by graduate research projects. I am greatly indebted to Dr. Grinstein for his generosity during our collaboration. In addition, I owe a great deal of gratitude to Dr. Jason G. Kay, who without a doubt has had the strongest impact on my technical training experience.

I am profoundly thankful to all members of the Botelho and Grinstein labs. In particular, I thank Wendy Furuya whose help with my earlier project has been essential to its successful completion. I also thank Drs. Spencer Freeman and Danielle Johnson, Daniel Schlam, Harikesh Wong, Shannon Ho, Christopher Choy, Monica Dayam and Victoria Hipolito, all of whom I have either worked closely with or who have significantly contributed to my progress by means of moral support and stimulating discussions.

I wish to thank my graduate committee, Drs. Debora Foster and Jeffrey Fillingham, and the Molecular Science Graduate Program Director Dr. Michael Arts. The feedback and support from my graduate committee has been of utmost value to me throughout the years. Communication with Dr. Arts has kept me confident and in good spirits during the arduous task of thesis writing and preparation for a defense.

Finally, I thank my mom, dad and sister for their constant love and support. 


\section{Table of Contents}

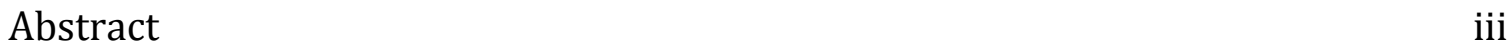

Acknowledgements $\quad$ V

Table of Contents $\quad$ vi

List of Tables $\quad$ ix

List of Figures $\quad$ X

List of Abbreviations $\quad$ xii

Chapter 1: Introduction 1

1.1: Dissertation Overview 2

1.2 Macrophages and Dendritic Cells in Innate Immunity 4

1.2.1 Functions of Macrophages and Dendritic Cells 4

1.2.1.1 Phagocyte Uptake Mechanisms 5

1.2.1.2 Antigen Processing and Presentation by Major Histocompatibility Complex Class II 6

1.2.2 Innate Immune Recognition by Macrophages and DCs $\quad 7$

1.2.2.1 Pattern Recognition Receptors and Microbe-Associated Molecular $\begin{array}{ll}\text { Patterns } & 7\end{array}$

1.2.2.2 TLR4 Signaling 8

1.3 Lysosomal Membrane Trafficking 9

1.3.1 Microtubule-Based Motility 10

1.3.2 Organelle Identity and Transport in the Endocytic Pathway 12

$\begin{array}{ll}\text { 1.3.2.1 Phosphoinositides } & 13\end{array}$

1.3.2.2 The Small GTPases and Effector Proteins 14

$\begin{array}{ll}\text { 1.3.2.2.1 Rab7 and Arl8b } & 15\end{array}$

1.4 Mechanistic Target of Rapamycin and Lysosomes 17

1.4.1 Nutrient Sensing at the Lysosome 19

1.4.2 Regulation of Lysosomal Dynamics by mTOR 20

$\begin{array}{ll}1.5 \text { Lysosome Tubulation } & 21\end{array}$ 
Chapter 2: Experimental Procedures $\quad 37$

2.1: Cell Culture 38

2.2: Transfection and Plasmids 38

2.3: Gene Silencing by siRNA Electroporation 39

2.4: SDS-PAGE and Western Blotting 39

2.5: Pharmacological Inhibition $\quad 40$

2.6 Immunofluorescence $\quad 41$

2.7 Flow Cytometry $\quad 41$

2.8: Reverse Transcription and Quantitative PCR 42

2.9: Lysosome Tubulation Assays 42

2.10: Timelapse Microscopy and Particle Tracking 43

2.11: Lysosomal Positioning Assays 44

2.12 Image Quantification of LC3, RFP-Rab7 and Arl8b-GFP 44

2.13: Statistical Analyses 45

Chapter 3: Results $\quad 46$

3.1: Rab7 and Arl8 GTPases are Necessary for Lysosome Tubulation in $\begin{array}{ll}\text { Macrophages } & 47\end{array}$

3.1.1: LPS-Stimulated Macrophage Cell Lines Form Highly Dynamic Lysosome $\begin{array}{ll}\text { Tubules } & 47\end{array}$

3.1.2: Dynein and Kinesin I are Required for Lysosome Tubulation 48

3.1.3: Rab7 is Necessary for Lysosome Tubulation 49

3.1.4: RILP is Involved in Lysosome Tubulation 49

3.1.5: FYC01 Plays a Role in Lysosome Tubulation 50

3.1.6: Arl8b and SKIP are Necessary for Lysosome Tubulation 51

3.1.7: Lysosome Tubules are More Motile than Punctate Lysosomes 53

3.2: mTOR Regulates Lysosome Tubulation and Antigen Presentation in Macrophages and Dendritic Cells $\quad 55$

3.2.1: TLR4-Induced Lysosome Tubulation in Macrophages Requires MyD88 and PI3-Kinase 
3.2.2: The Canonical PI3K-Akt Pathway Regulates LPS-Induced Lysosome Tubulation

3.2.3: mTOR is Required for LPS-Induced Lysosome Tubulation in Macrophages

3.2.4: The Akt-mTOR Axis Prolongs LPS-Derived Signaling in Macrophages 58

3.2.5: mTOR Controls Lysosome/MIIC Tubulation in Primary Dendritic Cells 59

3.2.6: Autophagy Does Not Impact LPS-Mediated Lysosome Tubulation $\quad 60$

3.2.7: mTOR is Required for AnterogradeLlysosomal Transport 61

3.2.8: mTOR is Required for Antigen Presentation in Dendritic Cells 62

3.2.9: Model of Tubular Lysosome Biogenesis 63

Chapter 4: Discussion $\quad 99$

4.1: Rab7 and Arl8 GTPases are Necessary for Lysosome Tubulation in $\begin{array}{ll}\text { Macrophages } & 100\end{array}$

4.2: mTOR Regulates Lysosome Tubulation and Antigen Presentation in Macrophages and Dendritic Cells

4.3 Preliminary Data and Discussion on the Physiological Properties of Tubular $\begin{array}{ll}\text { Lysosomes } & 108\end{array}$

4.3.1 Trafficking of Endocytic Cargo to Tubular Lysosomes 108

4.3.2 Lysosome Tubulation and Lumenal Volume 109

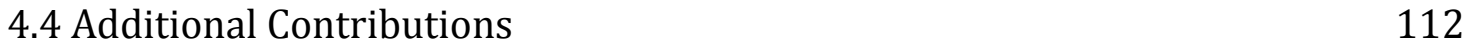

$\begin{array}{ll}\text { Conclusion } & 125\end{array}$

$\begin{array}{ll}\text { Appendix } & 128\end{array}$

$\begin{array}{ll}\text { References } & 135\end{array}$ 


\section{List of Tables}

Table A-1: Absolute values of lysosome tubulation number for section 3.1.

130 


\section{List of Figures}

Figure 1.1: Lysosomal Trafficking Pathways.

Figure 1.2: Antigen Processing and Presentation by MHCII in Antigen Presenting Cells (APCs).

Figure 1.3: TLR4 Signaling Pathways. $\quad 30$

Figure 1.4: Microtubule-Based Organelle Transport. 32

Figure 1.5: Activation Cycle of Small GTPases. 34

Figure 1.6: Lysosomal Inside-out Activation of mTORC1 by Amino Acids. 36

Figure 3.1: LPS Stimulates Lysosome Tubulation in Macrophage Cell Lines. $\quad 66$

Figure 3.2: Dynein and Kinesin I are Required for Lysosome Tubulation. 68

Figure 3.3: Rab7 is Required for Lysosome Tubulaton. 70

Figure 3.4: RILP is Involved in Lysosome Tubulation. 72

Figure 3.5: FYCO1 is Involved in Lysosome Tubulation.

Figure 3.6: Arl8b and SKIP are Required for Lysosome Tubulation. 76

Figure 3.7: Tubular Lysosomes are Highly Motile. 78

Figure 3.8: Schematic of TLR4 Signaling Pathways and Compounds that Selectively Inhibit them. $\quad 80$

Figure 3.9: MyD88 and PI3K are Required for LPS-Induced Lysosome Tubulation in

Macrophages. 82

Figure 3.10: The Canonical PI3K-Akt Pathway Regulates LPS-Induced Lysosome

Tubulation in Macrophages. 84

Figure 3.11: mTOR is Activated by LPS and is Required for Lysosome Tubulation in

Macrophages. 86

Figure 3.12: The Akt-mTOR Pathway is Activated Early and Maintained Under LPS

Signaling and Coincides with Lysosome Tubulation. 88

Figure 3.13: mTOR is Required for MIIC Tubulation in Primary Dendritic Cells. 90

Figure 3.14: Torin1 Blocks Lysosome Tubulation in a Manner Independent of

Autophagy. $\quad 92$

Figure 3.15: mTOR is Required for Anterograde Lysosomal Transport. 94

Figure 3.16: mTOR is Required for LPS-Induced Cell Surface Delivery of MHCII. 96 
Figure 3.17: Model of Tubular Lysosome Biogenesis in Macrophages and Dendritic

Cells. $\quad 98$

Figure 4.1: LPS Increases the Levels of Membrane-Associated Arl8b in an mTORDependent Manner.

Figure 4.2: Endocytic Fluid-Phase Cargo Traffics to Tubular Lysosomes at the Same

Rate as to Punctate Lysosomes. 120

Figure 4.3: Total Lysosomal Volume Increases upon Tubulation. 122

Figure 4.4 Evidence for Membrane Fusion and Fission in Regulating Tubular Lysosome Biogenesis. 124

Figure A-1: MRT67307, IRAK1/4 Inhibitor and PepinhMYD Inhibit Pathways Downstream of their Targets. 131

Figure A-2: mTOR Activity is Required for Lysosome Tubulation. 133

Figure A-3: mTOR Inhibition does not Affect Microtubules or Basal Lysosomal Motility. 134 
Abt1 - activator of basal transcription 1

Ag - antigen

ALR - autophagic lysosome reformation

APC - antigen presenting cell

Arl - Arf-like

Atg13 - autophagy-related protein 13

ATPase - adenosine triphosphatase

BMDC - bone marrow-derived dendritic cell

cDNA - complementary DNA

CLIP-170 - cytoplasmic inker protein of $170 \mathrm{kDa}$

DC - dendritic cell

deptor - DEP domain-containing mTOR-interacting protein

EE - early endosome

EEA1 - early endosomal antigen 1

$\mathrm{Fc} \gamma \mathrm{R}$ - Fc gamma receptor

FYCO1 - FYVE and coiled-coil domain-containing protein 1

FYVE - Fab1, YOTB, Vac1, EEA1

GAP - GTPase activating protein

GDI - GDP dissociation inhibitor

GEF - guanine nucleotide exchange factor

GTPase - guanosine triphosphatase

IgG - immunoglobulin G

IkB - inhibitor of kappa B

IKK - IkB kinase

IL-6 - interleukin-6

IRAK1/4 - interleukin-1 receptor-associated kinase 1/4

IRF3 - interferon regulatory factor 3

KIF - kinesin family member

KIF5B - kinesin family member 5B / conventional kinesin / kinesn I 
LAMP1 - lysosome-associated membrane protein 1

LC3 - light Chain 3

LE - late endosome

LPS - lipopolysaccharide

MAL - MyD88 adaptor-like

MAMP - microbe-associated molecular pattern

MHCII - major histocompatibility complex class II

MIIC - MHCII compartment

mLST8 - mammalian lethal with SEC13 8

mSin1 - stress-activated MAP-kinase interacting protein 1

MT - microtubule

MTOC - microtubule organizing center

mTOR - mechanistic target of rapamycin

mTORC1/mTORC2 - mTOR complex 1/2

MVB - multivesicular body

MyD88 - myeloid differentiation primary response protein 88

NFkB - nuclear factor kappa B

NPC1 - Niemann-Pick C1

ORP1L - oxysterol-binding protein-related protein 1L

PepinhCtrl - control peptide

PepinhMYD - peptide inhibitor of MyD88

PH - pleckstrin homology

PI - phosphatidylinositol

PI3K - phosphatidylinositol 3-kinase

PIP - phosphoinositide

PRAS40 - proline-rich Akt1 substrate

protor1/2 - protein observed with rictor

PRR - pattern recognition receptor

qRT-PCR/qPCR - quantitative real-time polymerase chain reaction

raptor - regulatory associated protein of mTOR

Rheb - Ras homolog enriched in the brain 
rictor - rapamycin insensitive component of TOR

RILP - Rab7-interacting lysosomal protein

SDS-PAGE - sodium dodecyl sulfate-polyacrylamide gel electrophoresis

Ser/Thr - serine/threonine

siRNA - small interfering RNA

SKIP - SifA and kinesin-interacting protein

TBK1 - TANK-binding kinase 1

tel2 - telomere length regulation protein 2

TL - tubular lysosome

TLR - toll-like receptor

tMIIC - tubular MHCII compartment

TRAF6 - TNF receptor-associated factor 6

TRAM - TRIF-related adaptor molecule

TRIF - TIR-domain containing adaptor inducing interferon- $\beta$

tti1 - tel2-interacting protein 1

Ulk1 - Unc51-like autophagy activating kinase 1

V-ATPase - vacuolar $\mathrm{H}^{+}$-ATPase 
Chapter 1

Introduction 


\section{1: Dissertation Overview}

In this thesis, I present and discuss findings from my research on the molecular requirements of lysosome tubulation in macrophages and dendritic cells.

In Chapter 1, I introduce macrophages and dendritic cells and their roles in innate immunity. As host sentinels, they function to engulf and digest foreign particles by various uptake mechanisms such as phagocytosis, thus neutralizing the immediate threat of invading pathogens. Digestion of foreign matter by these cells confers a unique ability to generate and present antigens on their cell surface to alert the immune system. In addition to these functions, I introduce their means of activation by bacterial molecules via a repertoire of innate immune receptors. Furthermore, I introduce key concepts of membrane trafficking with a focus on lysosomes. The endocytic pathway is discussed in some detail and the major groups of molecules that cells use to confer organelle identity and carry out membrane trafficking are discussed. These include but are not limited to the phosphoinositides, small GTPases and their effectors, microtubules and microtubule-based motor proteins. I focus on lysosome-specific markers and introduce the emerging role of the lysosomal Ser/Thr kinase mTOR as a major sensor of cell stress and metabolism. Finally, I discuss the phenomenon of lysosome tubulation, proposed functions in innate immune cells and the scarcity of knowledge of their biogenesis and regulation. Chapter 1 concludes with rationale and objectives for this thesis.

In Chapter 2, I provide descriptions of the experimental procedures involved in carrying out this research. Techniques include and are not limited to culture of murine cell line macrophages, dendritic cell harvest from mouse bone marrow, plasmid transfection of cells and live-cell confocal fluorescence microscopy. In addition, molecular biology techniques like small interfering RNA (siRNA) gene silencing and quantitative polymerase chain reaction (qPCR) were employed to knock down or detect gene expression levels, respectively. Finally, various assays were developed such as quantification of lysosomal tubulation and intracellular lysosomal positioning. 
Chapter 3 covers the major results of this thesis work presented in two sections: 1) identification of molecular regulators of lysosome tubulation in macrophages, 2) the role of mTOR in lysosome tubulation and antigen presentation in macrophages and dendritic cells. My first project resulted in the identification of multiple membrane trafficking components necessary for lysosome tubulation. Among these components are microtubules, the microtubule-associated motor proteins dynein and kinesin, the lysosomal small GTPases Rab7 and Arl8b as well as their effectors which link to motor proteins. This work was published in the journal Traffic (Mrakovic, Kay, Furuya, Brumell, \& Botelho, 2012). My second major thesis project describes a role for the lysosomal protein kinase mTOR (mechanistic Target of Rapamycin) in lysosome tubulation and antigen presentation, and I provide evidence that mTOR affects anterograde lysosomal transport. A manuscript of this work is in press in the journal Molecular Biology of the Cell (manuscript \# E15-050272).

In Chapter 4, I present a discussion of the two projects grouped into sections mirroring those in Chapter 3. In the first project, I envision that lysosomal morphology is controlled by microtubule-associated molecular machinery that links to lysosomes and pulls the organelle bi-directionally to cause tubulation. In the second project, I discuss the consideration that mTOR may control lysosomal morphology and cell-surface antigen delivery by integrating signals from the LPS receptor TLR4 to act on the microtubule-based transport machinery of this organelle. In addition to these two sections, I include a third, showcasing preliminary data of ongoing projects and/or data I have provided as a secondary contributor to alternate projects. I present a short discussion of each of these contributions.

Finally, I provide concluding remarks on the impact of this thesis in the fields of intracellular membrane trafficking, organelle identity and innate immune cell function. 
1.2 Macrophages and Dendritic Cells in Innate Immunity

All eukaryotic organisms possess some form of immunity. Higher eukaryotes, including mice and humans, have two major immune systems that differ in specificity and outcome: innate and adaptive immune systems. Both work in concert to defend the host organism against invading pathogens and potentially associated infection/disease. The innate immune system is the first line of defense should physical barriers such as the epithelium be breached by foreign microbes, and is a general non-specific response. This system is comprised of specialized cells called leukocytes, which are of myeloid origin and include neutrophils, macrophages and dendritic cells (DCs). While neutrophils have a robust and short-lived response during infection, macrophages and DCs carry out constant surveillance of the host. These cells engulf and kill microbes, clear dead cells and debris from host tissue, and particularly in the case of DCs, bridge innate and adaptive immunity by antigen presentation to $\mathrm{T}$ cells in order to confer a complete immunological response upon the host (Steinman \& Hemmi, 2006). Macrophages and DCs are able to fulfill these diverse roles owing to their numerous functions and methods of innate immune sensing.

\subsubsection{Functions of Macrophages and Dendritic Cells}

The main functions of macrophages and DCs are their abilities to sample the extracellular milieu via several uptake mechanisms, most notably phagocytosis, and in so doing monitor their environment for infection. An overview of uptake mechanisms is presented in Figure 1.1 in the Figures section at the end of this chapter. Particles that are taken up by these cells are trafficked to lysosomes, highly degradative organelles that break down macromolecules into smaller products known as antigens (Watts, 1997). Interestingly, these cells express a set of molecules of the major histocompatibility complex class II (MHCII), which are localized to lysosomes and can bind a vast diversity of antigens (Robinson \& Delvig, 
2002). A critical function of these cells, in particular DCs, is the delivery of MHCIIantigen complexes from lysosomes to the plasma membrane for recognition by $\mathrm{T}$ cells. $\mathrm{T}$ cells then coordinate appropriate adaptive immune responses. An overview of these critical functions is presented in this section.

\subsubsection{Phagocyte Uptake Mechanisms}

In addition to constitutively conducting endocytosis for nutrient uptake as most other cells do, macrophages and DCs are endowed with phagocytic receptors that allow these cells to engulf large particles ( $\geq 0.5 \mu \mathrm{m}$ ) by phagocytosis (Figure 1.1B). For this reason, these cells are professional phagocytes. Of note, immature DCs which have not encountered dangerous particles like pathogenic bacteria, are particularly efficient at macropinocytosis (Steinman \& Swanson, 1995), another uptake process in which large plasma membrane ruffles extend into the extracellular space and collapse to form large intracellular organelles, macropinosomes, full of extracellular fluid (Figure 1.1C). In this way, immature DCs are extremely effective at sampling their environment for sources of antigen. While multiple important uptake mechanisms exist, Fc receptor-mediated phagocytosis, a specialized mechanism largely restricted to these cell types, is introduced here.

Phagocytosis of Immunoglobulin G (IgG) opsonized particles is the most studied of phagocytic processes and will be briefly described here, though there are numerous forms of phagocytosis customized for uptake of numerous types of target particles (Flannagan, Jaumouillé, \& Grinstein, 2012). Particle binding occurs through recognition of the Fc portion of IgG by Fc $\gamma$ receptors on the cell surface of professional phagocytes. Binding of antibody-coated bacteria by these receptors signals to remodel the phagocyte actin cytoskeleton required for formation of pseudopods around the particle for engulfment. Once internalized, particles reside within early phagosomes, organelles with a lumen reminiscent of the extracellular milieu. However, subsequent membrane fusion with endosomes and lysosomes, termed "phagosome maturation" (Beron, Alvarez-Dominguez, Mayorga, \& Stahl, 
1995; Tjelle, Lovdal, \& Berg, 2000), endows phagosomes with numerous microbicidal properties including a lower $\mathrm{pH}$ and enhanced enzymatic activity. Thus fusion with lyososmes not only allows for microbial killing but also allows for generation of antigens through digestion of the foreign particle into smaller molecules. Fittingly, the antigen-presenting MHCII molecules also reside within this compartment. Phagosome maturation and cargo trafficking to the lysosome will be described in more detail in section 1.3, which introduces key concepts of endosomal/lysosomal trafficking.

1.2.1.2 Antigen Processing and Presentation by Major Histocompatibility Complex Class II

In addition to being professional phagocytes, macrophages and DCs are also professional antigen presenting cells (APCs). Indeed, DCs are hailed as the most potent APCs in the body. These cells synthesize MHCII molecules that are composed of $\alpha$ and $\beta$ heterodimers and contain a highly polymorphic region known as the antigen-binding groove. This region is capable of binding an enormous variety of foreign peptides. New MHCII molecules are synthesized along with an endogenous peptide called invariant chain, which blocks the antigen-binding groove during trafficking to endosomes, and thus prevents premature loading of antigen onto MHCII (Roche \& Cresswell, 1990; Cresswell, 1996). The topology of MHCII molecules when in endosomes is such that the antigen-binding groove faces into the lysosomal lumen where antigens can easily be loaded by virtue of their affinity for the groove. As previously discussed, cargo (ie. pathogen) is trafficked to lysosomes and digested to generate small antigenic molecules (Figure 1.1 and Figure 1.2). Protein antigens are processed by lysosomal cathepsins, proteases that function optimally at low $\mathrm{pH}$ and cleave proteins at specific amino acid residues to generate peptides between 15 and 24 amino acids in length. In addition to antigen, invariant chain is also cleaved by acid hydrolases, leaving a small fragment, CLIP (Class II associated invariant chain peptide) within the MHCII peptide-binding groove. An MHCII-like molecule, HLA-DM (human leukocyte antigen-DM), catalyzes the release 
of CLIP from MHCII, and the antigen-binding groove is quickly occupied by a higher affinity antigen (Sloan et al., 1995).

Once antigen is loaded onto MHCII within this lysosome-related MHCII compartment (MIIC), mature DCs deliver large amounts of antigen-MHCII complexes to their plasma membrane (Cella, Engering, Pinet, Pieters, \& Lanzavecchia, 1997). This surface display is necessary for recognition by CD4+ $\mathrm{T}$ helper cells, which directly interact with the MHCII-peptide complex through the Tcell receptor and the co-receptor CD4 (Figure 1.2). Antigen presentation is therefore critical for activating $\mathrm{T}$ cells which further trigger adaptive immunity (Askew, Gatewood, Olivas, Havenith, \& Walker, 1995; Banchereau \& Steinman, 1998; Yrlid \& Wick, 2002). In addition to increasing plasma membrane MHCII, DC maturation is accompanied by a rapid down-regulation of uptake mechanisms and an up-regulation of factors that aid the cell in migration toward lymph nodes, where T cells reside (Banchereau \& Steinman, 1998; Cella, Sallusto, \& Lanzavecchia, 1997).

\subsubsection{Innate Immune Recognition by Macrophages and DCs}

Macrophages and DCs express an array of receptors that recognize and bind common bacterial and viral molecules and thus represent the initial point of discrimination between self and non-self. Collectively, these receptors are termed pattern recognition receptors (PRRs) and the molecules they recognize are termed microbe-associated molecular patterns (MAMPs).

\subsubsection{Pattern Recognition Receptors and Microbe-Associated Molecular Patterns}

The PRRs of macrophages and DCs recognize key evolutionarily conserved microbial signature molecules such as lipopolysaccharide (LPS), flagellin, peptidoglycan and unmethylated CpG DNA. PRRs can be located at the plasma membrane, endosomes or even in the cytosol as is the case with several PRRs dedicated to anti-viral responses. The most important family of PRRs is the Toll-like 
receptors (TLRs), of which there are 10 members in humans and 13 in mice. These receptors are either endosomal or found on the cell surface, where they bind MAMPs, undergo a conformational change and initiate a signaling cascade that ultimately generates an anti-microbial response, such as transcription of proinflammatory genes (Yamamoto \& Takeda, 2010).

TLRs dimerize upon ligand binding and this dimerization can be hetero- or homotypic depending on the ligand and TLR in context. Importantly, dimerization leads to the interaction of intracellular TIR (Toll-IL-1 receptor) domains on the individual TLR molecules, which induces signaling by the receptors by binding to TIR domains of cytoplasmic adaptor proteins. There are four known TLR adaptor proteins: MyD88 (Myeloid differentiation primary response protein 88), MAL (MyD88 adaptor-like), TRIF (TIR domain-containing adaptor-inducing IFN- $\beta$ ) and TRAM (TRIF-related adaptor molecule) (reviewed in O'Neill \& Bowie, 2007). TLRs can signal through any of these adaptors or combinations thereof.

\subsubsection{TLR4 Signaling}

Innate immune cells respond to LPS through TLR. LPS first binds LBP (LPS binding protein) in the serum. CD14 on the surface of leukocytes then transfers the LPS-LBP complex to TLR4, which itself is tightly bound to another molecule critical for its activation, MD2 (Myeloid differentiation factor 2) (Shimazu et al., 1999; Muta \& Takeshige, 2001). Typically, TLR4 activation triggers signaling pathways that result in the production of inflammatory mediators (Figure 1.3, middle and right pathways). A MyD88-independent pathway requires TRIF to activate the kinases TBK1 (TANK binding kinase 1) and IKK (IkB kinase), which stimulate the transcription factor IRF3 (Interferon regulatory factor 3) for the production of type 1 interferons (Fitzgerald et al., 2003; Kawai et al., 2001; Kawai, Adachi, Ogawa, Takeda, \& Akira, 1999; Yamamoto, Sato, \& Hemmi, 2003; Yamamoto et al., 2002; Yamamoto, Sato, Hemmi, et al., 2003). In comparison, the MyD88-dependent pathway activates IRAK1/4 (IL-1 receptor associated kinase) and TRAF6 (TNF 
receptor-associated factor 6) via MyD88 (Burns et al., 2003; Suzuki et al., 2002). Ultimately, this leads to the degradation of IкB (Inhibitor of kappa B), which liberates the bound transcription factor NF- $\mathrm{BB}$ (nuclear factor kappa B) for translocation to the nucleus where it induces production of pro-inflammatory cytokines (Beg, Finco, Nantermet, \& Baldwin, 1993).

LPS-induced stimulation of MyD88 may also activate phosphatidylinositol 3kinase (PI3K), which synthesizes phosphatidylinositol-3,4,5-trisphosphate $\left[\mathrm{PI}(3,4,5) \mathrm{P}_{3}\right]$, a major signaling hub that coordinates cell survival and growth, inflammatory response and metabolic activity (Cantley, 2002; Laird et al., 2009; Stambolic et al., 1998) (Figure 1.3, left pathway). A key effector of $\mathrm{PI}(3,4,5) \mathrm{P}_{3}$ is the kinase Akt, which is a potent pro-survival signal (reviewed in Datta, Brunet, \& Greenberg, 1999). While Akt itself has numerous targets, it can phosphorylate the GTPase-activating proteins (GAPs) TSC1/2 (Tuberous Sclerosis 1/2), which suppress the GTPase Rheb (Ras homolog enriched in the brain), the major activator of mTOR. In other words, Akt activates mTOR (Inoki, Li, Xu, \& Guan, 2003; Inoki, Li, Zhu, Wu, \& Guan, 2002).

Given that antigen uptake, processing and presentation all require membrane trafficking to lysosomes, that it is unknown how TLR4 signaling may interface with lysosomes, and the recent evidence in the literature that mTOR may play a role in controlling lysosome morphology and dynamics (Krajcovic, Krishna, Akkari, Joyce, \& Overholtzer, 2013; L. Yu et al., 2010), lysosomal membrane trafficking will be introduced.

\subsection{Lysosomal Membrane Trafficking}

Lysosomes are membrane-bound organelles that enclose various hydrolytic enzymes that are optimally active within the acidic lysosome lumen. The low $\mathrm{pH}$ of 4.5-5.5 is maintained by a transmembrane vacuolar $\mathrm{H}^{+}$-ATPase, a multi-subunit complex that actively pumps protons into the organelle. Thus, lysosomes provide an essential niche for molecular digestion while the rest of the cell is protected from 
damage. Important pathways like endocytosis, phagocytosis and autophagy all converge on the lysosome and cells rely on proper lysosomal function for acquisition of nutrients, microbial killing and clearing dead cells, debris and damaged organelles from the system (Figure 1.1) (Gahl, 1989; Hochreiter-Hufford \& Ravichandran, 2013; Mizushima, 2007). The endocytic pathway, which mirrors the phagocytic pathway is described in more detail below.

\subsubsection{Microtubule-Based Motility}

Eukaryotic cells maintain structure, movement and organellar distribution and reorganization through polymerization of filaments that collectively constitute the cell cytoskeleton. Globular actin can polymerize into filaments that are largely found at the cell cortex and aid cell motility, plasma membrane ruffling and shortrange vesicular transport. Other critical processes such as cell division, long-range vesicular transport and maintenance of the general distribution of organelles are achieved by microtubules. Microtubules are generated through the polymerization of $\alpha$ - and $\beta$-tubulin monomers into long protofilaments that in turn assemble into long rigid filaments (tubes) throughout the cytosol. Initial nucleation of a filament occurs at the microtubule organizing center (MTOC), typically juxtaposed at the nucleus. The assembly of tubulin monomers in each protofilament is such that $\alpha$ tubulin is exposed at one end and $\beta$-tubulin is always exposed at the opposite end, providing polarity to the microtubules. The ends are thus designated $(-)$ and $(+)$ where $\alpha$ - or $\beta$-tubulin is exposed, respectively. Microtubule polymerization is significantly more rapid at the (+) end, though microtubule depolymerization, known as catastrophe, also occurs at this end. Certain (+) end-tracking proteins (+TIPs) like CLIP-170 (cytoplasmic inker protein of $170 \mathrm{kDa}$ ) can cap these $(+)$ ends to stabilize them and promote depolymerization rescue. Microtubule stability is alternatively enhanced by post-translational modifications like acetylation.

One of the most notable functions of microtubules is their association with motor proteins that carry out membrane trafficking (Figure 1.4). Microtubule 
motor proteins are a class of ATPases capable of converting chemical energy into mechanical work. Importantly, these molecular 'machines' are able to bind cargo while moving along microtubules. This results in the physical translocation of the cargo, which includes organelles like mitochondria, neuronal vesicles and lysosomes. There are two types of microtubule motor proteins: kinesin and dynein. These motors differ in size and directionality of transport.

The kinesin superfamily (KIFs) consists of 45 genes in mice grouped into 15 families. All kinesins have two things in common: a kinesin motor domain and a coiled-coil domain (Hirokawa, Noda, Tanaka, \& Niwa, 2009). Kinesins bind cargo either directly or through accessory proteins to transport that cargo along microtubules. While the vast majority of kinesins are + end-directed (anterograde) motors, two have been identified as - end-directed (retrograde) motors. Conventional kinesin (kinesin I/KIF5B), initially identified by Vale et al. (Vale, Reese, \& Sheetz, 1985), was later found to be the main + end directed motor driving lysosomal transport.

Retrograde organelle transport is carried out by cytoplasmic dynein (Lin \& Collins, 1992; Lye, Porter, Scholey, \& McIntosh, 1987; Shpetner, Paschal, \& Vallee, 1988). Dynein is a massive motor consisting of two heavy chains, three intermediate chains and four light chains, resulting in a $\sim 1.2$ megadalton complex (Hirokawa, 1998). In addition, dynein interacts with a ten-member protein complex called dynactin, which is important for the function of dynein and has been proposed to serve as a linker between dynein and its cargo through the essential dynactin subunit, dynamitin (Echeverri, Paschal, Vaughan, \& Vallee, 1996). Uncovering the role of dynein has been made possible by the observation that exogenous expression of dynamitin destabilizes the dynactin complex and renders dynein non-functional (Burkhardt, Echeverri, Nilsson, \& Vallee, 1997; Melkonian, Maier, Godfrey, Rodgers, \& Schroer, 2007).

Lysosomal transport is carried out by the coordinated activities of kinesin I and dynein. Retrograde lysosomal transport occurs when dynein-dynactin is recruited by the lysosomal small GTPase Rab7 and its effectors RILP (Rab7 interacting lysosomal protein) and ORP1L (oxysterol-related protein-related 
protein 1L) (Johansson et al., 2007). Rab7 can also bind its effector FYCO1 (FYVE and coiled-coil domain-containing protein 1) to link to kinesin I and carry out anterograde transport of lysosomes. In addition, kinesin I can bind lysosomes through Arl8b, via direct interaction with the Arl8b effector protein SKIP (SifA and kinesin-interacting protein) to carry out anterograde lysosomal transport (RosaFerreira \& Munro, 2011). A description of these small GTPases follows in upcoming sections.

\subsubsection{Organelle Identity and Transport in the Endocytic Pathway}

Organelles carry out specific functions; the mitochondrion generates most of the cells' energy in the form of adenosine triphosphate (ATP), the endoplasmic reticulum (ER) is the site of protein synthesis and endosomes degrade and sort internalized molecules. The process of transporting molecules via membrane fission and fusion events is collectively termed membrane trafficking. Described here is endocytosis, the process by which extracellular milieu and receptors (collectively, cargo) are taken up by invaginations of the plasma membrane into early endosomes that mature and ultimately fuse with lysosomes where cargo is degraded.

During endocytosis, plasma membrane receptors and extracellular molecules are internalized by invaginations of the plasma membrane. This process may or may not be mediated by coat proteins like clathrin. Plasma membrane-derived endocytic cups pinch off to form endocytic vesicles that are trafficked to and fuse with the early endosome, a tubular-vesicular sorting organelle where various receptors are pooled into membrane domains (Abrahamson \& Rodewald, 1981). Membrane extensions fission from the early endosome and are further trafficked to other places within the cell, depending on the cargo they carry. Some vesicles are trafficked back to the plasma membrane via recycling endosomes as is the case with vesicles carrying Low Density Lipoprotein Receptor (LDLR) which delivers the 
nutrient cholesterol into the cell (Anderson, Brown, Beisiegel, \& Goldstein, 1982). Other cargo at the early endosome is siphoned off for degradation.

Cargo destined for degradation is typically ubiquitinated, the process in which ubiquitin proteins are ligated onto the cytosolic portion of cargo molecules carried by early endosomes. This ubiquitin tag recruits proteins of ESCRT (endosomal sorting complexes required for transport) machinery which, through membrane deformation, further compartmentalizes cargo into small intraluminal vesicles to form multivesicular bodies (Miller, Beardmore, Kanety, Schlessinger \& Hopkins, 1986; Katzman, Babst \& Emr, 2001). This ensures that cargo destined for degradation is quarantined for this fate. As early endosomal markers are shed, endosomes further mature by acquiring late endosomal identity markers (Rink, Ghigo, Kalaidzidis, \& Zerial, 2005). Late endosomes also undergo maturation by fusing with lysosomes, the terminal organelle in the endocytic pathway.

Despite constant membrane mixing during intracellular trafficking, cells are able to maintain distinct organelle identities. Cells utilize a multitude of molecules as organelle identity markers and orchestrators of intracellular membrane trafficking, most notably phosphoinositide lipid species and small GTPases.

\subsubsection{Phosphoinositides}

Phosphatidylinositol (PI), which represents no more than $15 \%$ of total cellular phospholipids, can be phosphorylated at any of three positions on its inositol head group, giving rise to seven distinct species of phosphoinositides (PIPs). The six phosphorylated derivatives of PI are each approximately one order of magnitude lower in abundance than PI. Remarkably, despite their low abundance, PIPs control functions as diverse and essential as signaling from receptor tyrosine kinases to membrane trafficking. The synthesis and turnover from one PIP to another relies on the spatiotemporal localization and action of various lipid kinases and lipid phosphatases. This generates a unique subcellular distribution of PIPs. Both 
differential distribution and rapid interconversion render these phospholipids ideal markers of membrane identity and mediators of signaling.

Various protein modules exist which recognize phosphoinositides. As previously discussed for example, $\mathrm{PI}(3,4,5) \mathrm{P}_{3}$ which is transiently generated by $\mathrm{PI} 3 \mathrm{~K}$ from $\mathrm{PI}(4,5) \mathrm{P}_{2}$, is recognized by the plekstrin homology $(\mathrm{PH})$ domain of the signaling kinase Akt. The recruitment of Akt to the membrane via this PH domain$\mathrm{PI}(3,4,5) \mathrm{P}_{3}$ interaction is critical for the activation of Akt and the propagation of a signaling cascade that controls cell growth. In the endocytic pathway, $\operatorname{PI}(3) \mathrm{P}$ is generated on the early endosomal membrane and is recognized by the FYVE (Fab1, YOTB, Vac1, EEA1) domain of PIKfyve, a lipid kinase that converts PI(3)P into $\mathrm{PI}(3,5) \mathrm{P}_{2}$, a lipid which is more characteristic of late endosomes (Gary, Wurmser, Bonangelino, Weisman, \& Emr, 1998; Hurley \& Meyer, 2001; Ikonomov, Sbrissa, \& Shisheva, 2001). Thus, the lipids themselves as well as their various effector proteins all contribute to establishing organelle identity.

\subsubsection{The Small GTPases and Effector Proteins}

In addition to phosphoinositides, organelle identity and membrane trafficking is established by members of the Ras superfamily of monomeric guanosine triphosphatases (monomeric or small GTPases). Small GTPases convert from an "active" GTP-bound form to an "inactive" GDP-bound form (Figure 1.5). All small GTPases contain conserved regions called switch I and switch II, which experience conformational alterations upon GDP/GTP exchange. When GDP is bound, the switch I and switch II regions are relaxed and the GTPase exists in a conformation that is unable to bind effector proteins. In order for the GTPase to be switched "on", guanine nucleotide exchange factors (GEFs), that promote the switching of GDP for GTP, are required. GEFs act by binding GTPases and inserting residues within the GDP-GTPase interface and destabilizing the interaction. This ultimately results in loss of GDP, followed by rapid subsequent acquisition of GTP. When GTP is bound, critical conserved residues in switch I and switch II interact directly with the $\gamma$ - 
phosphate of GTP to keep the GTPase in a conformation that is favourable for effector binding. As their name suggests, the small GTPases are enzymes that have an intrinsic ability to hydrolyze GTP into GDP. However, this process is extremely slow and inefficient. GTPase activating proteins (GAPs) help induce the hydrolysis of GTP by GTPases by binding the GTPase and stabilizing the transition state, and possibly contributing residues for the GTP hydrolysis in the process (Scheffzek et al., 1997).

Rab and Arf GTPases are heavily involved in vesicular transport and different Rabs and Arfs are specific to different membrane-bound organelles (reviewed in Chavrier \& Goud, 1999). When GTP-bound, they are localized to the cytosolic face of organelle membranes and recruit effector proteins (Grosshans, Ortiz, \& Novick, 2006). The plethora of effectors that bind various GTPases is diverse, and effector proteins have many different functions in cells including membrane tethering, fusion and transport. When GDP-bound, these GTPases detach from membranes and become cytosolic. Importantly, they are unable to bind effectors while in this inactive state. This ability of GTPases to act as 'molecular switches' is critical for membrane trafficking and for bestowing identity to the compartments they bind.

\subsection{Rab7 and Arl8b}

Rab7 and the Arf-like GTPase Arl8b are involved in late endosomal/lysosomal traffic (Bagshaw, Callahan, \& Mahuran, 2006; Nakae et al., 2010; Wang, Ming, Xiaochun, \& Hong, 2011). When GTP-bound, they localize to the lysosomal membrane and recruit effector proteins that are known to control lysosomal dynamics. The activity of Rab7 and Arl8b, like other GTPases, is dependent on GEFs and GAPs. A welldefined Rab7 GEF is the dimer Mon1-Ccz1, initially discovered as the major GEF for the yeast Rab7 homolog, Ypt7 (Nordmann et al., 2010; Poteryaev, Fares, Bowerman, \& Spang, 2007). In addition, the homotypic fusion and protein sorting complex (HOPS) which mediates late endosome/lysosome tethering and fusion has GEF activity toward Rab7 via its Vps39 subunit (Wurmser, Sato, \& Emr, 2000). There are 
also two known GAPs of Rab7, the TBC (Tre2/Bub2/Cdc16) domain-containing proteins TBC1D15 and Armus (TBC1D2A) (Peralta, Martin, \& Edinger, 2010; Frasa et al., 2010). Unlike Rab7, much less is known about the regulation of Arl8b. As yet, no Arl8b GEFs or GAPs have been identified, though a candidate GEF may be the lysosomal multi-subunit complex BORC (BLOC one-related complex), which directly interacts with Arl8b and promotes Arl8b-dependent lysosomal transport (Pu et al., 2015). Although Pu et al. failed to detect GEF activity of recombinant BORC in vitro, the authors report the possibility that post-translational modifications absent in the recombinant complex may be necessary for its activity.

As described, small GTPases exert functions via their effector proteins. Several Rab7 and Arl8b effector proteins have been identified as key players that help carry out late endosomal/lysosomal traffic, including RILP and FYCO1. RILP has been shown to link Rab7 with the minus-end-directed microtubule motor dynein (Jordens et al., 2001). This occurs as another Rab7 effector, ORP1L (oxysterol-binding protein-related protein 1L), is recruited to Rab7-RILP to facilitate the linkage of late endsosomes/lysosomes to dynactin, a regulatory subunit of dynein (Johansson et al., 2007). Conversely, the Rab7 effector FYCO1 has been implicated as an adapter of the plus-end-directed microtubule motor kinesin I (Jordens et al., 2001; Pankiv et al., 2010; Wang, Ming, Xiaochun, \& Hong, 2011). By linking late endosome/lysosome-bound Rab7 to microtubule motor machinery, these effectors are key regulators of the trafficking of these organelles. The Arl8b effector protein SKIP, was identified as the link between lysosomes and kinesin-1, by binding directly to active Arl8b and the kinesin I light chain (Boucrot, Henry, Borg, Gorvel, \& Meresse, 2005; Rosa-Ferreira \& Munro, 2011). Indeed, cells silenced for SKIP displayed an accumulation of lysosomes in the perinuclear region, confirming its critical role in peripheral lysosome distribution (Rosa-Ferreira \& Munro, 2011). In these ways, Rab7 and Arl8b promote minus and plus end-directed lysosomal transport (Jordens et al., 2001; Pankiv et al., 2010, Bagshaw, Callahan, \& Mahuran, 2006).

While kinesin I and dynein are required for lysosomal transport, regulators of lysosomal morphology and trafficking continue to be uncovered. Signals that 
cause increased GTP loading and membrane association of the small GTPases Rab7 and Arl8b in turn determine motor protein recruitment and lysosomal morphology/transport. Many of the regulators of lysosomal morphology and trafficking remain elusive. This thesis provides tantalizing evidence that the Ser/Thr kinase mTOR (mechanistic Target of Rapamycin) may be one such important regulator.

\subsection{Mechanistic Target of Rapamycin and Lysosomes}

Cells respond to nutrient status and integrate signaling for growth through the protein kinase mTOR, which, not surprisingly, is deregulated in cancer and other diseases (Dazert \& Hall, 2011; Zoncu, Sabatini, \& Efeyan, 2011). mTOR itself is the catalytic subunit of at least two known complexes, mTOR complex 1 (mTORC1) and mTORC2 (described in yeast by Loewith et al., 2002). mTOR is a $\sim 280 \mathrm{kDa}$ protein that bears a C-terminal kinase domain that highly resembles the catalytic domain of PI3K (reviewed in Wullschleger, Loewith, \& Hall, 2006). Thus, mTOR is regarded as a PI3K-related kinase.

mTOR exerts its effects on cell growth and proliferation mainly via its substrates which generally control protein translation. Remarkably few mTOR substrates are known, and the two most well characterized are the translational repressor 4E-BP1 (eukaryotic initiation factor 4E-binding protein 1) and the ribosomal protein S6K (S6 kinase) (Chung, Kuo, Crabtree, \& Blenis, 1992; von Manteuffel, Gingras, Ming, Sonenberg, \& Thomas, 1996). As the name suggests, 4EBP1 binds and suppresses the eukaryotic initiation factor 4E (eIF-4E) which otherwise promotes protein synthesis by binding to mRNA 5 '-cap to recruit protein translation machinery. Upon mTOR stimulation, 4E-BP1 is phosphorylated, preventing binding to eIF-4E and liberating it for protein translation (Pause et al., 1994; von Manteuffel, Gingras, Ming, Sonenberg, \& Thomas, 1996). On the other hand, S6K, when phosphorylated and activated by mTOR, is capable of 
phosphorylating its own substrate, the ribosomal protein S6, as well as other components of translational machinery collectively enhancing protein synthesis.

One of the most important functions of mTOR is its ability to suppress autophagy. Autophagy is a homeostatic and physiologically important process by which cells degrade cytosolic components, including damaged organelles and protein aggregates, by sequestering that cargo into a double-membrane structure called a phagophore, or isolation membrane (Stromhaug, Berg, Fengsrud, Seglen, 1998; Lamb, Yoshimori, \& Tooze, 2013; Tooze \& Yoshimori, 2010). The isolation membrane, marked by the presence of the ubiquitin-like protein LC3 (light chain 3), extends around the cargo and fuses with itself to form an organelle called the autophagosome, which further fuses with lysosomes to form autophagolysosomes. This fusion with lysosomes is critical for the downstream degradation of autophagosomal cargo by lysosomal hydrolases. Under nutrient-rich conditions, mTOR is active and directly suppresses the formation of autophagosomes, by phosphorylating and inhibiting the activity of ULK1/2 (Unc51-like autophagy activating kinase 1) and Atg13 (Autophagy-related protein 13), core machinery of autophagosome biogenesis (Jung et al., 2009; Jung, Ro, Cao, Otto, \& Kim, 2010). Autophagy becomes activated upon nutrient starvation and/or mTOR inhibition.

The two mTOR complexes, mTORC1 and mTORC2, share some subunits including deptor (DEP domain-containing mTOR-interacting protein), mLST8 (mammalian lethal with SEC13 8), Tti1 (tel2-interacting protein 1), Tel2 (telomere length regulation protein 2) (Laplante \& Sabatini, 2013). However, mTORC1 contains the subunits Raptor (regulatory associated protein of mTOR) and PRAS40 (proline-rich Akt1 substrate) whereas mTORC2 contains Rictor (rapamycin insensitive component of TOR), mSin1 (stress-activated MAP-kinase interacting protein 1) and protor1/2 (protein observed with rictor) (Laplante \& Sabatini, 2013). Unlike mTORC1, mTORC2 is not inhibited by rapamycin, the macrocyclic antifungal metabolite produced by the soil microorganism Streptomyces hygroscopicus, found to target and inhibit mTORC1, hence the name of the kinase itself. In the last few years however, research has introduced many new compounds that are potent and 
specific mTOR inhibitors including torin1, pp242 and wye687 (Feldman et al., 2009; Liu et al., 2010; K. Yu et al., 2009).

The two different complexes also have different functions in cells. For example, mTORC1 which is localized to lysosomes, has been shown to integrate signals from nutrients like amino acids and growth factors, as well as energy and stress (Zoncu, Sabatini, \& Efeyan, 2011). The lysosomal localization of mTORC1 is consistent with the localization of its activator, the lysosomal small GTPase Rheb (Ras homolog enriched in the brain) (Inoki, Li, Xu, \& Guan, 2003). Surprisingly, much less is known about mTORC2 except that insulin activates Akt in an mTORC2dependent manner (Sarbassov, Guertin, Ali, \& Sabatini, 2005), thus providing the notion that mTORC2 likely integrates growth factor signaling. Outputs of mTORC2 include cell survival and cytoskeletal organization (Laplante \& Sabatini, 2013).

Interestingly, it is now appreciated that lysosomes can sense stress and the metabolic state of the cell through mTOR (Sengupta, Peterson, \& Sabatini, 2010; Settembre et al., 2012; Settembre, Fraldi, Medina, \& Ballabio, 2013). This ability of mTOR to sense nutrients via lysosomal association, has gained much interest in recent years and is briefly introduced below.

\subsubsection{Nutrient Sensing at the Lysosome}

It was previously observed that mTORC1 re-localized from a predominantly cytosolic pool to lysosomes upon addition of amino acids (Sancak et al., 2008). This

prompted the investigation of the mechanisms by which amino acids promote the localization of mTOR to the lysosome where it is activated. It has since been proposed that amino acids signal from within lysosomes by interacting with the lysosomal $\mathrm{H}^{+}$V-ATPase, which in turn activates the multi-protein complex Ragulator. Ragulator is a GEF for Rag GTPases that recruit mTOR to the lysosomal membrane, bringing it in close proximity to its activator, the small GTPase Rheb (Figure 1.6). 
In addition to being associated with lysosomes and bestowing these nutrient sensing properties to the organelle, mTOR has been shown to play a role in regulating lysosomal morphology as well.

\subsubsection{Regulation of Lysosomal Dynamics by mTOR}

Not only is mTOR localized to lysosomes as part of mTORC1, it is required for autophagic lysosome reformation, a process in which lysosomes are retrieved through tubular intermediates from autophagolysosomes at the end of autophagy (L. Yu et al., 2010). During starvation, cells generate autophagosomes, intracellular membrane-bound organelles that sequester cytosolic components including other organelles, and target these components to lysosomes for degradation. (Figure 1.1D) The fusion with lysosomes to form autophagolysosomes allows for breakdown of sequestered material into smaller precursor molecules, which can be retrieved from lysosomes via egress through lysosomal channels and transporters. This process effectively replenishes nutrients, and cells can survive longer during starvation periods. During this nutrient recovery period, lysosomes, which were previously fused with autophagosomes to generate autophagolysosomes, begin to re-form and the total cellular lysosomal pool begins to appear as it did prior to starvation. This process of "autophagic lysosome reformation" (ALR) is a method of homeostasis and naturally occurs through tubular intermediates. That is, lysosomes begin to bud off of autophagolysosomes in long tubular structures, which ultimately pinch off to form bona fide lysosomes. Interestingly, mTOR was found to be required for this process, as inhibition with rapamycin prevented the reformation of lysosomes following autophagy and blocked formation of tubular lysosomal intermediates (L. Yu et al., 2010).

In addition, mTOR has also been reported to regulate fission from phagosomes and entotic vacuoles, cell-in-cell structures that result from the cell death mechanism, entosis (Krajcovic et al., 2013). Phagosomes and entotic vacuoles mature by fusing with lysosomes where their cargo is degraded, followed by 
subsequent fission steps during late-stage maturation. Krajcovic et al. found that mTOR gene silencing or inhibition by torin 1 blocked this late-stage fissioning step from both entotic vacuoles and phagosomes.

As yet, it is unclear what the substrates of mTOR might be that are involved in regulation of organelle morphology, as the only substrates known to date are found to function in protein translation. Given the lysosomal localization of mTOR, and its implications in regulating ALR and phagosome/vacuole membrane dynamics, this kinase is poised to be a master regulator of lysosomal morphology.

\subsection{Lysosome Tubulation}

Lysosomes are normally small punctate organelles, appearing as dots dispersed throughout the cell cytosol. In activated innate immune cells however, lysosomes become strikingly tubular in morphology. Joel Swanson and colleagues observed highly tubular lysosomes (TLS) upon activation of macrophages with phorbol 12myristate 13-acetate (PMA) (Swanson, Bushnell, \& Silverstein, 1987), a potent activator of protein kinase $\mathrm{C}(\mathrm{PKC})$ which is a key intermediate in the $\mathrm{NF \kappa B}$ signaling pathway. In addition, the dendritic cell MIIC, a lysosome-related organelle, undergoes the same profound morphological changes to form tubular MIIC (tMIIC) when cells are exposed to the MAMP LPS (Chow, Toomre, Garrett, \& Mellman, 2002). Meanwhile, it was reported in the laboratory of Hidde Ploegh that engagement of DCs with T-cells was also sufficient to induce the tubular reorganization of the lysosome-related MIIC.

While the phenotype of TLs is striking, their function remains largely elusive. There is evidence that tubulation of lysosomes can enhance retention of fluid phase, that is, soluble molecules taken up by stimulated pinocytosis are routed to the tubular lysosomal network and retained (Swanson, Yirinec, \& Silverstein, 1985;

Swanson, Burke, \& Silverstein, 1987). Furthermore, tubulation is thought to aid phagosome maturation by enhancing the fusion of phagosomes with lysosomes (Harrison, Bucci, Vieira, Schroer, \& Grinstein, 2003). Harrison et al observed tubular 
membrane extensions emanating from phagosomes and fusing with late endosomes/lysosomes. Finally, and possibly most convincing, is the role of lysosome tubulation in antigen presentation. Stimulation of DCs with LPS resulted in a radial extension of tMIIC emanating from MIIC clusters near the nucleus toward the cell periphery (Chow, Toomre, Garrett, \& Mellman, 2002). Interestingly, DCs relocalized their MHCII from intracellular MIIC to the plasma membrane within hours of LPS treatment (Chow, Toomre, Garrett, \& Mellman, 2002). Similarly, engagement of DCs with T-cells, which induces secretion of MHCII to the immunological synapse for antigen presentation, proceeded with a rapid rearrangement of the MIIC into long tubules which were polarized toward the T-cell/DC synapse (Bertho et al., 2003; Boes et al., 2002, 2003).

How do TLs form? Interestingly, though there are several studies documenting their formation in activated macrophages and DCs, little is known about how tubular lysosomes are generated in cells. Early works by Swanson et al. identified a role for microtubules and kinesin in tubular lysosome biogenesis in macrophages (Hollenbeck \& Swanson, 1990; Swanson, Locke, Ansel, \& Hollenbeck, 1992). It was fifteen years later that Vyas et al. described the requirement for the TLR adaptor MyD88 in LPS-induced TL biogenesis in DCs and characterized the tubules further as being positive for the lysosomal markers CD63 and LAMP1 (lysosome associated membrane protein 1) (Vyas et al., 2007). As stated previously, mTOR has also been implicated in regulating lysosomal morphology, including tubulation, though in a different context. Clearly, understanding how lysosome/MIIC tubulation is regulated is critical to understanding their seemingly important functional roles. 


\subsection{Project Goals and Rationale}

Lysosomes are vital organelles, regulating diverse processes including turnover of cytoplasmic components via autophagy, cholesterol homeostasis via the lysosomal protein Niemann-Pick C1 (NPC1) and plasma membrane repair via focal exocytosis to name a few. In an immunological context, lysosomes and lysosome-related organelles play critical roles. For example, in macrophages, lysosomes fuse with phagosomes to digest microbes and protect the host. In professional APCs like DCs, the lysosome-related MIIC processes and delivers antigen to the plasma membrane for T-cell recognition (Neefjes, Stollorz, Peters, Geuze, \& Ploegh, 1990; Neefjes, 1999). And in cytotoxic lymphocytes, lytic granules are secreted to kill virallyinfected target cells (Burkhardt, Hester, Lapham, \& Argon, 1990; Peters et al., 1991). With this broad array of important functions in organisms, understanding how the morphology and identity of lysosomes is regulated is of great interest.

Lysosome tubulation, which is a striking morphological change that these organelles undergo, remains elusive, yet may be essential to the proper functioning of innate immune cells, and potentially other cells as well. Thus, for my Ph.D. thesis, I set out to explore how innate immune cells form tubular lysosomes. I hypothesize that lysosome tubulation is driven by the coordinated actions of lysosomal small GTPases Rab7 and Arl8b and their effectors that link to microtubule-based motor proteins to help stretch lysosomes into tubules. Furthermore, I hypothesize that mTOR plays a role in lysosome tubulation by integrating LPS signaling to activate lysosome tubulation machinery. To address these hypotheses, the following goals were set:

1) Develop a model system to study tubular lysosome biogenesis

2) Determine the molecular machinery that drives tubulation of lysosomes

3) Elucidate the signaling pathway that leads to lysosome tubulation

4) Identify the role of mTOR in lysosome tubulation 
Figures for Chapter 1 
Figure 1.1: Lysosomal Trafficking Pathways. (A) All cells undergo endocytosis, the mechanism of constitutive uptake of cargo including plasma membrane receptors, receptor-bound cargo and fluid-phase molecules. Cargo is internalized via invagination of the plasma membrane that pinches off to form an endocytic vesicle. The lumen of the endocytic vesicle resembles the extracellular milieu. Endocytic vesicles fuse with early endosomes, a tubule-vesicular sorting station. The diagram depicts some cargo receptors being sorted within tubules (blue receptor) that will pinch off and be delivered elsewhere, such as recycled back to the plasma membrane. Other cargo destined for degradation (red receptor) and may be further compartmentalized within intraluminal vesicles as the endosome becomes a multivesicular body. This compartment matures by acquiring markers of late endosomes and progressively acquires a lower-pH lumen. Ultimately, cargo destined for degradation is trafficked to the lysosome, the most acidic and degradative organelle in the cell. Here, cargo molecules are degraded into building blocks by the actions of multiple acid hydrolases. (B) Leukocytes like macrophages and DCs are professional phagocytes and therefore undergo phagocytosis. During this receptormediated process, binding of bacteria by leukocyte phagocytic receptors (ie. Fc $\gamma$ receptors bind IgG on surfaces of bacteria) triggers remodeling of the cytoskeleton to form actin-driven pseudopodia that extend and surround the bacterium. Once internalized, the phagosome matures by subsequent fusion steps between early and late endosomal compatments. Ultimately, phagosomes must fuse with lysosomes to ensure bacterial killing. (C) Leukocytes like macrophages and particularly immature DCs are particularly good at macropinocytosis. This actin-driven process is non-specific and proceeds by the extension of large plasma membrane ruffles into the extracellular space which collapse and fuse with the plasma membrane culminating in the internalization of a large amount of extracellular fluid. In this way, APCs like macrophages and DCs are able to continuously monitor their extracellular environment for soluble antigens. (D) Autophagy is a pathway important for homeostasis that all cells undergo, which also converges on the lysosome. During this process, damaged organelles, protein aggregates and other cytosolic components, are surrounded by a forming double membrane that extends 
and seals to form an organelle called the autophagosome. Autophagosomes ultimately must fuse with lysosomes where the inner membrane and sequestered components within are degraded into precursor molecules. This evolutionarily conserved pathway is important for cell survival during starvation and stress.

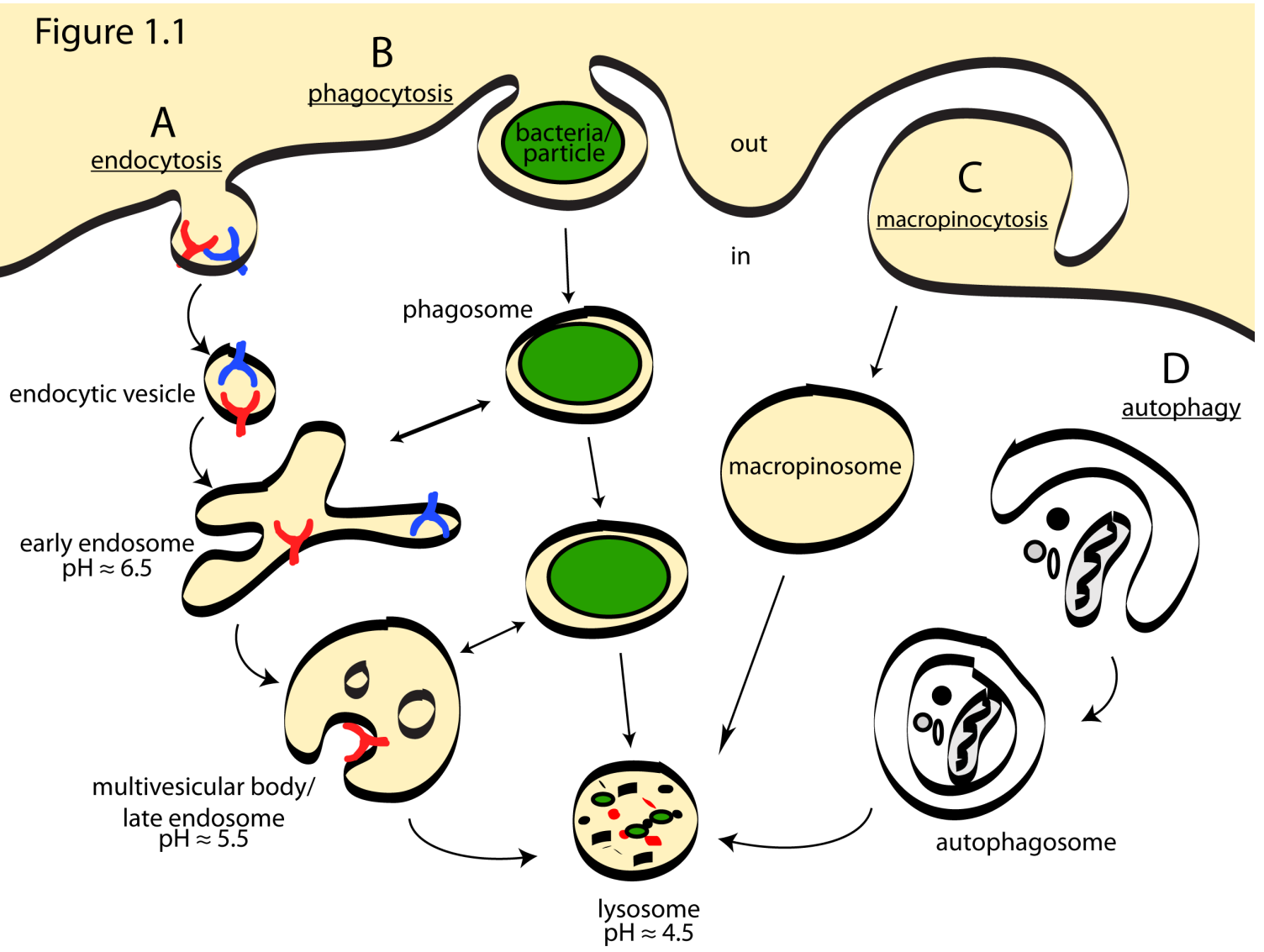


Figure 1.2: Antigen Processing and Presentation by MHCII in Antigen Presenting Cells (APCs). Bacteria and other antigenic sources are taken up by the APC and trafficked to lysosomes where they encounter lysosomal proteases (Cathepsins) that degrade the cargo into smaller peptides, antigens. Meanwhile, MHCII molecules are synthesized in the endoplasmic reticulum along with invariant chain, an endogenous protein that binds and blocks the antigen-binding groove of MHCII, preventing premature antigen loading by self peptides. The MHCII-invariant chain complex is trafficked through the biosynthetic/secretory pathway directly to late endosomes and lysosomes. Within lysosomes, Cathepsin S degrades most of the invariant chain leaving behind a small peptide, CLIP (Class II associated invariant chain peptide), within the antigen-binding groove. In the lysosome, where antigen is encountered, MHCII-CLIP binds a chaperone-like molecule (not depicted) called HLA-DM (human leukocyte antigen-DM) that facilitates the release of CLIP allowing higher-affinity antigen to bind within the groove. The antigen-MHCII complex is transported to the plasma membrane where they bind and activate CD4+ $\mathrm{T}$-cells via the TCR (T-cell receptor) and other co-receptors not depicted, including CD4. 
Figure 1.2

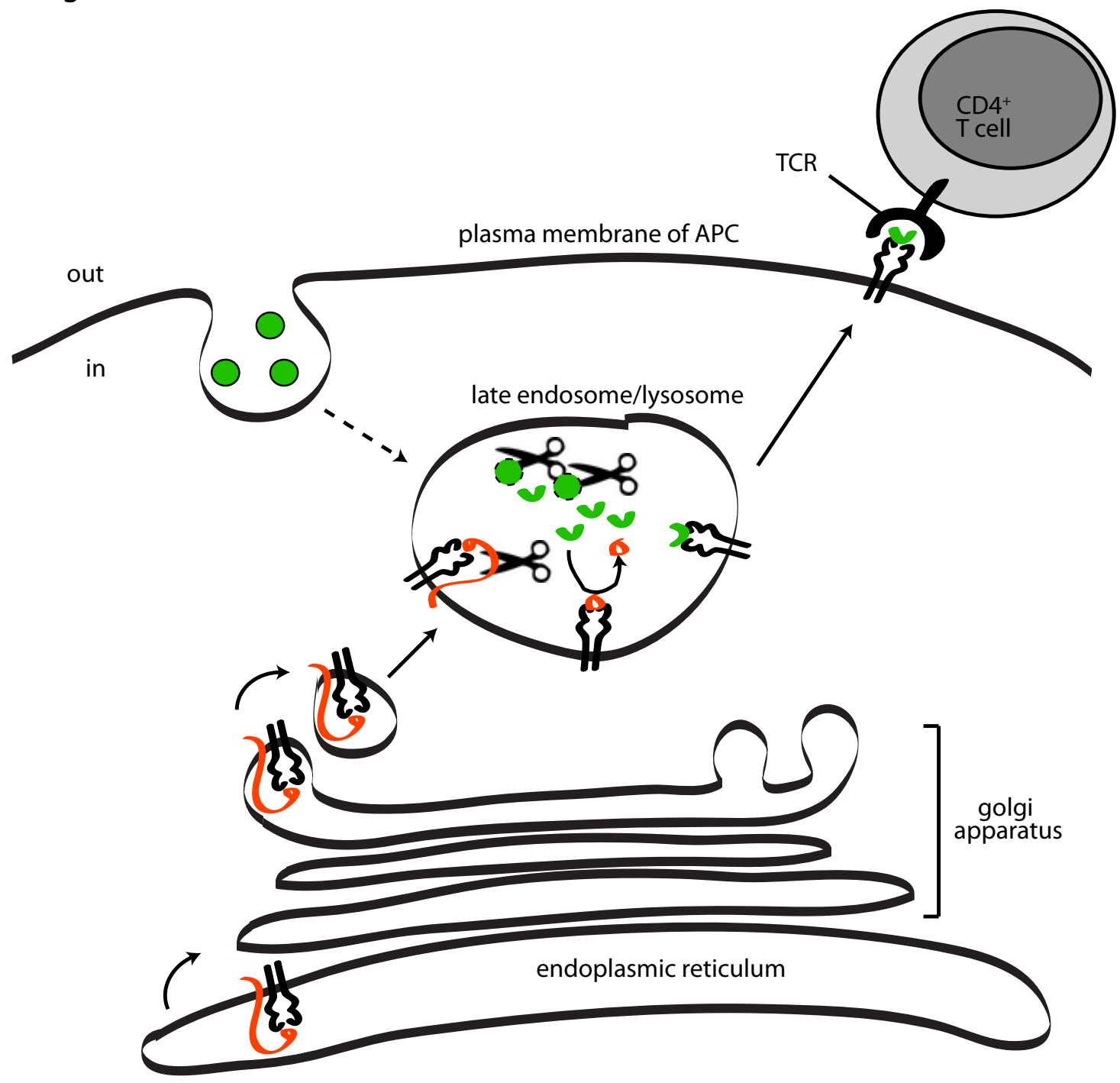

$\begin{array}{|lll|}\left\{\begin{array}{l}\text { MHCII } \\ \text { invariant chain }\end{array}\right. & \text { OLIP } & \text { antigenic source (ie. bacterium) } \\ & \text { lysosomal proteases } & \text { antigen }\end{array}$


Figure 1.3: TLR4 Signaling Pathways. Innate immune cells respond to LPS through the TLR4 (Toll-like receptor 4). TLR4 activation can trigger multiple TLR adaptor proteins. Signaling can proceed via the adaptor protein TRIF (TIR-domain containing adaptor inducing interferon- $\beta$ ) to activate the kinases TBK1 (TANKbinding kinase 1) and IKK (IkB kinase), which in turn phosphorylate IRF3 (Interferon regulatory factor 3 ), a transcription factor that controls production of type I interferons. Another major TLR4 adaptor is MyD88 (Myeloid differentiation primary response protein 88) that can recruit IRAK1 (IL-1 receptor associated kinase 1) and IRAK4. This in turn activates TRAF6 (TNF receptor-associated factor 6) ultimately causing degradation of IkB (inhibitor of NFkB) and nuclear translocation of NFkB (nuclear factor kappa B) that controls transcription of proinflammatory cytokines. Finally, MyD88 can stimulate PI3K (phosphatidylinositol 3kinase), which generates the signaling phospholipid $\mathrm{PI}(3,4,5) \mathrm{P}_{3}$ at the plasma membrane (not depicted). This in turn recruits the PH domain-containing protein Akt leading to its activation (not depicted). Akt can activate mTOR (mechanistic target of rapamycin) via phosphorylation and suppression of TSC1/2 (Tuberous sclerosis $1 / 2$ ), a Rheb GAP, which effectively enhances the activity of the mTOR activator Rheb (dashed line). mTOR in turn regulates cell growth by inducing protein translation, inhibiting autophagy and generally promoting anabolic processes. 
Figure 1.3

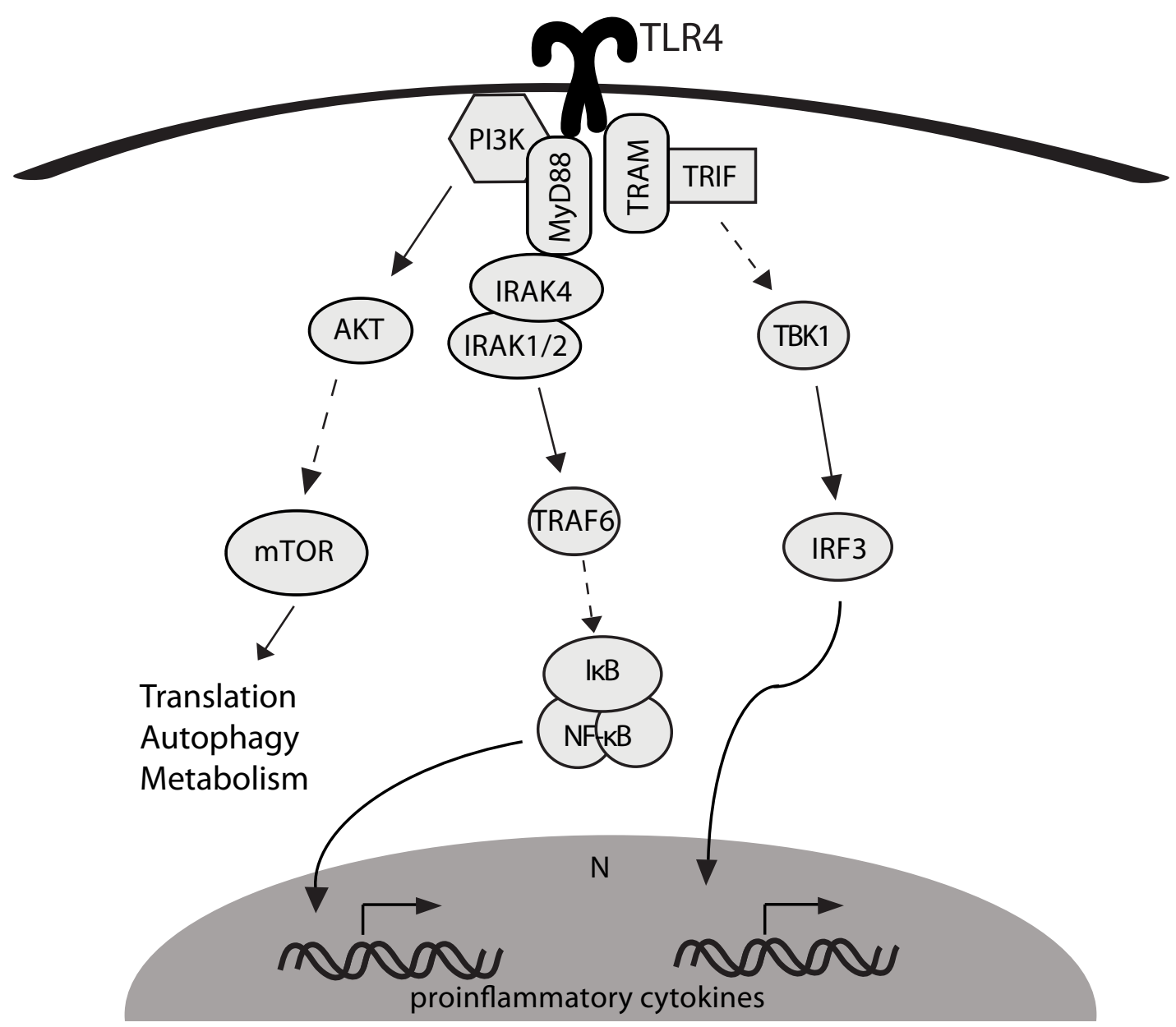


Figure 1.4: Microtubule-Based Organelle Transport. This schematic depicts roughly how organelle transport is accomplished in leukocytes. Microtubules polymerize from the MTOC, near the nucleus (-) outward toward the cell periphery $(+)$ and provide a scaffold along which organelle transport takes place. Large cytosolic proteins, kinesins, and the massive complex dynein, "walk" along microtubules in the + end-directed and - end-directed directions, respectively. Interestingly, both motor proteins are ATPases that convert chemical energy into mechanical work by hydrolyzing ATP. This results in small conformational changes that ultimately generate "power strokes" to move along microtubules. In addition, these motors harbour cargo-binding domains, which are the regions that directly interact with cargo directly, or in the case of organelles, an effector protein that is linked to the organelle. For example, lysosomes link to kinesin through the small GTPase Arl8b and its effector SKIP. (Image adapted from Vale, 2003) 
Figure 1.4

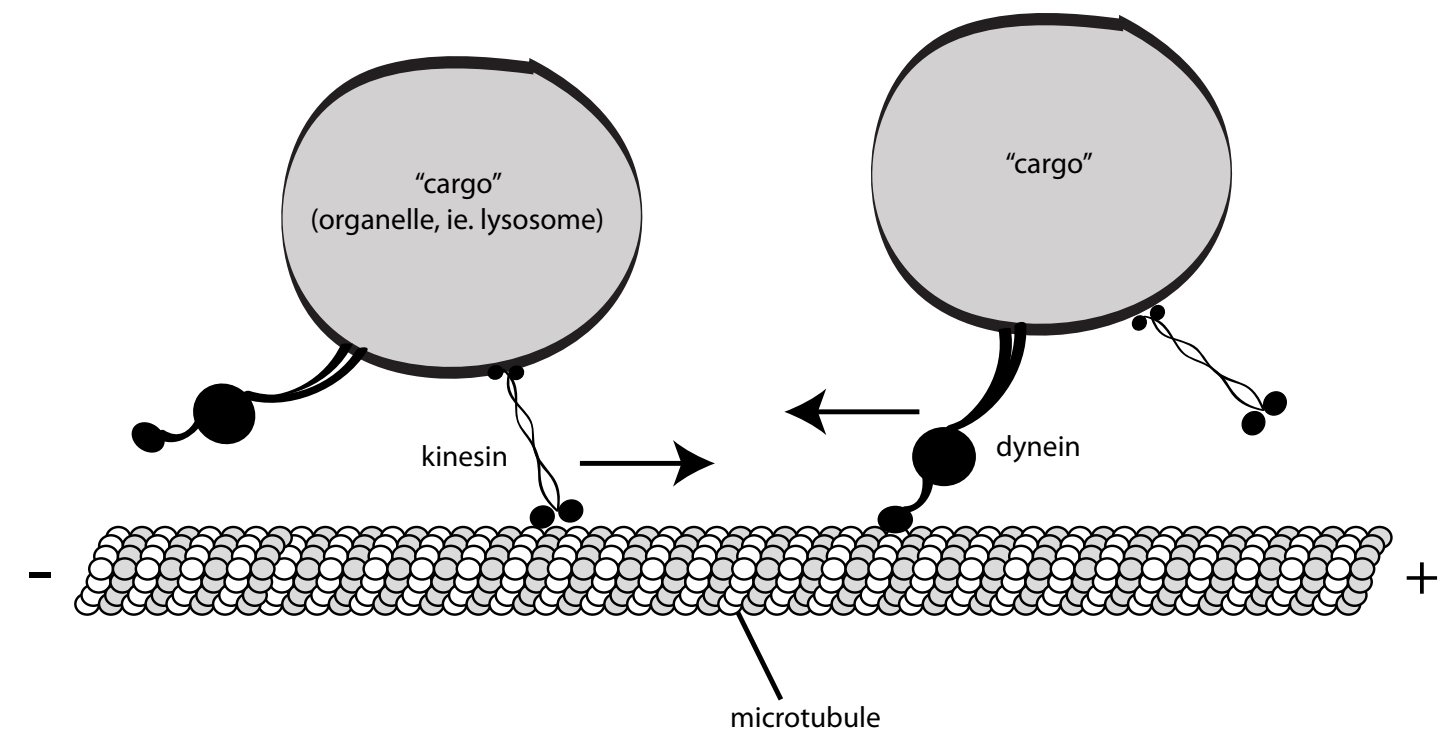


Figure 1.5: Activation Cycle of Small GTPases. Small GTPases can cycle from an active "on" state to an inactive "off" state. This is determined by the nucleotide bound to the GTPase. Small GTPases that are GTP-bound are in an "on" state, meaning that they are in a conformation capable of recruiting effector molecules, and in the case of the lysosomal small GTPases Rab7 and Arl8b, the "on" state exposes a lipid modification which targets the GTPase to the membrane of the organelle. The effectors that GTPases bind in the "on" state dictate the outcome for the cell. For example, effector proteins can be long tethering molecules that bring organelles in close proximity to facilitate membrane fusion. Switching a GTPase "on" requires a GEF (guanine nucleotide exchange factor) that facilitates the dissociation of GDP from the GTPase, at which point the GTPase quickly acquires GTP and becomes activated. Conversely, switching to an "off" state means that the GTPase is bound to GDP instead of GTP. This is accomplished by the intrinsic enzymatic activity of the GTPase that hydrolyzes the terminal phosphate group from GTP, converting it to GDP and releasing inorganic phosphate in the process. This intrinsic enzymatic activity is slow and made efficient in the presence of a GAP (GTPase activating protein). Importantly, when GDP bound and "off" the GTPase exists in a conformation that is unable to bind effector proteins. 
Figure 1.5

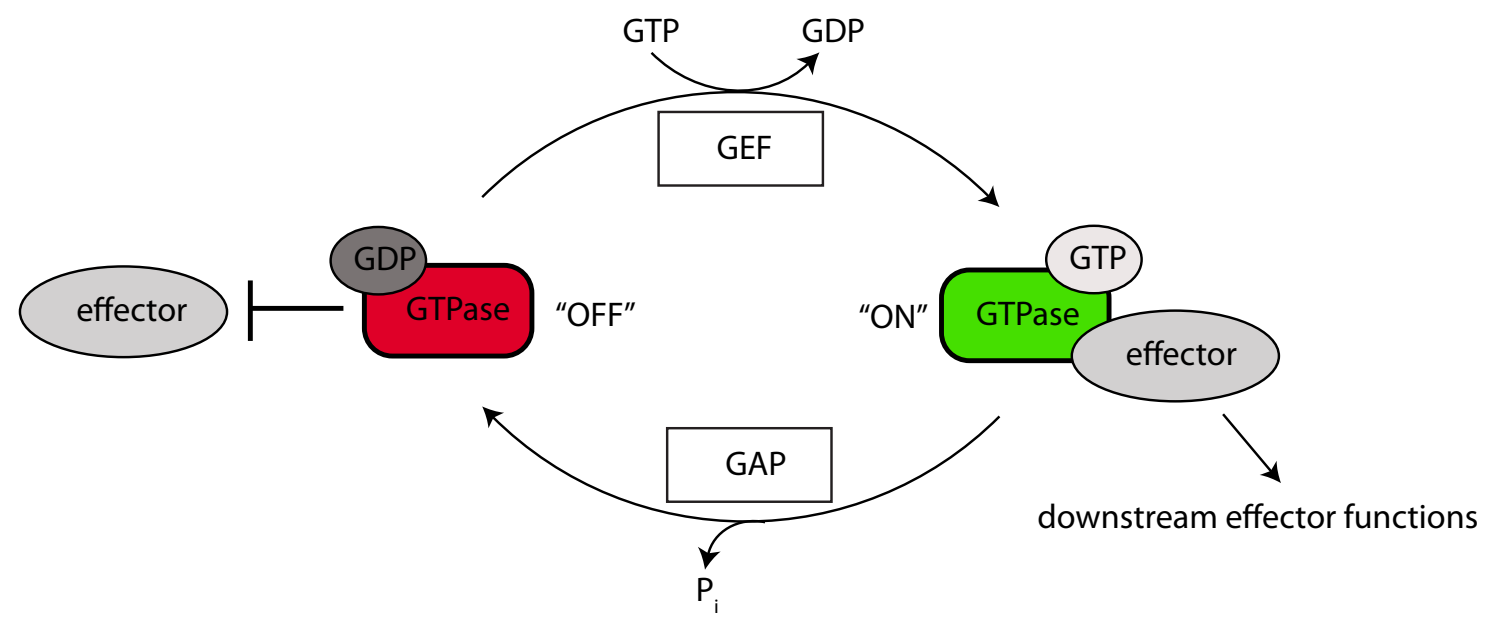


Figure 1.6: Lysosomal Inside-Out Activation of mTORC1 by Amino Acids. Amino acids that are internalized via endocytosis, or transported from the cytosol across the lysosomal membrane into the lysosomal lumen, interact directly with V-ATPase from within lysosomes. This "sensing" by the V-ATPase is thought to convey information to Ragulator, a pentameric complex that is tethered to the lysosome on the cytosolic face. In turn, Ragulator, which has GEF activity toward lysosomal Rag GTPases, activates Rags which recruit and directly bind to mTORC1. This is considered to be important for physically bringing mTOR into close proximity to its activator, the lysosomal small GTPase Rheb. This translocation of mTORC1 to the lysosomal membrane via Rags results in the activation of mTOR at this site. Thus, inside-out amino acid sensing by lysosomes can activate mTOR. (Image modified from Bar-Peled, Schweitzer, Zoncu, \& Sabatini, 2012) 
Figure 1.6

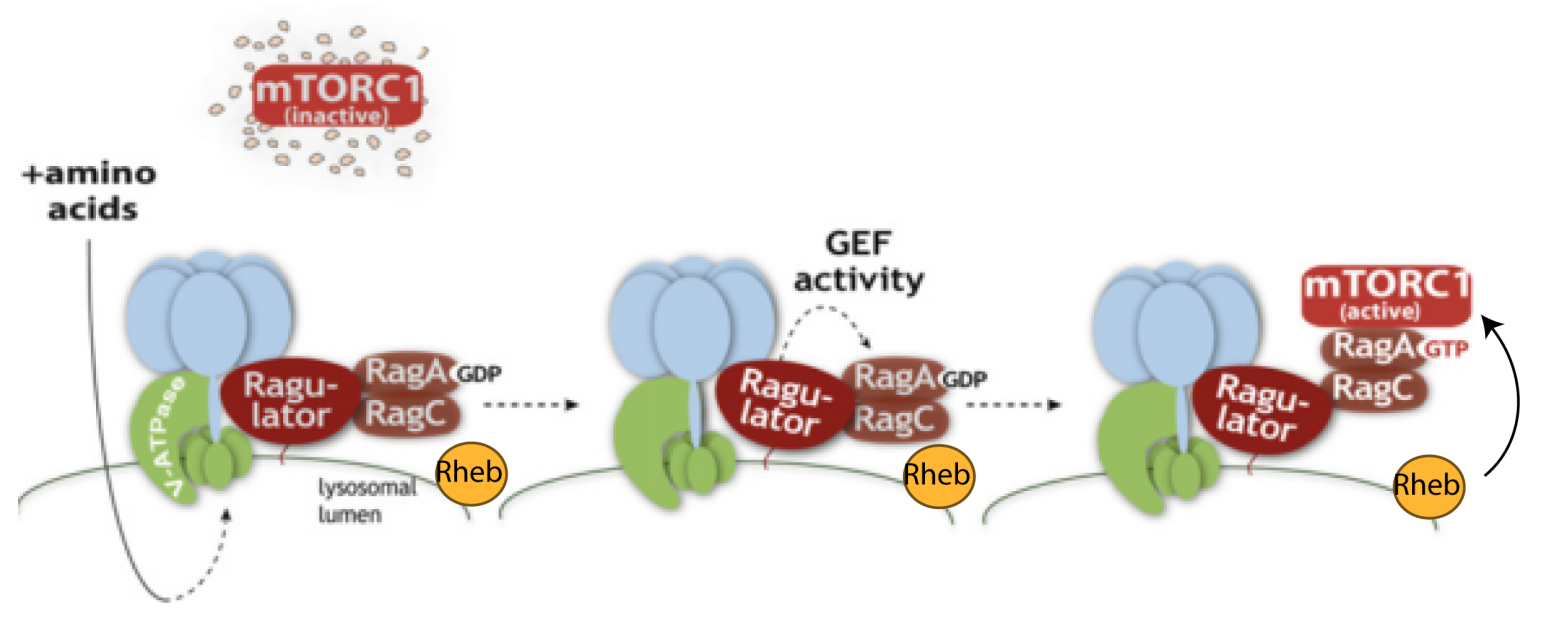


Chapter 2

Experimental Procedures 


\section{1: Cell Culture}

The macrophage-like murine cell lines RAW264.7 and J774 were respectively grown in RPMI 1640 and DMEM media supplemented with 5-10\% fetal bovine serum (Wisent, St. Bruno, QC) at $37{ }^{\circ} \mathrm{C}$ and $5 \% \mathrm{CO}_{2}$. Mouse bone marrow-derived dendritic cells were harvested from wild-type female 7-9 week old C57BL/6J mice (Jackson Laboratories, Maine, USA) or from MHC-II-GFP knock-in mice (Boes et al., 2002) as previously described (Inaba et al., 1992) with minor modifications. Briefly, the bone marrow was flushed from leg bones with phosphate-buffered saline (PBS) using a 25G syringe and red blood cells were lysed with a brief hypoosmotic treatment. Cells were plated at $2 \times 10^{6}$ per well in 4 mL DMEM supplemented with $10 \%$ fetal bovine serum, $55 \mu \mathrm{M}$ 2-mercaptoethanol, $10 \mathrm{ng} / \mathrm{mL}$ recombinant mouse GM-CSF (PeproTech, Rocky Hill, NJ) and penicillin/streptomycin antibiotics (Pen/Strep from Wisent). Cells were washed every 2 days by replacing half the medium with fresh medium. Experiments were conducted on days 7-9. All animals were used following Institutional ethics requirements.

\section{2: Transfection and Plasmids}

Plasmid transfections of cells were carried out by treating cells with $2 \mu \mathrm{g}$ plasmid DNA and $5 \mu \mathrm{L}$ Fugene HD (Roche) as per the manufacturer's protocol. Cells were allowed to recover for $16 \mathrm{~h}$ prior to imaging. The plasmids encoding GFP-Rab7,

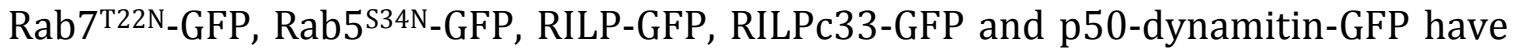
previously been described (Bucci, Thomsen, Nicoziani, McCarthy, \& van Deurs, 2000; Cantalupo, Alifano, Roberti, Bruni, \& Bucci, 2001; Echeverri, Paschal, Vaughan, \& Vallee, 1996; Vieira et al., 2003). Plasmids encoding Arl8b-GFP and Arl8b ${ }^{\mathrm{T} 24 \mathrm{~N}}$-GFP were characterized previously (Kaniuk et al., 2011). mCherry-FYC01 was a kind gift from Terje Johansen (University of Tromsø, Norway) (Pankiv et al., 2010). KIF5 ${ }^{\text {DN }}$ RFP was generously provided by Rene Harrison (University of Toronto Scarborough, Toronto, ON, Canada) (Silver \& Harrison, 2011). 
2.3: Gene Silencing by siRNA Electroporation

Gene silencing in RAW cells by siRNA oligonucleotides was conducted using electroporation. Briefly, RAW cells were grown to confluence in a T25 flask. Cells were then scraped, pelleted and resuspended in $90 \mu$ Amaxa electroporation solution (Lonza, Switzerland) and $5 \mu \mathrm{l}$ of $20 \mu \mathrm{M}$ SMARTpool set containing four siRNA oligonucleotides against a target gene or individual siRNA oligos selected from the SMARTpool mix. SiRNA against the following mouse genes was used: FYC01 (Gene ID: 17281, SMARTpool: ORF L-055432-01, individual oligos: ORF J 055432-09, ORF J-055432-11), RILP (Gene ID: 280408, individual oligos: ORF J 063866-11, ORF J-063866-12), Arl8B (Gene ID: 67166, SMARTpool: 3_-UTR/ORF L 056525-01, individual oligos: ORF J-056525-10, 3_-UTR J-056525-12), SKIP (Gene ID: 69582, SMARTpool: ORF L-050865-00, individual oligos: ORF J-050865-05, ORF J-050865-07), mTOR (Gene ID: 56717, SMARTpool: ORF L-065427-00-0005) or Ulk1 (Gene ID: 22241, SMARTpool: ORF L-040155-00-0005) all from Dharmacon, Lafayette, CO. As a control, a non-targeting SMARTpool siRNA oligonucleotide set was used (D-001810-10, Dharmacon, Lafayette, CO). To optimize target knockdown, cells were electroporated with either a single or double pulse separated by one minute with an Amaxa Nucleofector 1 Electroporator and incubated for either 24 or 48 hours to allow for gene silencing.

\section{4: SDS-PAGE and Western Blotting}

Total cell lysates were prepared by resuspending and homogenizing cells in lysis buffer consisting of protease (cOmplete, mini, EDTA-free) and phosphatase (PhosSTOP) inhibitor cocktail tablets from Roche (Indianapolis, IN) dissolved in PBS $+0.1 \%$ Triton X-100. Cell lysates were cleared by centrifuging for $10 \mathrm{~min}$ at 18,000 g. The supernatant was then mixed with $2 x$ Laemmli sample buffer (Bio-Rad, Hercules, CA) and boiled for 5 minutes. Lysates were then loaded and separated in a 12\% SDS-PAGE and transferred onto a PVDF membrane (Bio-Rad, Hercules, CA). 
The membranes were incubated with primary antibodies, followed by HRP-linked secondary antibodies in Tris Buffered Saline $+0.1 \%$ Tween-20 containing 5\% nonfat milk or bovine serum albumin (BSA) (BioShop Canada, Burlington, ON), followed by enhanced chemiluminescence detection using the Amersham ECL Western Blotting Detection Reagent and high-performance chemiluminescence film from GE Healthcare (Buckinghamshire, UK). Western blots were quantified using the software program ImageStudio (Version 3.1.4, LI-COR, Lincoln, NE).

Primary antibodies used for Western blotting were rabbit antiserum against Arl8B which was a kind gift from Dr. Michael Brenner (Brigham and Women's hospital, Boston, MA, USA) (Garg et al., 2011), rabbit anti-mTOR, rabbit anti-S6K, rabbit anti-pS6K, rabbit anti-Akt, rabbit anti-pAkt, rabbit anti-pIRF3 (Cell Signaling, Danvers, MA), all used at 1:1000 dilution, mouse anti-GAPDH antibodies (Millipore, Billerica, MA), used at 1:10000 dilution and Bett polyclonal antibody against MHC-II $\beta$ chain which was a kind gift from Dr. Shin (UCSF, California). Secondary antibodies were HRP-linked goat anti-rabbit or donkey anti-mouse from Jackson ImmunoResearch (West Grove, PA).

\section{5: Pharmacological Inhibition}

Cells were treated with various compounds throughout this study including $1 \mu \mathrm{M}$ LY294002 (L9908), $2 \mu$ M MRT67307 (SML0702), $5 \mu$ M IRAK1/4 Inhibitor I (I5409), $1 \mu$ M Rapamycin (R8781), 200 nM PP242 (P0037), $10 \mu$ M Nocodazole (M1404), all from Sigma (St. Louis, MO), $200 \mathrm{nM}$ Torin1 (cat \# 4247), $50 \mathrm{nM}$ WYE687 (cat \# 4282), $100 \mu \mathrm{M}$ A769662 (cat \# 3336) from Tocris (Bristol, UK) and $1 \mu \mathrm{M}$ ZSTK474 (S1072) from Selleckchem (Houston, TX). Dyngo 4a was used at $50 \mu \mathrm{M}$ (abcam, ab120689). Typically, inhibitors were added to cells for 20 minutes prior to stimulation with LPS to induce lysosome tubulation. As a control, cells were treated with DMSO. MyD88 inhibitory peptide (Pepinh-MYD) and control peptide (Pepinhctrl) were from InvivoGen (Cat code: tlrl-pimyd) and endocytosed by cells for $3 \mathrm{~h}$ prior to downstream treatments. 
To visualize microtubules, immunostaining was conducted by briefly washing cells in PBS and fixing cells with 4\% paraformaldehyde for $20 \mathrm{~min}$. For mTOR and lysosome staining, cells were briefly washed in PBS and fixed with $0.5 \%$ paraformaldehyde and $0.2 \%$ gluteraldehyde as per a previously established

protocol for preserving tubular lysosomes (Robinson, Chiplonkar, \& Luo, 1996). Cells were washed in $0.5 \%$ BSA/PBS, permeabilized with $0.1 \%$ Triton X-100 in PBS for 5 min and washed again with BSA/PBS. Staining with primary antibodies was conducted at room temperature for $0.5 \mathrm{~h}$. Cells were washed with BSA/PBS, incubated with secondary antibodies for $1 \mathrm{~h}$ and washed a final time before mounting coverslips with Dako fluorescent mounting medium.

Primary antibodies used for immunofluorescence were mouse anti- $\alpha$-tubulin (Sigma), rabbit anti-mTOR (Cell signaling, Danvers, MA), LAMP1 (1D4B antibody from Cell Signaling all used at 1:1000. Secondary antibodies were AlexaFluor488 linked donkey anti-mouse, AlexaFluor488 linked donkey anti-rabbit and Cy3-linked donkey anti-rat, from Jackson ImmunoResearch (West Grove, PA).

\subsection{Flow Cytometry}

BMDCs were washed in 0.5\% BSA/PBS three times and incubated on ice for 30 minutes with primary antibodies (1:100). Cells were washed twice with $0.5 \%$ BSA/PBS and incubated on ice for 20 minutes with secondary antibodies (1:50). Cells were washed with BSA/PBS then fixed in 2\% paraformaldehyde in PBS for 20 minutes. Finally, cells were washed in PBS and stored on ice until analyzed. Flow cytometry was performed at the University of Toronto Flow Cytometry facility on an LSRII flow cytometer (BD Biosciences) and analysis was conducted using FlowJo software (Tree Star, Ashland, OR).

Cells were labeled for flow cytometry with hamster anti-CD11c and rat antiMHC-II primary antibodies from BD Pharmingen (San Jose, CA), followed by Dylight649-linked donkey anti-hamster and AlexaFluor488-linked donkey anti-rat 
from Jackson ImmunoResearch (West Grove, PA).

2.8: Reverse Transcription and Quantitative PCR

To determine relative gene expression of FYC01, RILP, Arl8b, SKIP, Ulk1 and IL-6 we employed mRNA reverse transcription and quantitative PCR (qPCR). Briefly, RNA was extracted using the GeneJET RNA Purification Kit (Thermo Scientific, Cat\#: K0731), as per manufacturer's instructions. Equal amounts of RNA from all conditions were loaded as template for generation of cDNA by reverse transcription PCR using the SuperScript VILO cDNA synthesis kit (Invitrogen, Cat\# 11754050). cDNA was diluted 1:100 and qPCR was conducted using the TaqMan system (Life Technologies) on a Step One Plus Real-Time PCR thermal cycler (Applied Biosystems) with Step One software (Applied Biosystems, v2.2.2). The TaqMan gene expression assays for the reference gene Abt1 (Cat\# 4331182 / Mm00803824 / Life Technologies) and target genes FYC01 (Cat\# 4331182 / Mm00530503 / Life Technologies), RILP (Cat\# 4331182 / Mm01240442 / Life Technologies), Arl8b (Cat \# 4331182 / Mm00482600 / Life Technologies), SKIP (Cat \# 4351372 / Mm01351044 / Life Technologies) Ulk1 (Cat\# 4331182 / Mm00437238 / Life Technologies) or IL-6 (Cat \# 4331182 / Mm00446190 / Life Technologies) were duplexed in triplicate and the TaqMan Fast Advanced Master Mix (4444963) was used. Target gene expression was determined by relative quantification ( $\Delta \Delta \mathrm{Ct}$ method) to Abt1 reference gene and the control sample.

\section{9: Lysosome Tubulation Assays}

In order to visualize lysosomes, cells were allowed to endocytose dextran labeled with AlexaFluor-555 or -488 (Invitrogen) at $100 \mu \mathrm{g} / \mathrm{mL}$ for $0.5-1.5 \mathrm{~h}$, followed by at least a $1 \mathrm{~h}$ chase in dextran-free medium. To induce lysosome tubulation, cells were then treated with $100 \mathrm{ng} / \mathrm{mL}$ LPS (Salmonella minnesota serotype minnesota Re 595; Sigma) for $2 \mathrm{~h}$ prior to imaging or equivalent volume of PBS was used as 
control. LPS was maintained in the medium during imaging. When appropriate, lysosomes were pre-labeled with fluorescent dextrans prior to transfection or electroporation to ensure trafficking of the probe to lysosomes. Where inhibitors were used, cells were pre-treated with compounds at the concentrations indicated for 20 min prior to LPS addition for $2 \mathrm{~h}$, or equivalent volume of DMSO was used as control followed by LPS treatment. Cells were imaged live following treatments and LPS/inhibitors/DMSO/PBS were maintained in the medium during imaging. TLs were quantified manually by counting all lysosomal tubules that were $\geq 4 \mu \mathrm{m}$ in length. Lastly, and because of significant variation in the actual number of tubules from day-to-day, we developed a tubulation index where the number of tubules in any given condition within a specific day is normalized against number of tubules found in cells treated with LPS for $2 \mathrm{~h}$. For the kinetics of lysosome tubulation, cells were treated with $100 \mathrm{ng} / \mathrm{mL}$ LPS and imaged live at various time points.

\subsection{0: Timelapse Microscopy and Particle Tracking}

Live-cell images were acquired using a Quorum Wave-FX spinning disk confocal microscope (Quorum Technologies) based on a Zeiss Axiovert 200M fitted with a Yokogawa CSU10 spinning disk head and a back-thinned electron multiplier camera (C9100-13 ImagEM; Hamamatsu). Timelapse microscopy was carried out by capturing images every 0.5 seconds for $2-3$ min using the $63 \mathrm{x}$ oil immersion objective and a $37^{\circ} \mathrm{C}$ stage. Images were acquired and analyzed with VOLOCITY software (version 4.4; PerkinElmer). Cells were kept in RPMI 1640 (Wisent) media supplemented with 5\% FBS and buffered with HEPEs during live-cell imaging.

TLs and punctate lysosomes were tracked manually in each frame in VOLOCITY using the 'manual tracking with the region of interest' function for a minimum of 120 frames. Mean velocity measures and averages speed during both motile and non-motile phases of lysosomes. Displacement measures the distance between starting and end positions during an observation period. Total track length measures total distance traveled during the observation period. Image processing 
was done with CS3 Adobe Photoshop (version 10.0) and Illustrator (version 13.0.0).

\subsection{1: Lysosomal Positioning Assays}

Lysosomes of RAW 264.7 cells were labeled and cells were either left untreated or were treated with $200 \mathrm{nM}$ Torin 1 for $1 \mathrm{~h}$. Cells were either kept in regular Ringer's medium ( $\mathrm{pH}$ 7.2) for $20 \mathrm{~min}$, Acetate Ringer's ( $\mathrm{pH}$ 6.8) for 20 min or Acetate Ringers for 20 minutes followed by regular Ringers medium for 20 min (Ringer's and Acetate Ringer's solutions were prepared as per Heuser, 1989). Cells were briefly washed in PBS, fixed in 4\% paraformaldehyde and mounted onto glass slides with Dako fluorescent mounting medium. Cells were imaged by confocal microscopy and z-stacks were acquired.

Lysosomal positioning was quantified using ImageJ software (National Institutes of Health, Bathesda, MD). Briefly, z-stacks were collapsed to give an extended view of all lysosomes in cells. Each cell was outlined manually to generate a region of interest (shell) and the same shell was reduced in size by 15 pixel iterations to produce 3 shells in total, per cell. Thresholding for lysosomal signal was conducted manually and the lysosomal area was computed in each shell and normalized to shell area.

\subsection{Image Quantification of LC3, RFP-Rab7 and Arl8b-GFP}

To determine if Ulk1 silencing affected autophagy, control and Ulk1-silenced cells were either untreated or treated with torin 1 for 2 hours. Immunofluorescence against LC3 was conducted (described above) and images of cells were acquired (zstacks) by spinning disk confocal microscopy. Cells were analyzed for average number of LC3 puncta, representing autophagosomes. Briefly, using ImageJ software $(1.46 \mathrm{r}, \mathrm{NIH}), \mathrm{z}$-stacks were collapsed to provide an extended view of each field and individual cells were cropped. A manual threshold was applied to all cells to exclude all cytosolic signal, which did not appear punctate. Using the 'analyze 
particles' tool, the 'count' was obtained, which identified the number of LC3 puncta. This was done for each cell for each condition (at least 15 cells per condition across 2 experiments).

For quantification of membrane-to-cytosol distribution of Arl8b and Rab7, cells were transfected with Arl8b-GFP, RFP-Rab7 and Rilpc33-GFP plasmids using Fugene HD (Promega) as per manufacturer's instructions. After each treatment, live-cell imaging with spinning disc confocal microscopy was performed. Subsequently, each image was analysed using ImageJ by manually applying a threshold outlining only the membrane-bound fluorescence (punctate), creating a mask. The mean fluorescence intensity under this mask was then subjected to Analyze Particle function, followed by background subtraction. Next, the mean intensity of the cytosol was obtained by drawing regions of interest in the cytosolic pool, followed by background subtraction. The average ratio of membrane-bound to cytosolic fluorescence intensity was then calculated for each treatment (This method was performed by Victoria Hipolito).

\subsection{3: Statistical Analyses}

All experiments were repeated independently at least three times. In some cases, due to variation in the absolute number of TLs in any given day (but not within the same day), data shows normalized mean values against the corresponding control. Measurements of lysosomal tubulation across various conditions (multiple groups) were subject to analysis of variance (ANOVA) with Tukey's post-hoc test. Statistical analysis of differences between two groups under an experimental condition was done using a Student's t-test. A p value of $<0.05$ was considered significant. Data are presented as mean \pm standard error of the mean (SEM), unless stated otherwise. For qRT-PCR analysis of gene silencing, we employed one-sided Student's t-test. 
Chapter 3

Results 
3.1: Rab7 and Arl8 GTPases are Necessary for Lysosome Tubulation in Macrophages

3.1.1: LPS-Stimulated Macrophage Cell Lines Form Highly Dynamic Lysosome Tubules

The study of tubular lysosomes in activated macrophages has been challenging in part due to the fact that primary macrophages are not easily amenable to common methods of genetic manipulation such as transfection. Thus, to enable the characterization of tubular lysosomes, we developed a model system for this purpose. Using the mouse macrophage cell lines RAW 264.7 and J774, which have been widely employed in the study of phagocytosis and phagosome maturation (Beemiller et al., 2010; Garg et al., 2011; Harrison, Bucci, Vieira, Schroer, \& Grinstein, 2003; Hoffmann et al., 2010), we tested whether LPS activation induced tubulation of lysosomes, as was previously observed in dendritic cells with tMIIC. We proceeded to do this by fluorescently labeling lysosomes with AlexaFluor555conjugated dextran, which was taken up by cells for 30 min through endocytosis and following a 1-hour chase, accumulated in lysosomes. Resting macrophages exhibited predominantly punctate lysosomes $(<4 \mu \mathrm{m}$ in length) under the fluorescence microscope (Figure 3.1A, Figures section at the end of this chapter). On average, these cells exhibited one tubular lysosome per cell ( $\geq 4 \mu \mathrm{m}$ in length) (Figure 3.1C). In contrast, macrophages stimulated with 100ng/mL LPS for 2 hours displayed extensive tubulation of lysosomes (Figure 3.1A), where the number of cells with at least one TL increased from $50 \%$ to about $85 \%$ (Figure 3.1B) and there was approximately a threefold increase in the average number of TLs per cell (Figure 3.1C). Due to variation in the extent of tubulation between experiments, but not within, the tubulation values were normalized to control conditions. A complete listing of absolute values corresponding to results in section 3.1 is presented in Table A-1 of the Appendix. Overall, both RAW and J774 cell lines underwent lysosome tubulation when exposed to LPS. Despite a limited resolution with spinning disk confocal microscopy, tubular lysosomes were observed to be very dynamic, frequently elongating, collapsing, fusing and branching (Figure 3.1D). 


\subsection{2: Dynein and Kinesin I are Required for Lysosome Tubulation}

The observation that TLs are highly dynamic suggests the involvement of microtubules and the microtubule-associated motor proteins dynein and kinesin in their formation. Indeed, the microtubule depolymerizing agent nocodazole caused lysosomal tubules to fragment and collapse (Figure 3.2A). This observation is in line with previous literature reports that microtubules were necessary for TLs and tMIICs in macrophages and DCs (Swanson, Burke, \& Silverstein, 1987; Vyas et al., 2007). To test the requirement of each motor protein in TL biogenesis, we examined TL formation under conditions where proper function of each was hindered. Kinesin I, encoded by KIF5, has been shown to associate with lysosomes (Tanaka et al., 1998), therefore we overexpressed a previously characterized kinesin I dominant negative mutant fused to red fluorescent protein (KIF5 ${ }^{\mathrm{DN}}$-RFP) (Silver \& Harrison, 2011). Cells overexpressing this construct were strongly hindered in their ability to form tubular lysosomes with $41 \pm 5 \%$ fewer tubular lysosomes than control, untransfected cells (Figure 3.2B, D).

To disrupt dynein, we overexpressed p50 dynamitin, a subunit of dynactin, which is required for proper dynein function but causes disassembly of the dyneindynactin complex when expressed exogenously (Burkhardt, Echeverri, Nilsson, \& Vallee, 1997). LPS-treated cells overexpressing dynamitin-GFP exhibited a reduction of $38 \pm 6 \%$ in the number of TLs per cell relative to untransfected cells (Figure 3.2C, D). Co-expression of KIF5 ${ }^{\mathrm{DN}}$-RFP and dynamitin-GFP did not have an additive effect. This may be due to plasmid co-transfections not necessarily reflecting same level of expression of the individual mutant proteins as single transfections. Taken together, these data indicate that tubular lysosome biogenesis is dependent on both dynein and kinesin I microtubule motor proteins, results that are consistent with the dynamic nature of this organelle. 


\subsection{3: Rab7 is Necessary for Lysosome Tubulation}

Dynein assembly on lysosomes is controlled by the lysosomal small GTPase Rab7, which nucleates a complex between RILP, ORP1L and dynactin (Johansson et al., 2007; Jordens et al., 2001). Furthermore, Rab7 has been shown to control kinesin activation on lysosomes via the effector protein FYCO1, though direct interaction between these two proteins has not yet been detected (Pankiv et al., 2010). Given these roles in lysosomal trafficking, we examined whether this GTPase was necessary for the formation of tubular lysosomes. We first made use of a GFP-Rab7 construct to determine whether it was expressed on tubular lysosomes. Indeed, GFP-Rab7 co-localized with Alexa-555-labeled TLs after LPS treatment of macrophages (Figure 3.3A). What is more, the GTP-bound form of Rab7 can be visualized by expressing RILP-c33-GFP - this fragment of RILP binds to GTP-Rab7 but lacks domains required for interaction with dynein, preventing aggregation of lysosomes (Cantalupo, Alifano, Roberti, Bruni, \& Bucci, 2001). RILP-c33-GFP was visible on tubular lysosomes indicating that GTP-bound Rab7 exists on TLs (Figure 3.3B). Finally, to determine whether or not Rab7 was required for TL formation, we overexpressed a dominant negative mutant GFP-Rab7T22N. As shown in Figure 3.3C and 3.3D, cells expressing GFP-Rab7 ${ }^{\mathrm{T} 22 \mathrm{~N}}$ had $67 \pm 5 \%$ fewer TLs per cell than control cells expressing GFP-Rab7. Collectively, these results indicate that Rab7 is important in TL biogenesis.

\subsection{4: RILP is Involved in Lysosome Tubulation}

The findings that Rab7, dynein and kinesin were all necessary for lysosome tubulation to occur, suggested that links between Rab7 and the motors were required. As indicated above, Rab7 can modulate dynein and kinesin activity on lysosomes through the effector proteins RILP and FYC01 respectively. Therefore, we explored the role of these effectors in lysosome tubulation. By overexpressing RILP, lysosomes can be clustered in the perinuclear region near the MTOC, due to hyperstimulation of dynein (Cantalupo, Alifano, Roberti, Bruni, \& Bucci, 2001; 
Jordens et al., 2001). Indeed, overexpressing a GFP-tagged version of RILP induced lysosomal clustering in macrophages (Figure 3.4A). Importantly, this overexpression impaired lysosome tubulation where GFP-RILP transfected cells exhibited $51 \pm 7 \%$ fewer TLs than untransfected control cells (Figure 3.4B). Strikingly, tubules that escaped this inhibitory effect and managed to form in transfected cells were decorated with GFP-RILP and this is in line with the observation that active Rab7 is also present on lysosomal tubules (Figure 3.4A).

We further complemented this overexpression technique with siRNA gene silencing against RILP to determine if loss of RILP affected TL biogenesis. To quantify RILP silencing, we employed quantitative reverse transcription-polymerase chain reaction (qRT-PCR) to determine the relative amount of RILP mRNA, as viable anti-RILP antibodies were not available at the time to detect expression knockdown by western blotting for RILP protein. Using this technique, we show that two individual sets of siRNA oligonucleotides (oligos) against RILP, siRILP oligo 11 and 12 , caused a reduction of $54 \pm 11 \%$ and $52 \pm 8 \%$ in RILP expression, respectively, compared to control cells electroporated with scrambled siRNA oligos (Figure 3.4 C). Most importantly, cells silenced for RILP expression with either of these oligos demonstrated an approximately 60\% reduction in the number of TLs per cell, relative to the control scrambled condition (Figure 3.4D). Taken together, these results suggest that RILP, a Rab7 effector, is involved in TL biogenesis, likely by regulating the dynein motor activity on lysosomes.

\subsection{5: FYC01 Plays a Role in Lysosome Tubulation}

As previously discussed, FYCO1 is another Rab7 effector, thought to modulate the motor activity of kinesin on lysosomes. Thus, we proceeded to define its role in lysosome tubulation. As with RILP, we first applied overexpression of an mCherrytagged FYCO1 construct in macrophages. This was previously shown to force lysosomes and autophagosomes to the cell periphery, presumably through kinesin (Pankiv et al., 2010). Indeed, overexpression of mCherry-FYCO1 caused a 
centripetal distribution of lysosomes labeled with Alexa-488-dextran in macrophages (Figure 3.5A,B). Importantly, this overexpression was accompanied by a robust decrease of $62 \pm 5 \%$ in lysosome tubulation compared to untransfected control macrophages (Figure 3.5C). As with RILP overexpression, FYCO1 was also localized to TLs that were able to form, albeit weakly (Figure 3.5B), and as with RILP, the inhibition of TL formation may be due to pleiotropic effects such as overstimulation of kinesin, as evidenced by peripheral clustering of lysosomes.

We further complemented overexpression of FYCO1 with silencing of the FYC01 gene using siRNA, to determine its requirement in TL biogenesis. Once again, we employed qRT-PCR to determine FYCO1 silencing efficiency due to lack of antiFYCO1 antibodies. First, we show that a SMARTpool oligo mix, or two individual FYCO1-specific siRNA oligos (siRNA oligos 9 and 11) could knockdown FYCO1 expression by $54 \pm 4,46 \pm 7 \%$ and $32 \pm 10 \%$ respectively, compared to the corresponding control cells treated with scrambled siRNA (Figure 3.5D). Most significantly, cells silenced for FYCO1 expression with either the SMARTpool mix, or oligo 9 or 11 , respectively, produced $30 \pm 5 \%, 47 \pm 6 \%$ and $47 \pm 9 \%$ fewer TLs than corresponding controls (Figure 3.5E). These results indicate that FYCO1 is necessary for lysosome tubulation and suggests a model by which Rab7 recruits both RILP and FYCO1 to modulate dynein and kinesin respectively to facilitate lysosome tubulation (refer to model in Figure 3.17).

\subsection{6: Arl8b and SKIP are Necessary for Lysosome Tubulation}

In addition to the Rab7-FYCO1 link, lysosomal kinesin activity can be regulated by the lysosomal small GTPase Arl8b, through interaction with its effector SKIP, a kinesin-interacting protein (Bagshaw, Callahan, \& Mahuran, 2006; Behnia \& Munro, 2005; Hofmann \& Munro, 2006; Rosa-Ferreira \& Munro, 2011). We reasoned that this regulatory arm may also play a role in tubulating lysosomes, thus we investigated whether Arl8b and SKIP are necessary for this phenomenon as well. 
Like GFP-Rab7, we first show that Arl8b-GFP localized to lysosomal tubules (Figure

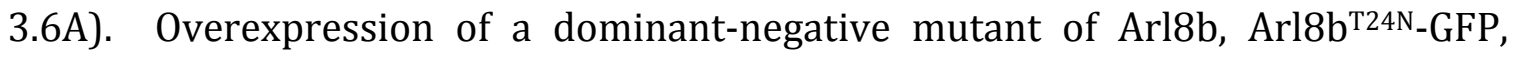
strongly inhibited TL biogenesis in LPS-stimulated macrophages, compared to Arl8b-GFP (Figure 3.6B, C).

To complement overexpression of this dominant-negative mutant, we applied siRNA-mediated gene silencing of Arl8b to determine if loss of endogenous protein affected TL formation. First, we detected a strong knockdown of more than 90\% of Arl8b at the protein level using anti-Arl8b antibodies and western blotting, compared to control cells treated with scrambled siRNA oligos (Figure 3.6D). Second, cells electroporated with SMARTpool siRNA oligo mix against Arl8b showed a drop of $69 \pm 4 \%$ in TLs relative to control (Figure 3.6F). To ensure specificity of the SMARTpool oligo mix, we further assessed the effect of two independent siRNA oligos against Arl8b. Using qRT-PCR, we showed that siRNA oligos 10 and 12 abated Arl8b expression to $23 \pm 4 \%$ and $24 \pm 3 \%$ of control cells (Figure $3.6 \mathrm{E}$ ) and abated TL formation by $58 \pm 5 \%$ and $51 \pm 7 \%$, respectively (Figure $3.6 F$ ). These observations suggest that in addition to Rab7, the lysosomal small GTPase Arl8b is essential for TL formation.

On this line of reasoning, we tested if SKIP, which couples Arl8b and kinesin on lysosomes, was necessary for TL biogenesis. As before, we could show that cells electroporated with an siRNA SMARTpool or two individual oligos (5 and 7), depleted endogenous SKIP expression by $68 \pm 2 \%, 57 \pm 5 \%$ and $36 \pm 7 \%$ respectively, relative to control cells (Figure 3.6G). Consequently, SKIP-silenced cells, in the same order as indicated, suffered a loss of $74 \pm 5 \%, 51 \pm 6 \%$ and $49 \pm 8 \%$ of TLs respectively, relative to cells electroporated with corresponding scrambled siRNA oligos (Figure 3.6H). Together, these results strongly suggest that SKIP, along with Arl8b, is necessary for TL biogenesis. 
3.1.7: Lysosome Tubules are More Motile than Punctate Lysosomes

Lysosomes are dynamic organelles and can frequently undergo bidirectional movement along microtubules, but also exhibit periods of no movement (Cordonnier, Dauzonne, Louvard, \& Coudrier, 2001; Falcón-Pérez, Nazarian, Sabatti, \& Dell'Angelica, 2005; Luzio, Pryor, \& Bright, 2007). Little is known about how tubulation of lysosomes affects their motility. Visually, it appears as though tubular lysosomes in macrophage cell lines are highly dynamic (Figure 3.1D) and more so than punctate lysosomes. To test this, we employed particle tracking of individual random lysosomes and mapped their motilities to quantify and compare various parameters of punctate lysosomes and tubular lysosomes. As expected, punctate lysosomes and TLs often exhibited bidirectional movement (Figure 3.7A, B; tracks 15). Strikingly, TLs underwent significantly greater directional displacement compared to punctate lysosomes, as can be appreciated from tracks in Figure 3.7B compared to Figure 3.7A. Punctate lysosomes were more often static and underwent small vibrational movements (compare Figure 3.7A and Figure 3.7B). Using tracking software, we show that over a one-minute period, TLs were displaced by $3.96 \pm 0.47 \mu \mathrm{m}$ from their starting position compared to only $1.29 \pm 0.31 \mu \mathrm{m}$ for punctate lysosomes (Figure 3.7C). We further examined total track length, as displacement does not take into account overall distance traveled. Once again, TLs exhibited longer track lengths of $11.8 \pm 1.0 \mu \mathrm{m}$ relative to punctate lysosomes with 7.8 $\pm 0.6 \mu \mathrm{m}$ tracks (Figure 3.7D). A significant portion of punctate lysosome track lengths was likely due to small distances traveled during stochastic or vibrational movement of lysosomes. Finally, we measured mean velocity of punctate and tubular lysosomes. We found that the mean velocity of tubules was significantly larger than that of punctate lysosomes (Figure 3.7E). In LPS-treated cells punctate lysosomes moved at $0.16 \pm 0.024 \mu \mathrm{m} / \mathrm{sec}$, whereas TLs in the same LPS-treated cells moved at $0.36 \pm 0.039 \mu \mathrm{m} / \mathrm{sec}$ (Figure 3.7E; dark grey bars). A similar result was obtained between punctate and tubular lysosomes in non-LPS-treated cells (Figure 3.7E; light grey bars) suggesting that the difference in mean velocity between TLs and punctate lysosomes is not attributable to LPS treatment. However, mean 
velocity represents speed of both moving and static phases of lysosomes. When we quantified speed of moving-only lysosomes, both puncta and tubules exhibited a similar speed of $0.61 \pm 0.039 \mu \mathrm{m} / \mathrm{sec}$ and $0.60 \pm 0.18 \mu \mathrm{m} / \mathrm{sec}$, respectively. These results suggest that TLs are more often 'on the move' than punctate lysosomes. Taken together, these results indicate that TLs are generally a more motile population of lysosomes and that LPS-activated macrophages have a larger number of these tubular, motile lysosomes. 
3.2: mTOR Regulates Lysosome Tubulation and Antigen Presentation in Macrophages and Dendritic Cells

3.2.1: TLR4-Induced Lysosome Tubulation in Macrophages Requires MyD88 and PI3-Kinase

As shown in section 3.1, LPS exposure converts punctate lysosomes into a tubular network of lysosomes in macrophages. Other than a requirement for the TLR4 adapter protein MyD88 in LPS-induced MIIC tubulation in dendritic cells (Boes et al., 2003), little is known about the downstream signaling pathway responsible for this phenotype. We sought to fill this gap of knowledge by applying pharmacological antagonists targeting MyD88-dependent and MyD88-independent pathways downstream of TLR4, and assessed their impact on lysosome tubulation in RAW macrophages. The various inhibitors used and their targets are schematically represented in Figure 3.8. As before, lysosomes were pre-labeled by a pulse of fluorescent dextran, followed by a 1 hour chase and lysosome tubulation was stimulated by exposing cells to $100 \mathrm{ng} / \mathrm{mL}$ LPS for 2 hours. Lysosome tubulation was assessed by confocal fluorescence microscopy. Lysosome tubulation data was normalized to the LPS condition.

LPS exposure induced a 2-fold increase in the average number of tubular lysosomes per cell compared to unstimulated cells (Figure 3.9A, B). Pre-treatment of cells with the MyD88-antagonistic peptide PepinhMYD significantly abated lysosome tubulation in response to LPS, while a control peptide had no effect (Figure 3.9A, B). In contrast, blocking the kinase TBK1 with MRT67307 had little effect on LPS-induced lysosome tubulation (Figure 3.9A, B). To ensure that this drug did indeed block TBK1 activity, we detected decreased phosphorylated IRF3, a substrate of TBK1, in response to LPS (Appendix Figure A-1, A). This data suggests that LPS-induced lysosome tubulation proceeds through the MyD88 signaling arm downstream of TLR4. 
Because MyD88 can stimulate IRAK1/4 and PI3K following LPS stimulation (Burns et al., 2003; Laird et al., 2009; Suzuki et al., 2002), we applied inhibitors of each of these kinases to elucidate their roles in TL formation. Inhibition of IRAK1/4 did not affect TL formation in RAW macrophages (Figure 3.9A, B). To test that the IRAK1/4 inhibitor indeed blocked its target, we measured transcription levels of IL6, a cytokine expressed upon TLR stimulation of the IRAK1/4-NFKB pathway (Suzuki et al., 2002; Thomas et al., 1999). Indeed, IL-6 transcription was strongly attenuated with IRAK1/4 inhibitor (Appendix Figure A-1, B). These data suggest that IRAK1/4 is dispensible for lysosome tubulation. In contrast, lysosome tubulation was strongly impaired in LPS-stimulated cells which were treated with LY294002, a general PI3K inhibitor, or the Class I PI3K inhibitor ZSTK474 (Figure 3.9A, B). Overall, the data suggests that LPS signals via a TLR4-MyD88-Class I PI3K pathway to induce lysosome tubulation.

3.2.2: The Canonical PI3K-Akt Pathway Regulates LPS-Induced Lysosome Tubulation

The generation of $\mathrm{PI}(3,4,5) \mathrm{P}_{3}$ at the inner leaflet of the plasma membrane via active PI3K, recruits the signaling protein Akt via its PH domain (Bellacosa et al., 1998). Having observed a strong impediment on lysosome tubulation in cells inhibited for PI3K, we asked whether Akt played a role in lysosome tubulation. First, we characterized the signaling pathway to determine whether LPS indeed triggered the PI3K-Akt pathway in RAW cells. LPS increased the levels of phospho-Akt in cells, a read-out for activation (Figure 3.10A). In addition, Akt activation was independent of IRAK1/4 and TBK1, but required PI3K (Figure 3.10A) and MyD88 (Appendix Figure A-1, C).

We then assessed the requirement of Akt in LPS-induced lysosome tubulation. Pre-treatment of cells with an Akt inhibitor, Akti, followed by LPS, blocked lysosome tubulation by $70 \%$ relative to LPS condition alone (Figure 3.10B, C). These results illustrate that Akt, which is activated by LPS in a PI3K-dependednt manner, is necessary for lysosome tubulation. 
3.2.3: mTOR is Required for LPS-Induced Lysosome Tubulation in Macrophages

mTOR is a key downstream effector in the PI3K-Akt pathway. Thus, we asked whether it is involved in LPS-mediated lysosome tubulation. First, we assessed mTOR activation in LPS-stimulated cells. Since lysosome tubulation occurs at 1-2 hours post-LPS exposure in RAW cells, we sought to determine whether mTOR is activated within this timeframe. We performed western blotting to probe the level of phosphorylated S6K, a canonical target of mTOR (Isotani et al., 1999; Saitoh et al., 2002). Indeed, phospho-S6K levels were enhanced by $77 \%$ and $78 \%$ after 1 and $2 \mathrm{~h}$ of LPS exposure, respectively, relative to control cells (Figure 3.11A). As expected, this effect was completely abolished by torin1 (Figure 3.11A). In addition, and consistent with a role for the PI3K-Akt-mTOR axis but not for IRAK1/4 or TBK1, PI3K inhibition, but not IRAK1/4 or TBK1, abolished LPS-mediated increase in phospho-S6K levels (Figure 3.11B). We could localize mTOR to lysosomal tubules by immunofluorescence microscopy (Appendix Figure A-2, A) and most importantly, when we assessed lysosome tubulation, cells treated with torin1 prior to LPS addition were severely impaired in their ability to form tubular lysosomes (Figure 3.11C, D). Similar results were obtained when various other mTOR antagonists were applied, including rapamycin, PP242 or WYE687 (Appendix Figure A-2, B). These results suggest that mTOR, which is activated by LPS in a PI3K-dependent manner, is required for LPS-induced lysosome tubulation as well.

To complement the pharmacology-based findings, we employed siRNA gene silencing against mTOR to determine if depletion of endogenous protein also affects lysosome tubulation. We electroporated RAW cells with a pool of four siRNA oligos against mouse mTOR, which were then stimulated with LPS and scored for lysosome tubulation. Western blot analysis confirmed that mTOR silenced cells expressed less than $40 \%$ mTOR protein relative to control cells treated with a non-targeting pool of siRNA oligos (siNTP) (Appendix Figure A-2, C). As with pharmacological inhibition, silencing mTOR resulted in impaired lysosome tubulation in RAW cells (Figure 3.11E, F). 
We further sought to inhibit mTOR function in an independent way by activating AMP-kinase (AMPK). This cellular energy sensor is activated when the ATP:ADP ratio is low, leading to arrest of anabolic processes and a shift to catabolic processes like autophagy (reviewed in Hardie, Ross, \& Hawley, 2012). Notably, activation of AMPK increases the activity of the mTOR repressor TSC1/2 and results in phosphorylation of Raptor, a component of mTORC1, which impairs mTOR complex assembly and function (Gwinn et al., 2008; Inoki, Zhu, \& Guan, 2003). Thus, AMPK activation is inversely related to mTOR activation. Therefore, we activated AMPK using the agonist A769662, which in turn inhibits mTOR (Appendix Figure A2, D), and scored lysosome tubulation. This method of mTOR inhibition also strongly blocked TL biogenesis in response to LPS (Figure 3.11G, H). Overall, these results demonstrate that mTOR is required for LPS-induced lysosome tubulation in RAW cells.

\subsection{4: The Akt-mTOR Axis Prolongs LPS-Derived Signaling in Macrophages}

Following exposure to LPS, cells quickly internalize the TLR4-MyD88 complexes, which are transported to endosomes for inactivation (Husebye et al., 2006). This led us to question whether the Akt-mTOR pathway may serve to sustain LPSinduced signaling after TLR4 degradation. To test this, we examined the kinetics of phospho-Akt and phospho-S6K to assess Akt and mTOR activation after LPS exposure, respectively. Interestingly, both were significantly stimulated within 15 minutes of LPS exposure and remained strongly upregulated for at least 2 hours (Figure 3.12A).

To determine if this activation correlates with lysosome tubulation, we tested the kinetics of lysosome tubulation. Interestingly, significant lysosome tubulation occurred within only 30 minutes of LPS exposure and gradually increased over time (Figure 3.12B, C). These results suggest that the Akt-mTOR axis may help sustain signaling after TLR4 internalization and degradation, and may help program various aspects of macrophages including lysosome morphology and function. Is this a 
conserved phenomenon in other innate immune cells? It is known that primary dendritic cells convert their MIIC, a lysosome-related organelle, into long tubular structures following LPS stimulation, and this is proposed to aid antigen presentation in maturing DCs (Boes et al., 2002; Chow, Toomre, Garrett, \& Mellman, 2002). Thus, we next examined if the role of mTOR on lysosome tubulation extended to dendritic cells as well.

\subsection{5: mTOR Controls Lysosome/MIIC Tubulation in Primary Dendritic Cells}

To test whether mTOR controls lysosome tubulation in other cell types, we extended our experimental analysis to primary mouse bone marrow-derived dendritic cells (BMDCs). To confirm that the tubular MIIC and TLs are the same compartment in BMDCs, we labeled lysosomes of BMDCs from MHCII-GFP-expressing transgenic mice (Boes et al., 2002) with fluorescent dextrans. After LPS stimulation, cells were observed by live-cell imaging. We observed abundant MHCII-GFP-positive tubules that overwhelmingly co-labeled with Alexa-555-dextran, suggesting that the MIIC tubules are indeed lysosomal (Figure 3.13A).

We next demonstrated that LPS treatment increased the levels of phosphoS6K in BMDCs by about $90 \%$ at 1 and $2 \mathrm{~h}$ and this effect was abolished by torin 1 (Figure 3.13B). This result indicates that, as in RAW cells, LPS stimulates mTOR in DCs. LPS activation induced a striking amount of lysosome tubulation in BMDCs with a 20-fold increase over unstimulated BMDCs (Figure 3.13C, D). Finally, we applied mTOR inhibitors and scored lysosome tubulation in BMDCs. Treatment with rapamycin or torin1 prior to LPS stimulation impeded TL formation, as we observed a 5-fold reduction in lysosome tubulation compared to LPS condition alone (Figure 3.13C, D). Overall, these results indicate that mTOR plays an important role in lysosome tubulation in innate immune cells. 
3.2.6: Autophagy Does Not Impact LPS-Mediated Lysosome Tubulation

mTOR inhibition is a key trigger for autophagy (Beugnet, Tee, Taylor, \& Proud, 2003). Therefore, we questioned whether autophagy might antagonize lysosome tubulation in torin1-treated cells. To test this, we employed siRNA-mediated gene silencing against ULK1 (unc51-like autophagy activating kinase 1), the major kinase responsible for autophagy induction. Using this approach, we successfully silenced ULK1 expression by $>60 \%$, as measured by quantitative PCR (Figure 3.14A). To ensure that this level of ULK1 silencing was sufficient to impair autophagy, we examined the number of LC3 puncta that formed in cells by immunofluorescence microscopy, a well-characterized assay for autophagosome formation (Kimura, Fujita, Noda, \& Yoshimori, 2009; Mizushima, Yoshimori, \& Levine, 2010). Using cells electroporated with control non-targeting siRNA, we observed a 3-fold increase in the number of LC3-positive stuctures upon torin1 treatment (Figure 3.14B, C). Remarkably, ULK1-silenced cells strongly resisted the formation of LC3-positive puncta after torin1 treatment (Figure 3.14B, C), indicating that autophagy was indeed suppressed in these cells.

Having shown that silencing ULK1 successfully blocked autophagy, we assessed the effect of torin1 in tubular lysosome biogenesis in these cells. First, ULK1-silenced cells were as proficient at tubulating their lysosomes in response to LPS as control cells electroporated with non-targeting oligonucleotides (Figure 3.14D, E). Most importantly, LPS-induced lysosome tubulation was suppressed by torin1 equally between ULK1-silenced cells and control cells (Figure 3.14D, E). This data suggests that autophagy is not responsible for blocking lysosome tubulation in cells treated with torin1, but rather that mTOR activity is necessary for lysosome tubulation. 


\subsection{7: mTOR is Required for Anterograde Lysosomal Transport}

To address how mTOR influences lysosome morphology, we tested whether there were any apparent changes to the aforementioned lysosome tubulation machinery (Chapter 3, Section 3.1) upon inhibition of mTOR. We began with microtubules, the scaffold along which lysosomes tubulate (Swanson, Bushnell, \& Silverstein, 1987; Vyas et al., 2007). We employed immunofluorescence against $\alpha$-tubulin and compared the general appearance of microtubules in cells with or without functional mTOR. We observed no obvious differences in the overall microtubule organization in RAW cells between control, LPS, Torin1 or Torin1+LPS conditions (Appendix Figure A-3, A). In addition, we observed no difference in basal lysosomal motility upon mTOR inhibition (Appendix Figure A-3, B).

Because microtubules and basal lysosomal motility appeared unaffected, we sought to determine whether motility/position of lysosomes was affected in stimulated cells. In order to examine both kinesin- and dynein- dependent lysosomal transport, we applied a previously established protocol developed by Heuser (Heuser, 1989). By lowering the $\mathrm{pH}$ of the medium with acetate, lysosomes could be forced to the cell periphery, a process that required kinesin activity. This treatment could be followed by a recovery period in which regular medium is replaced. During this time, lysosomes returned to their perinuclear and dispersed distribution, which required dynein. Thus, we assessed the distribution of lysosomes in cells under resting conditions, acidic conditions and following recovery, in both control and mTOR-inhibited cells. To quantify lysosomal distribution, we applied a shell analysis in which the percentage of total lysosomes was computed in three different regions of the cell: near the nucleus, in the cell periphery or the intermediate space between these two areas (schematic in Figure 3.15B). As expected, acetate redistributed lysosomes toward the cell periphery in control cells, as there was a depletion of lysosomes from the perinuclear shell and subsequent accumulation in the peripheral shell (Figure 3.15A, B). We computed redistribution as a ratio of the percentage of lysosomes in the peripheral shell to the percentage of lysosomes in the perinuclear shell, with a high ratio indicating strong 
peripheral redistribution and a low ratio indicating poor peripheral redistribution. Interestingly, although mTOR-inhibited cells behaved similar to control, the extent of peripheral lysosomal distribution following acetate treatment was significantly attenuated (Figure 3.15A, B-compare black and grey bars in acetate conditions). Of note, mTOR-inhibited cells showed a recovery of lysosomes back to the perinuclear shell comparable to that of control cells (Figure 3.15A, B-compare black and grey bars in recovery conditions). Since the acetate condition shows a ratio of only 2.2 in torin1-treated cells compared to 3.8 in control cells, and recovery was comparable between the two, we conclude that anterograde rather than retrograde lysosomal trafficking is likely affected by mTOR. Thus, the results suggest that mTOR may be exerting its effects on lysosome tubulation by directly or indirectly up-regulating kinesin and/or down-regulating dynein activity.

\subsection{8: mTOR is Required for Antigen Presentation in Dendritic Cells}

Others have previously observed tubules containing MHCII-GFP in DCs being targeted to the immunological synapse between host DC and bound T-cell (Boes et al., 2002, 2003). From this, it has been proposed that MHCII is delivered to the plasma membrane for antigen presentation via these tubular MIIC intermediates Barois, de Saint-Vis, Lebecque, Geuze, \& Kleijmeer, 2002; Boes et al., 2002; Chow, Toomre, Garrett, \& Mellman, 2002). We thus asked whether inhibition of mTOR, which impairs TL formation, would also impair MHCII delivery to the plasma membrane of activated DCs. To test this, we treated BMDCs from wild-type C57BL/6J mice with either LPS or LPS and Torin1, and stained for surface MHCII. Using flow cytometry we detected a strong surface accumulation of MHCII in the LPS condition, as expected (Figure 3.16A). In comparison, mTOR-inhibited cells displayed a significantly reduced amount of plasma membrane MHCII after LPS stimulation (Figure 3.16A). This change in surface MHCII was not due to an overall change in MHCII expression as total MHCII levels remained unchanged between treatments (Figure 3.16B, C). Taken together, there results indicate that mTOR, a 
regulator of the morphology and trafficking of lysosomes/MIIC, controls cell-surface delivery of MHCII in BMDCs. In addition, these results remain consistent with a proposed role for tubular MIIC intermediates in delivering antigen to the cell surface for antigen presentation.

\subsection{9: Model of Tubular Lysosome Biogenesis}

Tubular lysosome biogenesis required the concerted actions of lysosomal small GTPases Rab7 and Arl8b, their effectors RILP, FYCO1 and SKIP, and the microtubule motor proteins dynein and kinesin I (Chapter 3, Section 3.1). LPS-induced lysosome tubulation appears to be activated through a TLR4-MyD88-PI3K-Akt-mTOR axis. Although the mechanism of how mTOR affects lysosome tubulation has not been worked out, I provide evidence that mTOR likely impedes on anterograde lysosomal transport, and is therefore necessary for processes like antigen presentation, which rely on lysosome tubulation/anterograde lysosomal transport (Chapter 3, Section 3.2). Thus, I integrated my experimental results from sections 3.1 and 3.2 into a model for tubular lysosome biogenesis (Figure 3.17). In this model, I envision that LPS signals through its receptor TLR4 to activate the MyD88-PI3K-Akt-mTOR pathway. In turn, mTOR integrates these signals to influence lysosomal anterograde transport through modulating activity of microtubule-based motors. As yet, it is unclear whether mTOR acts directly or indirectly on the motors to affect their activity. Finally, motors engage lysosomal movement by linking to lysosomal machinery: kinesin can bind the Arl8b effector SKIP or the Rab7 effector FYCO1 and dynein can bind the Rab7 effector RILP. It is the concerted action of these motors and their links to the lysosome that allow for "stretching" of this organelle along microtubules to bestow a tubular structure upon the lysosome. Finally, the activity of all the components of TLs is required for efficient cell-surface delivery of MHCII in professional antigen presenting cells, as these tubular lysosomes appear to be the compartment that is important for antigen delivery to the plasma membrane. 
Figures for Chapter 3 
Figure 3.1: LPS Stimulates Lysosome Tubulation in Macrophage Cell Lines. (A) Lysosomes of resting (left) and LPS-stimulated (right) RAW macrophages. Arrowheads indicate tubular lysosomes. Insets, bright field; scale bars, $15 \mu \mathrm{m}$. (B) Percentage of cells displaying at least one tubular lysosome, where tubular lysosome $\geq 4$ um. (C) Average number of tubular lysosomes per cell. In both (B) and (C) data are mean \pm SEM of $n=3$ experiments, where $>50$ cells were examined per condition per experiment; means were statistically analyzed by Student's t-test; $\mathrm{p}<0.0001$. (D) Snapshots of live-cell movies of tubular lysosomes undergoing elongation (top panel-across), fusion (middle panel) and branching (bottom panel). Arrowheads indicate TLs undergoing each event. Numbers represent time points in seconds. Scale bars, $5 \mu \mathrm{m}$. 
Figure 3.1
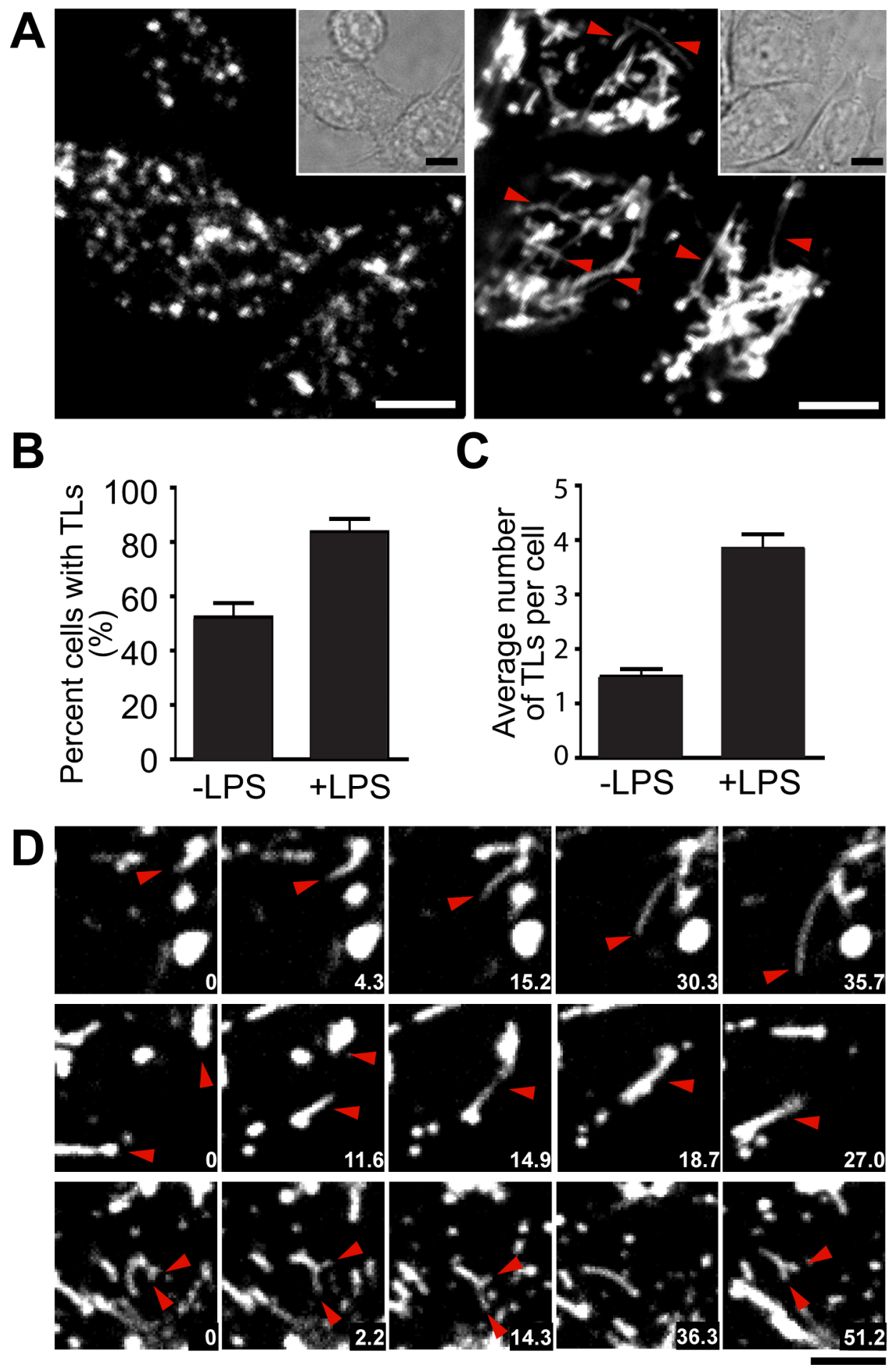
Figure 3.2: Dynein and Kinesin I are Required for Lysosome Tubulation. (A-C) Lysosomes of LPS-stimulated RAW cells were labeled with fluorescent dextrans (left). Arrowheads indicate TLs. Scale bars, $10 \mu \mathrm{m}$. (A) LPS-treated cells before (left) and after (right) treatment with $20 \mu \mathrm{M}$ nocodazole for $0.5 \mathrm{~h}$. (B) Cells expressing KIF5DN-RFP (right, outlined on left) show abated TL formation compared to surrounding untransfected cells. (C) Cells expressing dynamitin-GFP (right, outlined on left) show reduced number of TLs compared to untransfected cells. (D) Quantification of number of TLs in cells inhibited for kinesin and dynein. Data are mean \pm SEM normalized to untransfected control cells within the same experiment $(n=4$ experiments, 30-60 cells per condition per experiment; $p<0.0001$ for both experimental conditions). 
Figure 3.2

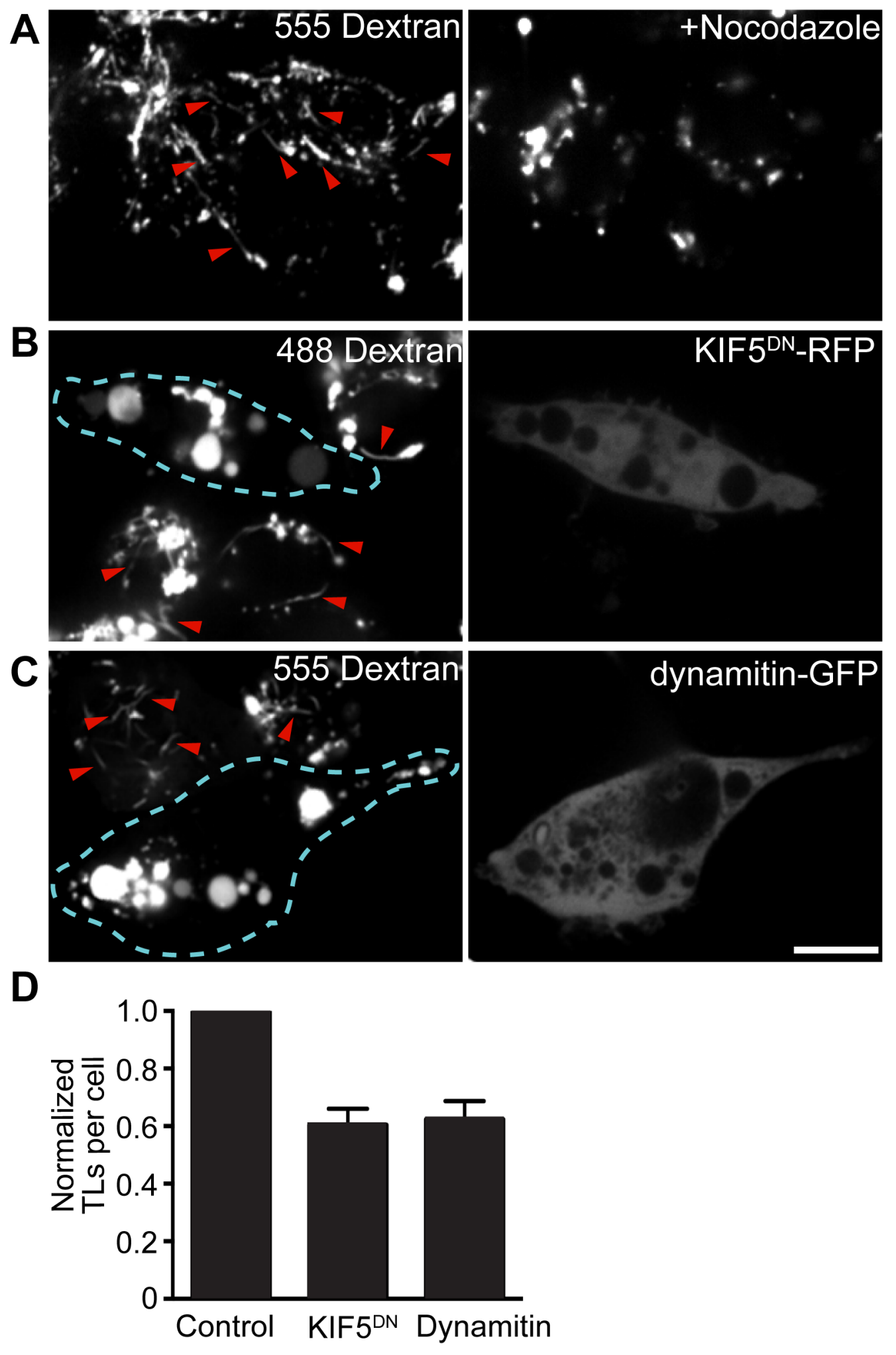


Figure 3.3: Rab7 is Required for Lysosome Tubulaton. (A-C) Lysosomes of LPSstimulated RAW cells labeled with Alexa-555-dextran (left) and expressing GFPtagged Rab7 probes (right). Arrowheads indicate TLs. Scale bars, $10 \mu \mathrm{m}$. (A) Cells expressing GFP-Rab7 (right, outlined on left) show that GFP-Rab7 colocalized with dextran labeled TLs (left). (B) Cells expressing GFP-RILP-c33 (right, outlines on left), a probe for active Rab7, indicate that TLs display GTP-Rab7. (C) Cells expressing GFP-Rab7222N (right, outlined on left) experienced a strong decline in lysosome tubulation compared to control GFP-Rab7 transfected cells. (D) Quantification of lysosome tubulation. Data are mean number of TLs per cell \pm SEM in GFP-Rab7 ${ }^{\text {T22N }}$ transfectants normalized to cells transfected with GFP-Rab7 ( $\mathrm{n}=3$ experiments, with $>30$ cells per condition per experiment; $p<0.0001$ ). 
Figure 3.3

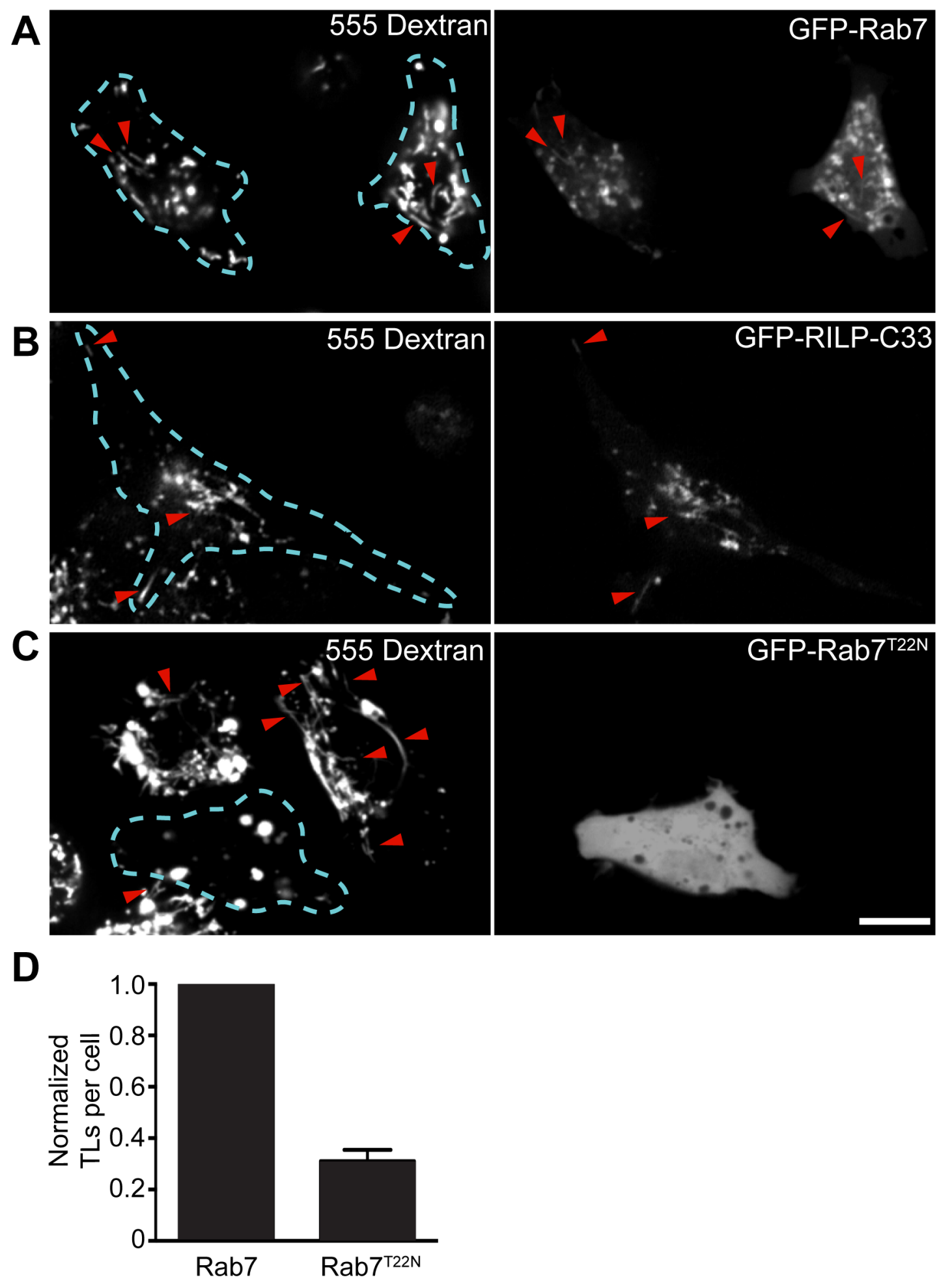


Figure 3.4: RILP is Involved in Lysosome Tubulation. (A) Lysosomes of LPSsimulated, GFP-RILP-expressing (right) RAW cells were labeled with TMR dextran (left). GFP-RILP localized to existing TLs, as indicated by arrowheads. Scale bars, 10 $\mu \mathrm{m}$. (B) Quantification of lysosome tubulation in cells overexpressing GFP-RILP, normalized to untransfected control cells. Data are mean \pm SEM $(n=3$ experiments, $>20$ cells/condition/experiment; $\mathrm{p}<0.0003$ ). (C) Quantification of RILP silencing using two different siRNA oligos (siRNA oligo 11 and 12). Data are mean \pm SEM, normalized to the corresponding scrambled control ( $n=3$ experiments; $p<0.005$ ). (D) Quantification of lysosome tubulation in RILP-silenced cells, normalized to corresponding scrambled control. Data are mean number of TLs per cell \pm SEM ( $n=3$ experiments for each oligo with $>30$ cells quantified per condition, per experiment; $\mathrm{p}<0.0001$ for each treatment). 
Figure 3.4
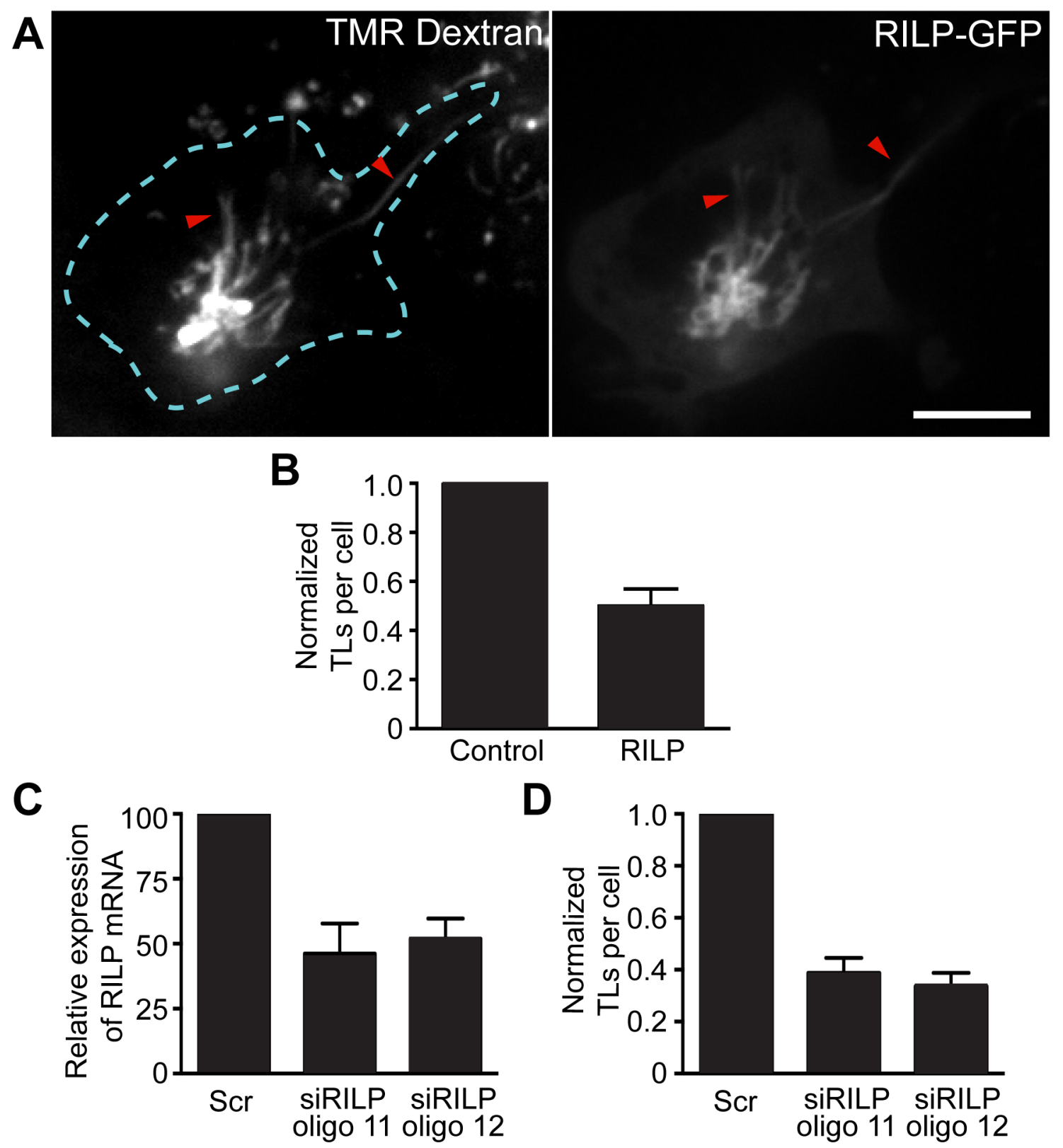
Figure 3.5: FYCO1 is Involved in Lysosome Tubulation. (A and B) Lysosomes of LPSstimulated, mCherry-FYCO1 transfected cells (right, outlined on left) RAW cells were labeled with Alexa-488-dextran (left). Tubular lysosomes are highlighted by arrowheads. Scale bars, $10 \mu \mathrm{m}$. (A) Cells overexpressing FYC01 show a reduced number of TLs relative to surrounding untransfected cells. (B) mCherry-FYCO1 labeled TLs. (C) Quantification of lysosome tubulation in cells overexpressing mCherry-FYC01, normalized to control untransfected cells ( $\mathrm{n}=3$ experiments, 20-40 cells quantified per experiment, per condition; $\mathrm{p}$ <0.0001). (D) Quantification of FYC01 expression by qRT-PCR following silencing with SMARTpool siRNA oligo mix or two individual siRNA oligos (9 and 11). Data are mean \pm SEM, normalized to respective scrambled control. (For SMARTpool mix: $n=4$ experiments, $\mathrm{p}<0.0001$; for oligos 9 and 11: $\mathrm{n}=3$ experiments each, $\mathrm{p}<0.006$ ). (E) Quantification of lysosome tubulation in FYCO1-silenced cells normalized to corresponding scrambled oligos. Data are mean number of TLs per cell \pm SEM. (For the SMARTpool mix: $n=4$ experiments, with $>40$ cells quantified per condition per experiment; for oligos 9 and 11: $n=3$, with $>30$ cells counted per condition per experiment, $p<0.0001$ for all conditions). 
Figure 3.5

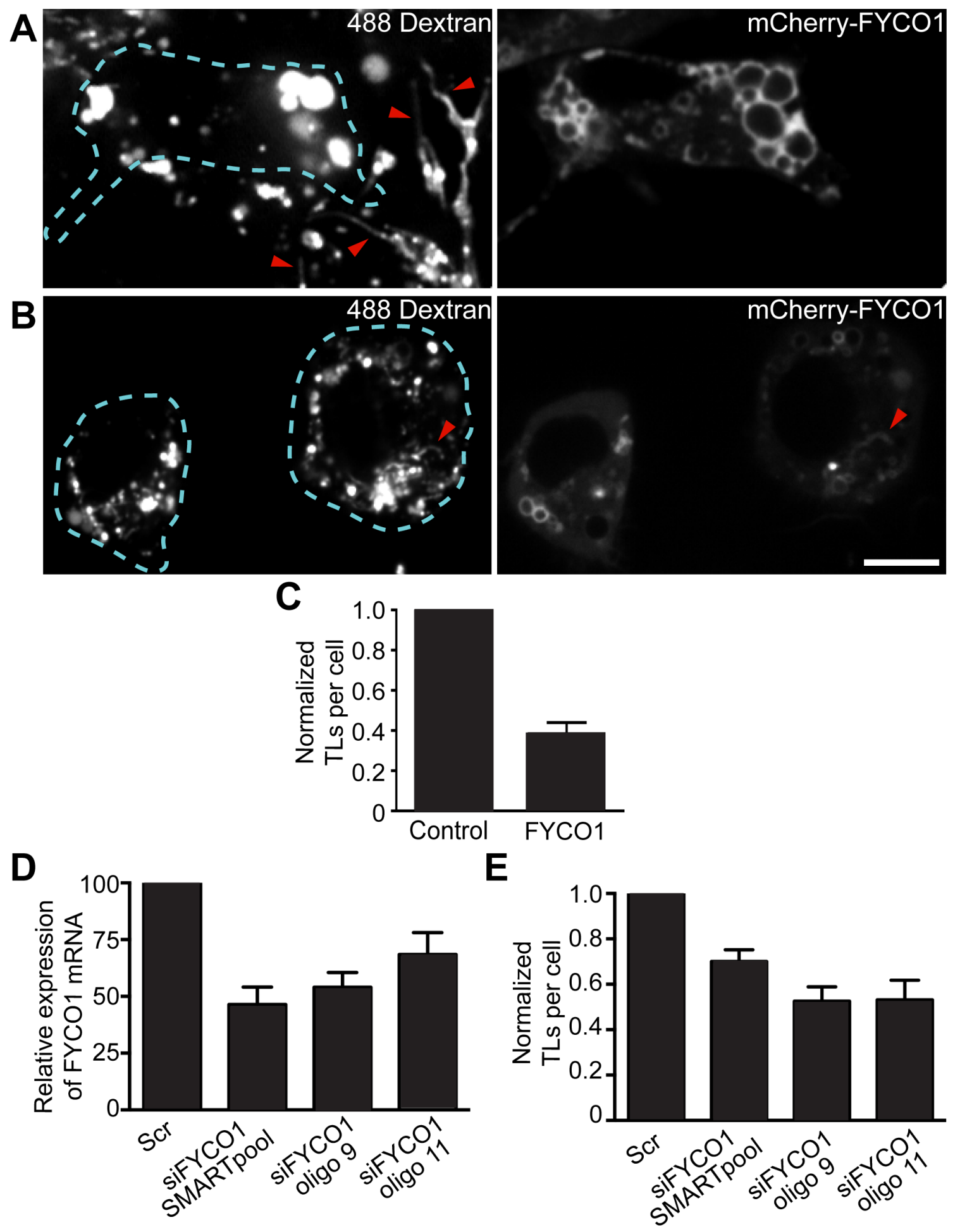


Figure 3.6: Arl8b and SKIP are Required for Lysosome Tubulation. (A and B) Lysosomes of LPS-stimulated RAW cells transfected with Arl8b-GFP probes (right, outlined in left) were labeled with Alexa-555-dextran (left). Arrowheads indicate TLs. Scale bars, $10 \mu \mathrm{m}$. (A) TLs are labeled with Arl8b-GFP. (B) Cells expressing Arl8bT24N-GFP are strongly impaired in their ability to for TLs compared to surrounding untransfected cells. (C) Quantification of lysosome tubulation in Arl8bT24N-GFP expressing cells, normalized to cells expressing the wild-type Arl8b. Data are mean number of TLs per cell \pm SEM ( $n=3$ experiments, with $>15$ cells quantified per condition per experiment; p<0.0001). (D) Western blot showing knockdown of Arl8b in Arl8b-silenced RAW cells using the SMARTpool mix. The 'RAW lysate' and 'Scrambled' lanes show Arl8b expression in whole cell lysates of untreated RAW cells and RAW cells treated with scrambled siRNA, respectively. Arl8b was probed using anti-Arl8b antibodies that were a generous gift from Dr. Michael Brenner (Harvard). GAPDH expression was used as a loading control and was detected using anti-GAPDH antibodies. (E) Quantification of Arl8b silencing using two different siRNA oligos (oligos 10 and 12). Data are mean \pm SEM, normalized to scrambled control ( $\mathrm{n}=3$ experiments, $\mathrm{p}<0.0001$ for each oligo). (F) Quantification of TL formation in Arl8b-silenced cells. Data are mean number of TLs per cell \pm SEM, normalized to the corresponding scrambled control oligos $(n=3$ experiments, with $>20$ cells quantified per condition per experiment; $p<0.0001$ for each condition. (G) Quantification of SKIP silencing using SMARTpool mix or two individual oligos (5 and 7). Data are mean \pm SEM, normalized to respective scrambled controls $(\mathrm{n}=3$ experiments; $\mathrm{p}<0.0040$ for each condition. Quantification of TL formation in SKIP-silenced cells. Data are mean number of TLs per cell \pm SEM, normalized to control cells treated with scrambled siRNA $(n=3$ experiments, with $>20$ cells counted per condition per experiment; $\mathrm{p}<0.0001$ for each condition). 
Figure 3.6

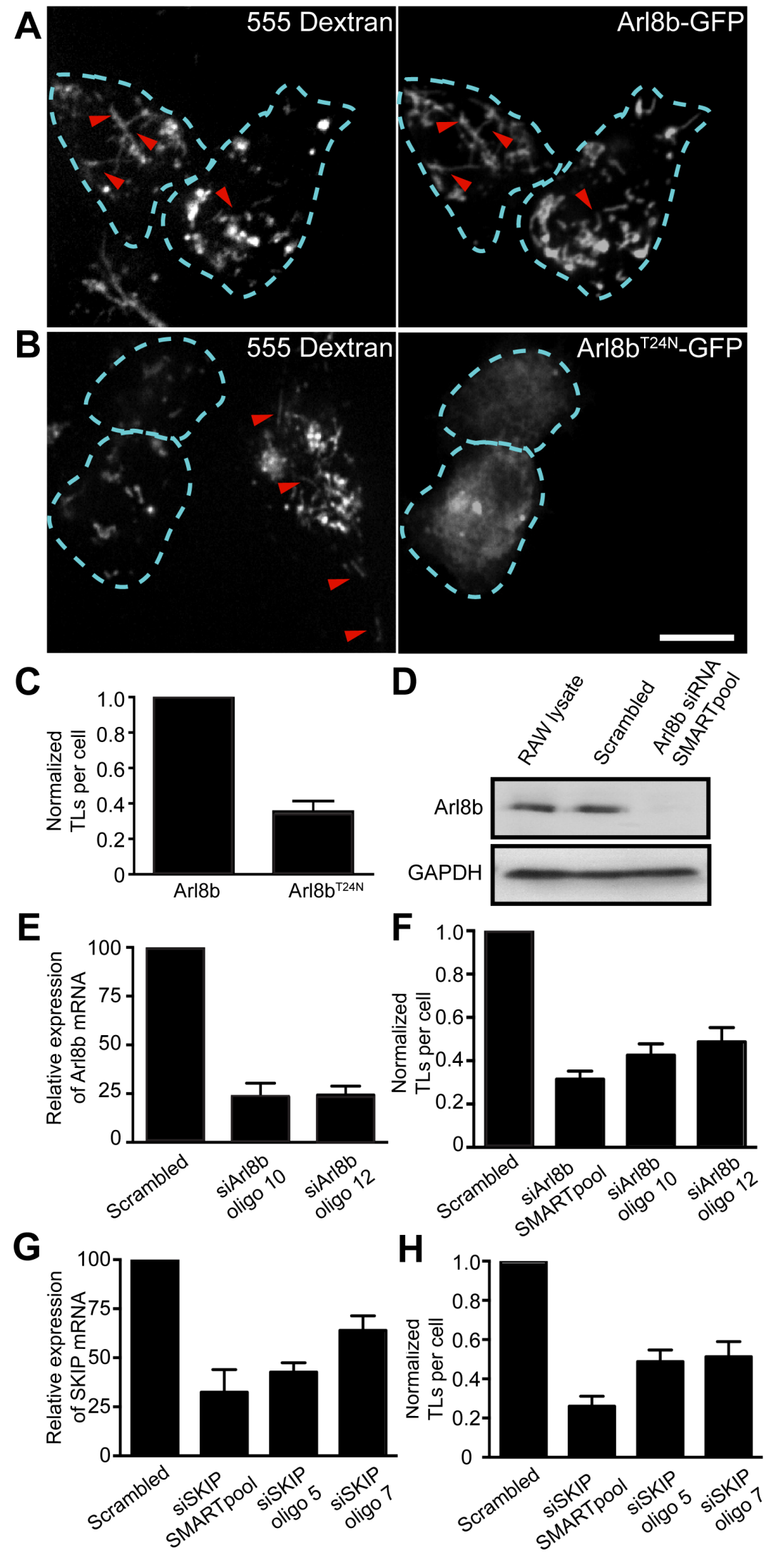


Figure 3.7: Tubular Lysosomes are Highly Motile. (A and B) Motility tracks of randomly selected lysosomes in LPS-treated cells, where individual punctate lysosomes (A) and TLs (B) are represented by different colours. All lysosomes were tracked for 1 min each and tracks were overlayed to show total distance traveled by each lysosome. (C) The displacement distance between starting and end points after 1 min of observation for punctate and TLs in LPS-treated cells, data are means \pm SEM and are based on $>30$ randomely chosen lysosomes/TLs within 15 different cells from three independent experiments. Means were compared using the Student's t-test with a $\mathrm{p}<0.0001$. (D) Total track lengths after 1 min of observation for punctate and TLs in LPS-treated cells, data are means \pm SEM and are based on $>30$ randomly chosen lysosomes/TLs within 15 different cells from three different experiments. Means were compared using the Student's t-test with a $p<0.0009$. (E) Quantification of lysosome mean velocities for punctate and TLs in LPS and nonLPS-treated cells. Data are mean \pm SEM and are based on $>30$ randomly chosen lysosomes/TLs among 15 different cells from three independent experiments. Mean velocities of puctate lysosomes and TLs in LPS-treated cells were tested against those in non-LPS-treated cells using Student's t-test with p-values of 0.0097 and 0.0006 , respectively. 
Figure 3.7
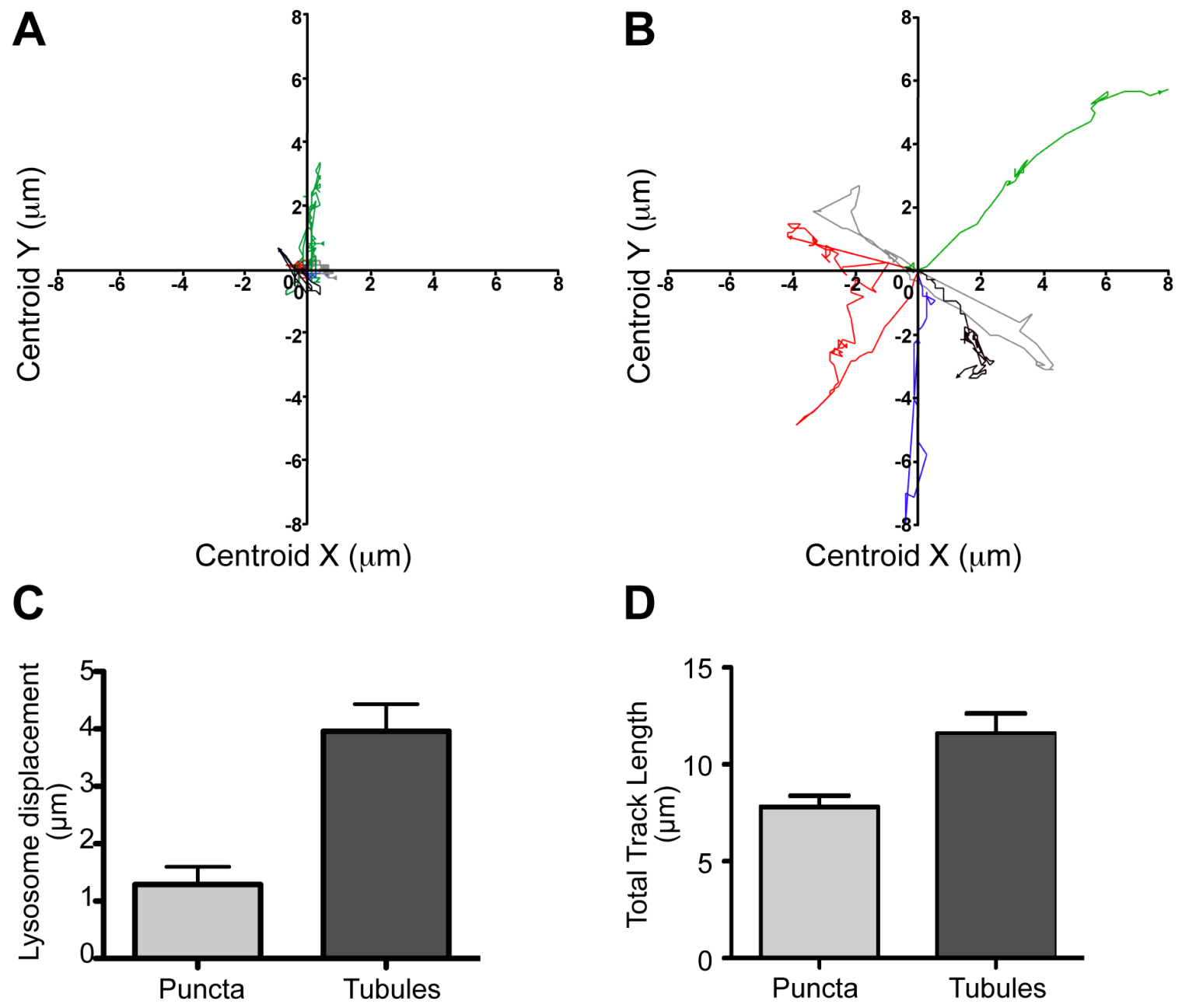

E

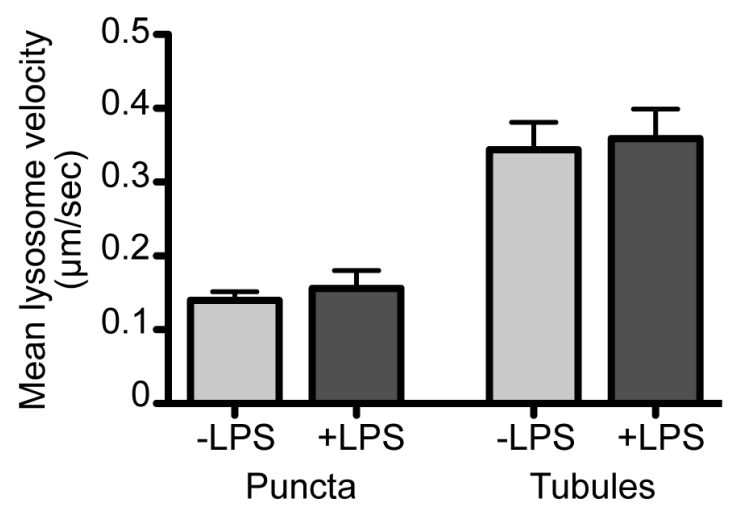


Figure 3.8: Schematic of TLR4 Signaling Pathways and Compounds that Selectively Inhibit Them. Three major signaling pathways are known to eminate downstream of the LPS receptor, TLR4. These include the PI3K, which is likely recruited to TLR4 via the adapter MyD88 (Laird et al., 2009). The MyD88-IRAK-TRAF6 pathway, which ultimately leads to cytokine production by inducing nuclear translocation of the transcription factor NFKB. Finally, the TRAM/TRIF-TBK1-IRF3 pathway is also known to be activated downstream of TLR4 and results in production of proinflammatory mediators through transcriptional control. To inhibit each pathway independently, we applied the compounds LY294002, IRAK1/4 inhibitor and MRT67007 to inhibit PI3K, IRAK1/4 and TBK1, respectively. In addition, PepinhMYD, a MyD88-inhibitory peptide was used to block MyD88 signaling. 
Figure 3.8

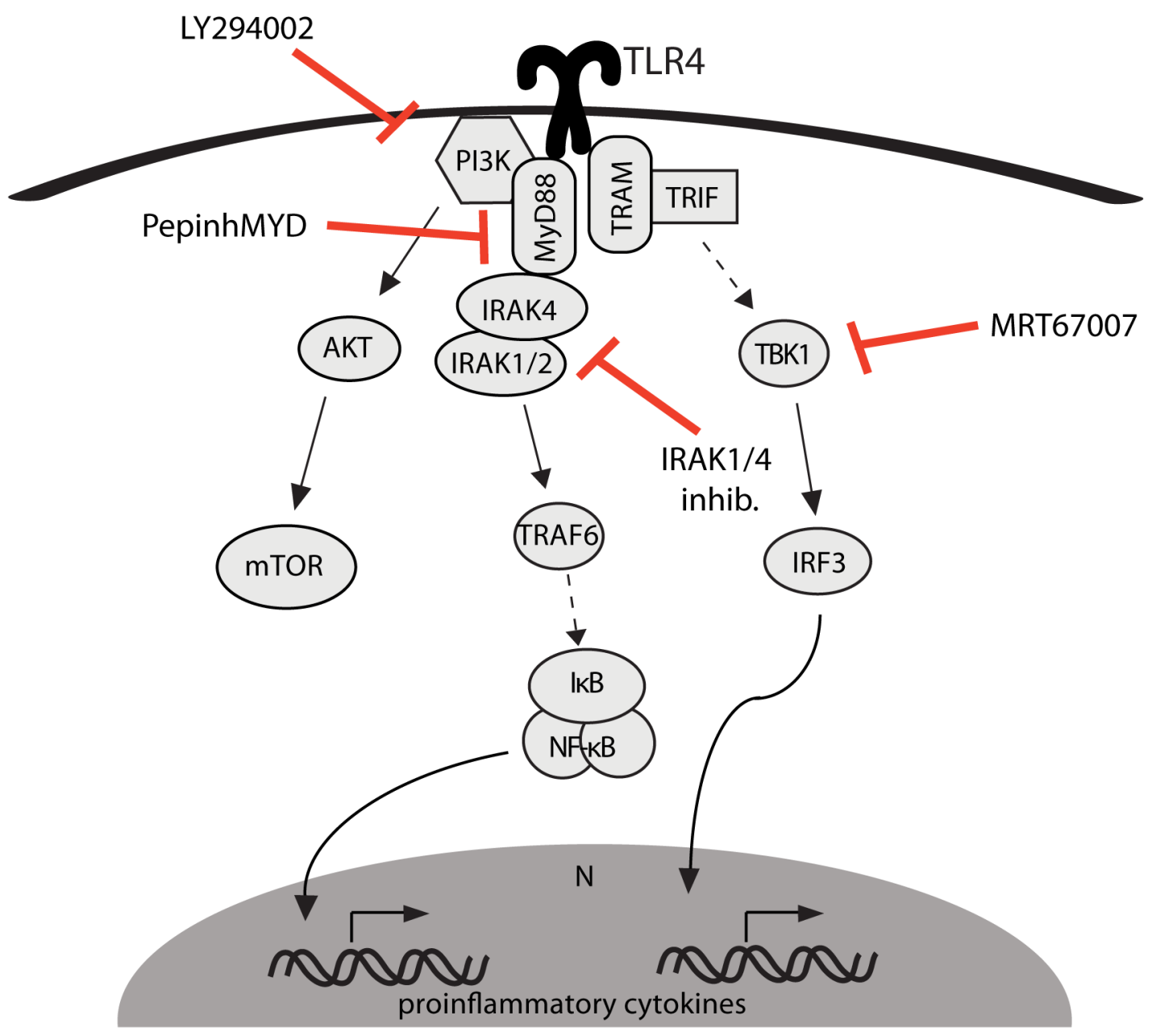

80 
Figure 3.9: MyD88 and PI3K are Required for LPS-Induced Lysosome Tubulation in Macrophages. (A) Lysosomes of RAW 264.7 macrophages labeled with Alexa555 dextran. Cells were either treated with the vehicle dimethyl sulfoxide (DMSO) alone as a control, or pre-treated for 20 min with DMSO, $2 \mu \mathrm{M}$ TBK1 inhibitor (MRT67307), $5 \mu \mathrm{M}$ IRAK1/4 inhibitor, $1 \mu \mathrm{M}$ PI3K inhibitor (LY294002) or $1 \mu \mathrm{M}$ Class I PI3K inhibitor (ZSTK474) before stimulating with $100 \mathrm{ng} / \mathrm{mL}$ LPS for $2 \mathrm{~h}$ to induce lysosome tubulation. Alternatively, cells were incubated with Pepinh-ctrl (control peptide) or Pepinh-MYD (MyD88-inhibitory peptide) for $3 \mathrm{~h}$ before stimulating with LPS. LPS induces extensive tubulation but not in cells inhibited for MyD88 and PI3Ks. Dashed lines outline individual cells. Red arrowheads denote individual lysosome tubules. Scale bar $=10 \mu \mathrm{m}$. (B) Quantification of lysosome tubulation under conditions described in $A$. Shown are the mean \pm SEM of three independent experiments based on 25-30 cells per condition per experiment. Data was statistically analysed using a one-way ANOVA, followed by Tukey's post-hoc test. An asterisk $(*)$ indicates a significant difference $(p<0.001)$ between LPS alone and cells pre-treated with the MyD88 inhibitory peptide and the PI3Ks inhibitors. 
Figure 3.9
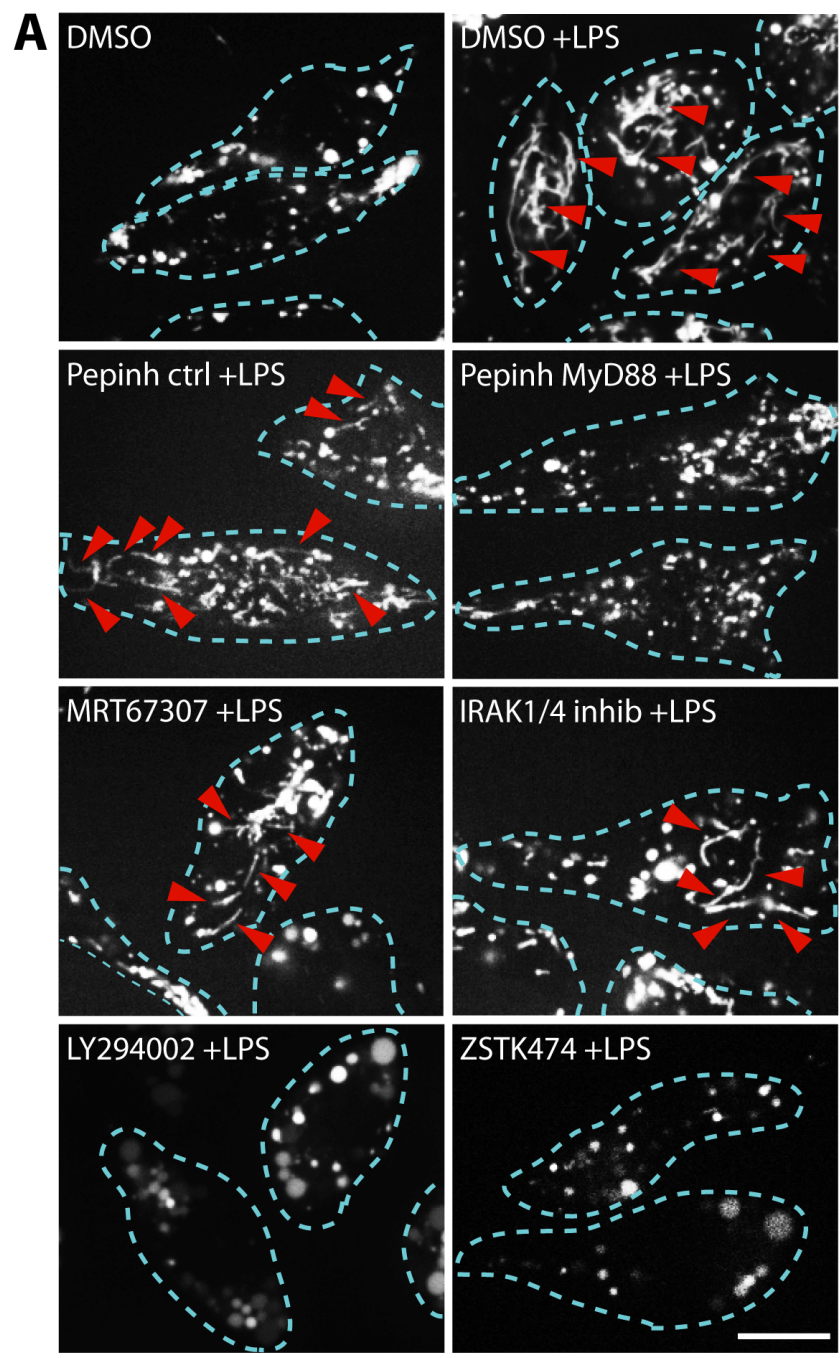

B

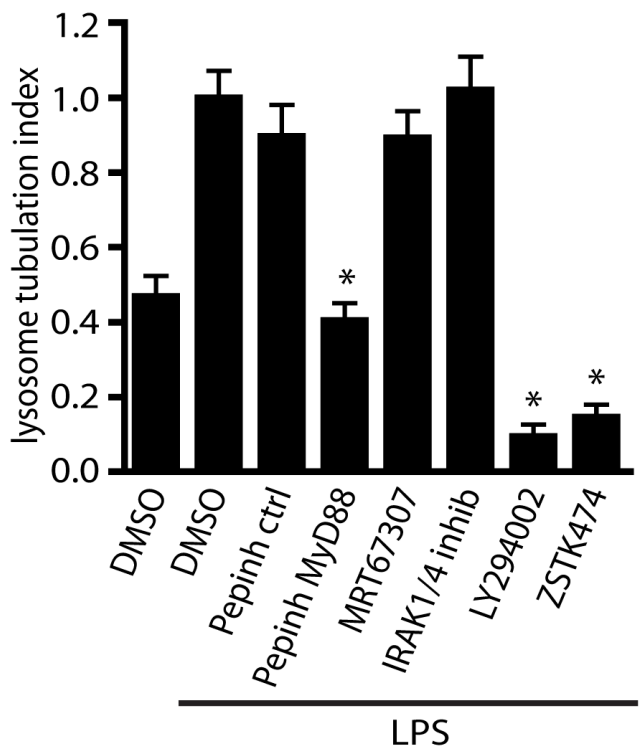


Figure 3.10: The Canonical PI3K-Akt Pathway Regulates LPS-Induced Lysosome Tubulation in Macrophages. (A) Western blots of whole cell lysates from RAW cells treated as indicated across lanes. LPS increases the levels of activated Akt (pAkt) in a PI3K-dependent manner, but independently of IRAK1/4 and TBK1 activity. Total Akt was probed for as loading control. (B) Lysosomes of RAW 264.7 macrophages labeled with Alexa555 dextran. Cells were treated with vehicle alone (DMSO) or pre-treated for 20 min with DMSO, or $5 \mu \mathrm{M}$ Akt inhibitor (Akti) before $2 \mathrm{~h}$ LPS stimulation. Extensive lysosomal tubules appear upon LPS stimulation, but not in cells inhibited for Akt. Dashed lines outline individual cells. Red arrowheads denote individual TLs. Scale bar $=10 \mu \mathrm{m}$. (C) Quantification of lysosome tubulation under conditions described in $B$. Shown are the mean \pm SEM of three independent experiments based on 25-30 cells per condition per experiment. Data was statistically analysed using a one-way ANOVA, followed by Tukey's post-hoc test. An asterisk $\left(^{*}\right)$ indicates a significant difference $(p<0.0001)$ between LPS alone and cells inhibited for Akt. 
Figure 3.10

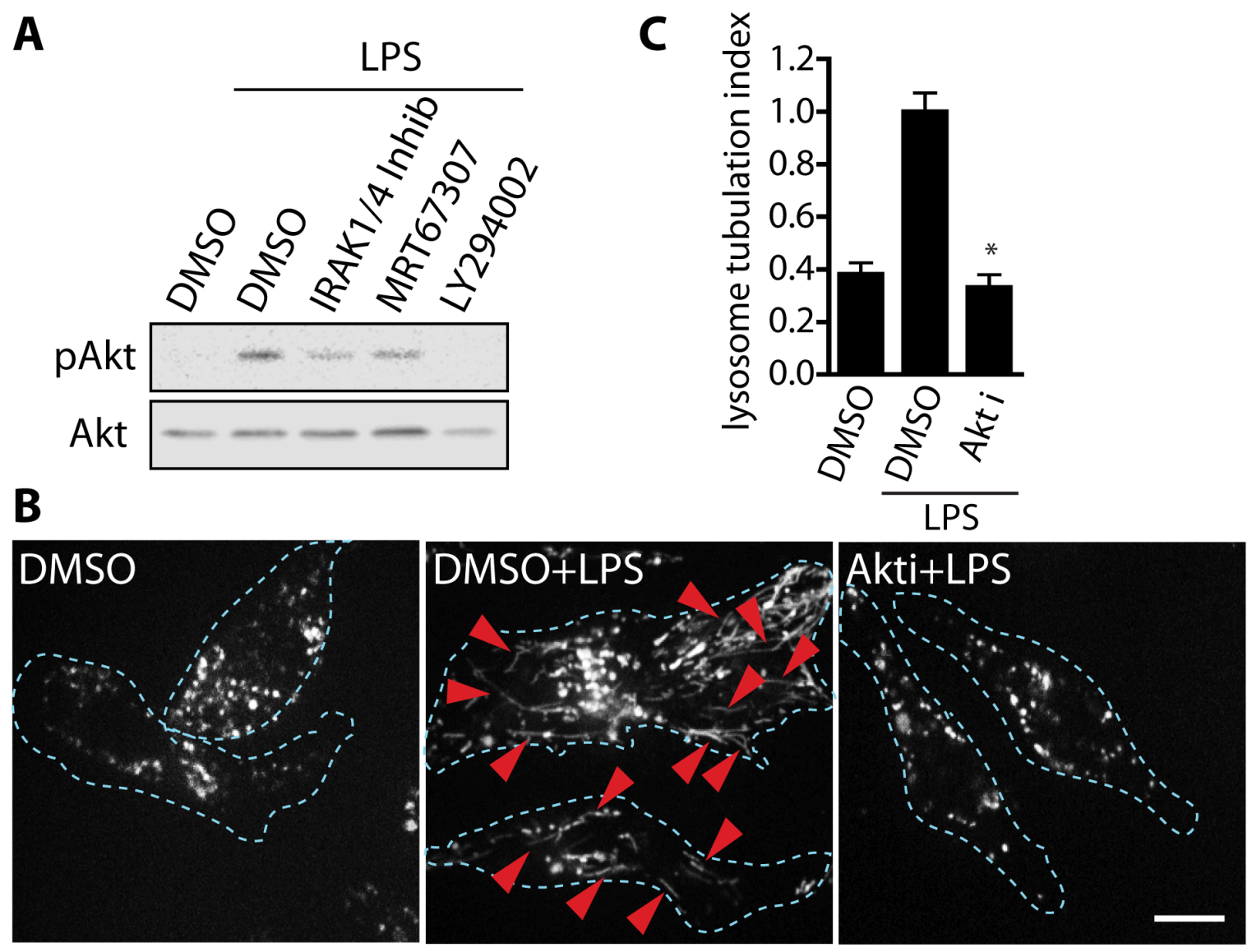


Figure 3.11: mTOR is Activated by LPS and is Required for Lysosome Tubulation in Macrophages. (A) Western blots of whole cell lysates from RAW cells treated as indicated across lanes. LPS treatment for 1 and $2 \mathrm{~h}$ enhances S6K phosphorylation (pS6K) relative to non-LPS treated cells and this is suppressed by torin1. Total S6K was probed for as a loading control. (B) LPS-dependent increase in pS6K levels is dependent on PI3K but independent of IRAK1/4 and TBK activity. (C) Lysosomes of RAW 264.7 macrophages labeled with Alexa555 dextran. Cells were treated with vehicle alone (DMSO) or pre-treated for 20 min with DMSO, or $100 \mathrm{nM}$ torin1 before $2 \mathrm{~h}$ LPS stimulation. Extensive lysosomal tubules appear upon LPS stimulation, but not in cells inhibited for mTOR. Dashed lines outline individual cells. Red arrowheads denote individual TLs. Scale bar $=10 \mu \mathrm{m}$. (D) Quantification of lysosome tubulation under conditions described in $C$. (E) Lysosomes labeled with Alexa555 dextran in LPS-treated cells electroporated with non-targeting pool (siNTP) or with mTOR-targeting siRNA oligonucleotides (simTOR). (F) Quantification of lysosome tubulation under conditions described in E. Data are mean \pm SEM of four independent experiments containing 25-30 cells per condition. (G) Treatment of RAW cells with the AMPK activator A769662 blocks TL formation. (H) Quantification of lysosome tubulation under conditions described in $G$. Data are mean \pm SEM of three independent experiments containing 25-30 cells per sample. For $D$, data were statistically tested using one-way ANOVA test, followed by Tukey's test. For $G$ and $H$, data were tested with a paired Student's t-test, ${ }^{*} p<0.0001$. Arrowheads indicate TLs in micrographs. Scale bars $=10 \mu \mathrm{m}$. 
Figure 3.11

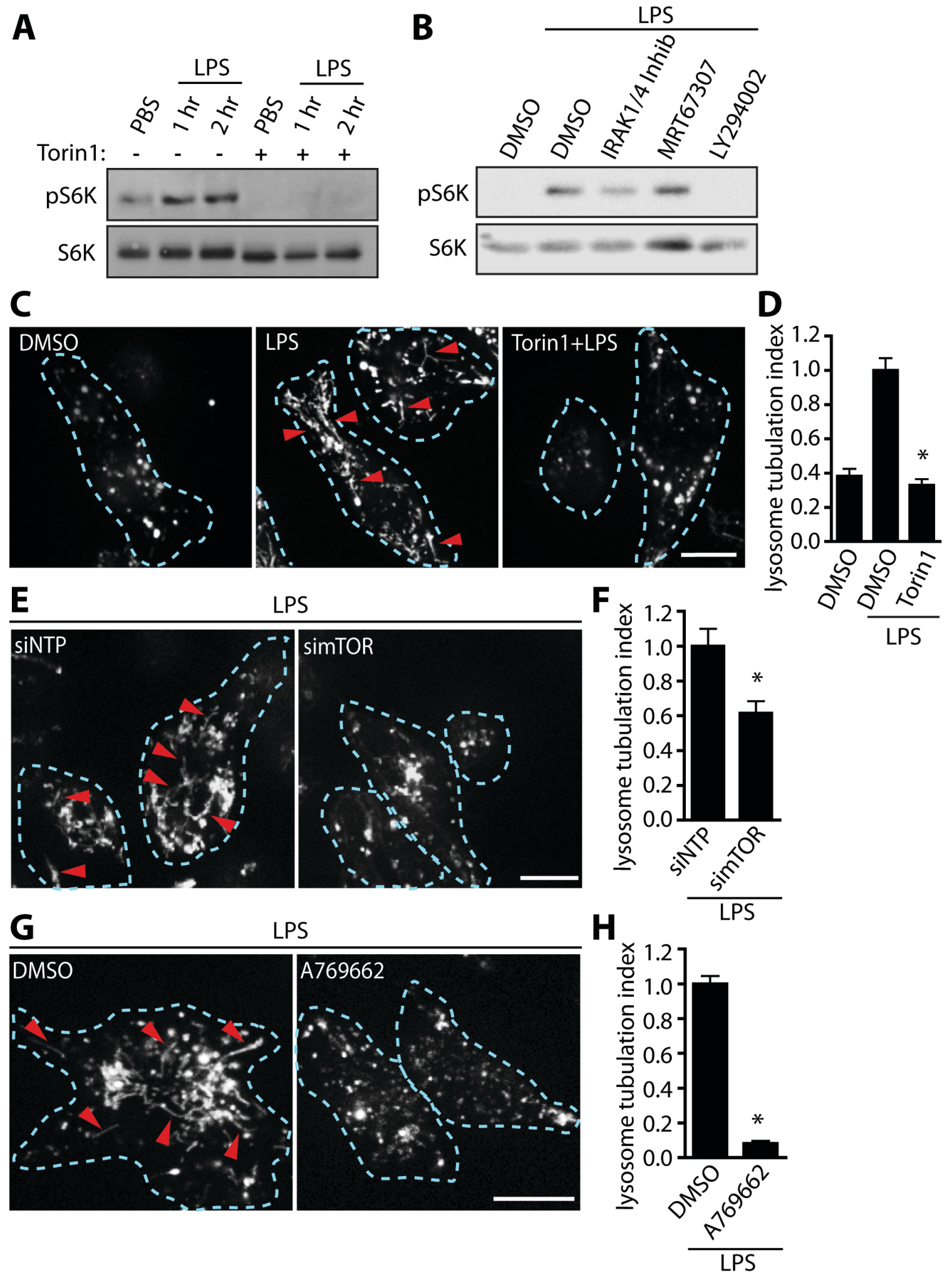


Figure 3.12: The Akt-mTOR Pathway is Activated Early and Maintained Under LPS Signaling and Coincides with Lysosome Tubulation. (A) Western blots of RAW whole-cell lysates following LPS stimulation for the time points indicated across top (in minutes). LPS stimulation caused phosphorylation of Akt and S6K as early as 15 minutes, increasing at $30 \mathrm{~min}$ and remaining for at least $2 \mathrm{~h}$. (B) Lysosomes of RAW 264.7 macrophages labeled with Alexa555 dextran. Cells were treated with LPS and live-cell imaging was carried out at the time points indicated (in minutes). Dashed lines outline individual cells. Red arrowheads denote individual TLs. Scale bar $=10$ $\mu \mathrm{m}$. (C) Quantification of lysosome tubulation under conditions described in $B$. Data was statistically analyzed using a one-way ANOVA, followed by Tukey's posthoc test. An asterisk $\left(^{*}\right)$ indicates a significant difference from 0 min condition $(p<$ $0.05)$. 
Figure 3.12
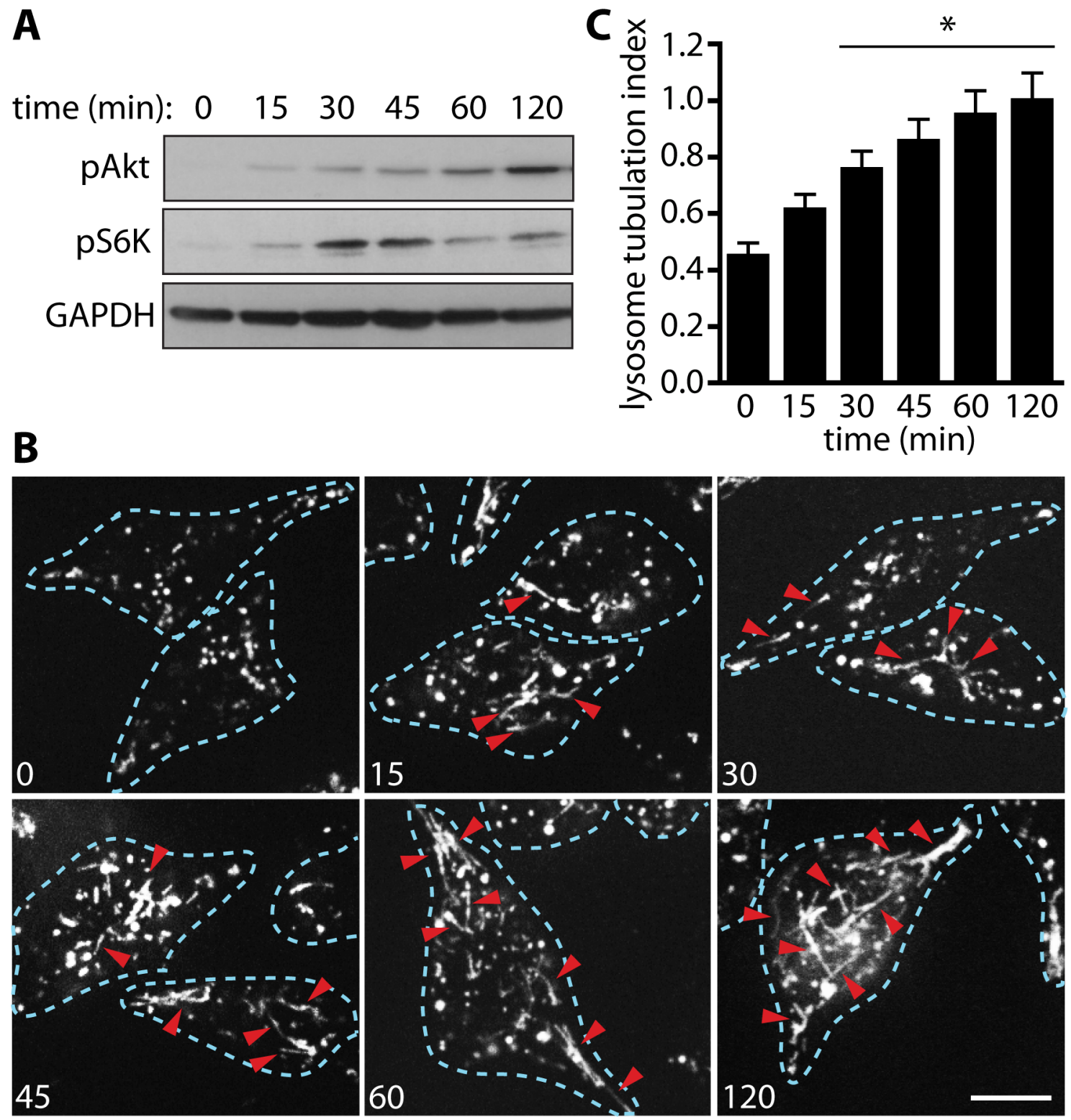
Figure 3.13: mTOR is Required for MIIC Tubulation in Primary Dendritic Cells. (A) LPS-stimulated BMDCs from MHC-II-GFP mice labeled with Alexa555-dextran. Merge shows intracellular co-localization of MHC-II and dextran. Insets highlight lysosomal tubules. (B) Western blot of whole cell lysates from wild type BMDCs. LPS treatment for 1 and $2 \mathrm{~h}$ increases the levels of phospho-S6K in a torin1dependent manner indicating that LPS activates mTOR. Total S6K was probed for as loading control. (C) Lysosomes of wild type BMDCs labeled with Alexa555 dextran. Cells were treated with vehicle alone (DMSO) or pre-treated for 20 min with DMSO, $100 \mathrm{nM}$ rapamycin, or $100 \mathrm{nM}$ torin 1 followed by $100 \mathrm{ng} / \mathrm{mL}$ LPS treatment for 2 hours to induce lysosome tubulation. Both torin1 and rapamycin appeared to strongly reduce lysosome tubulation in BMDCs. (D) Quantification of lysosome tubulation under conditions described in $C$. Data are mean \pm SEM of three independent experiments containing 25-30 cells per sample per experiment. Scale bars, $10 \mu \mathrm{m}$. Data are mean \pm SEM of three independent experiments containing 25 30 cells per sample. Data was statistically analyzed using a one-way ANOVA, followed by Tukey's post-hoc test. An asterisk $\left({ }^{*}\right)$ indicates a significant difference ( $p$ $<0.0001$ ) between LPS alone and cells exposed to rapamycin and torin1. 
Figure 3.13

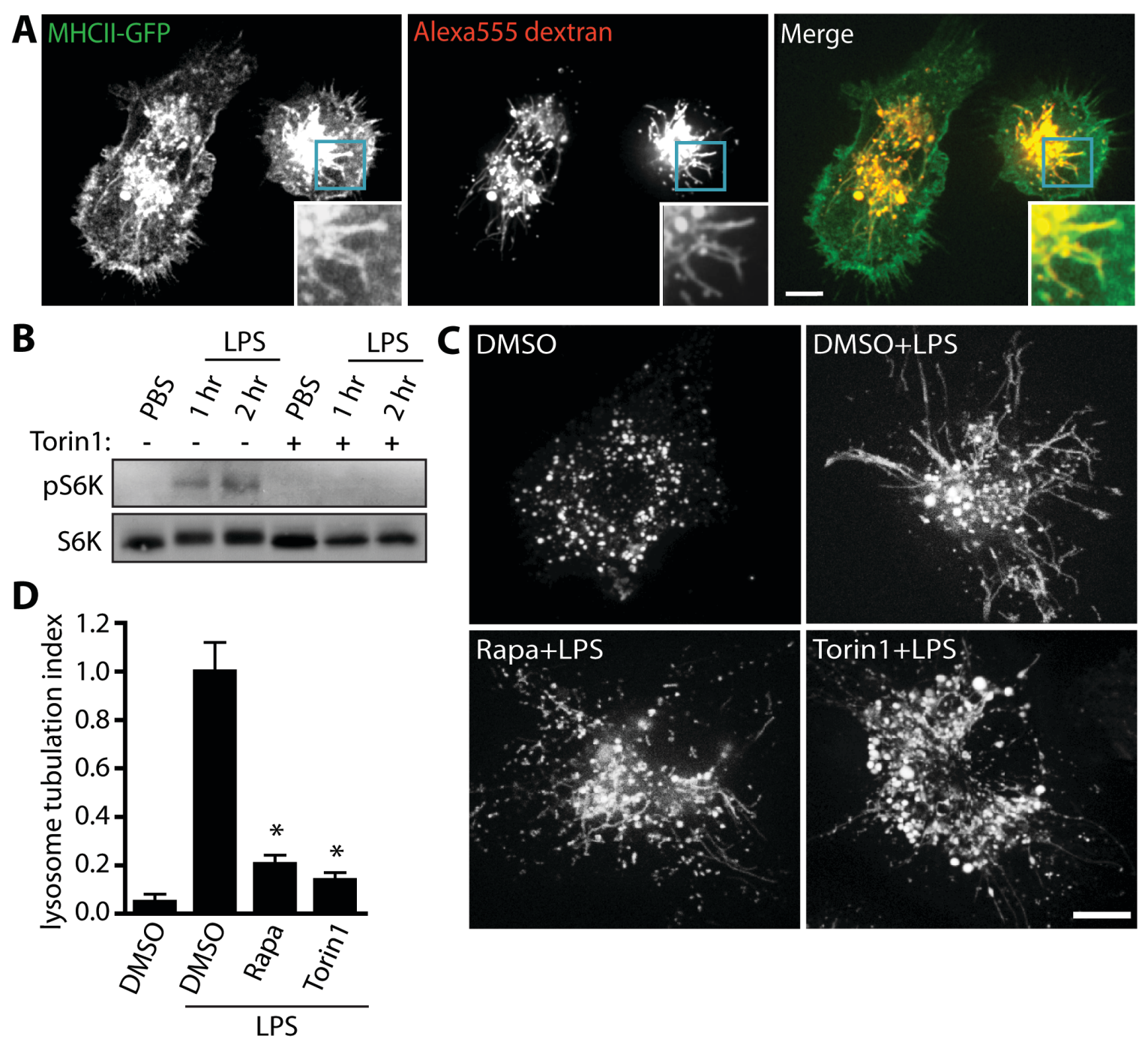


Figure 3.14: Torin1 Blocks Lysosome Tubulation in a Manner Independent of Autophagy. (A) Relative mRNA expression of ULK1 in RAW cells upon siRNA mediated silencing of ULK1 compared to control cells treated with siNTP. Data is mean \pm SEM of 3 independent experiments. (B) Immunofluorescence staining of RAW cells for LC3 in control (siNTP) and ULK1-silenced (siULK1) conditions. Note the increased punctate staining of LC3 in siNTP condition with $2 \mathrm{~h}$ of $200 \mathrm{nM}$ torin1, indicating autophagy induction, and lack of LC3 puncta in ULK1-silenced cells under the same condition indicating a block in autophagy. (C) Quantification of LC3 puncta in $B$. Data are mean \pm SEM from 2 individual experiments where 15-20 cells were analyzed per condition per experiment. (D) Lysosomes of siNTP (top panel) or siULK1 (bottom panel) RAW 264.7 macrophages were labeled with Alexa555 dextran. Cells were treated either with vehicle (DMSO) alone or pre-treated for 20 min with DMSO or $200 \mathrm{nM}$ torin1 followed by $2 \mathrm{~h}$ LPS stimulation. Dashed lines outline individual cells. Red arrowheads denote individual TLs. (E) Quantification of lysosome tubulation in $D$. Data was statistically analyzed using a two-way ANOVA, followed by Tukey's post-hoc test. In $C$, an asterisk $(*)$ indicates significant difference compared to siNTP/-torin 1 condition $(p<0.0001)$. In $E$, an asterisk $\left({ }^{*}\right)$ indicates significant difference between DMSO and DMSO+LPS conditions. Scale bars $=10 \mu \mathrm{m}$. 
Figure 3.14
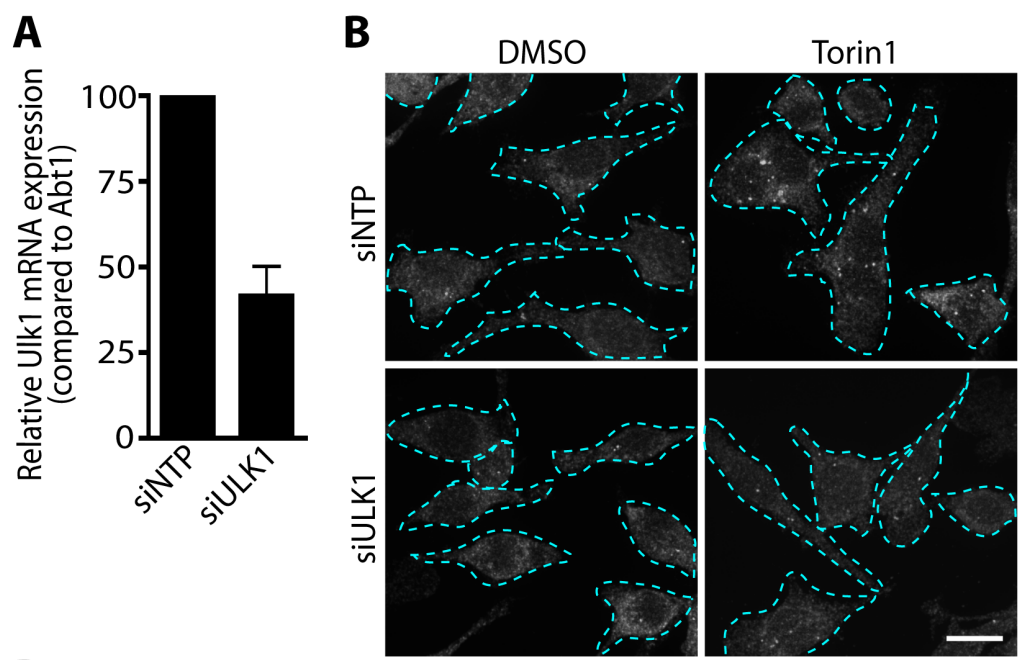

C

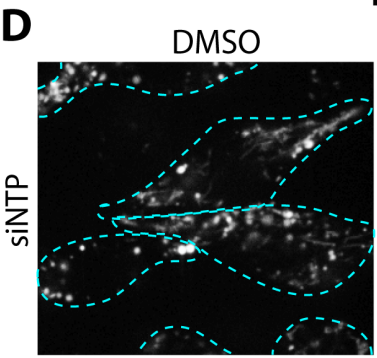

DMSO+LPS

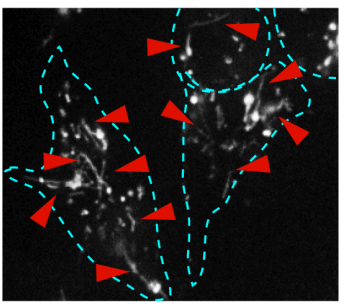

Torin 1+LPS
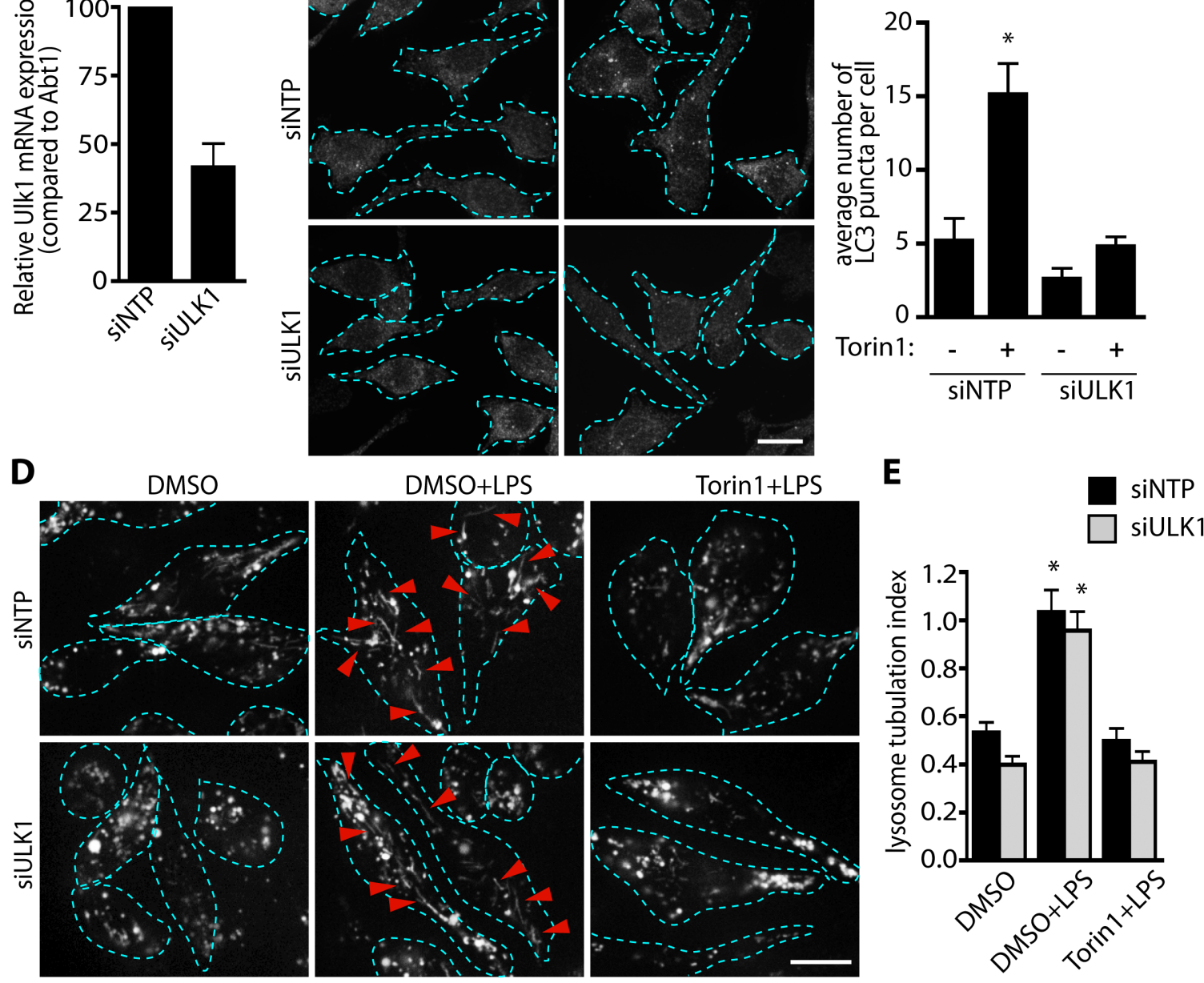

Torin 1:

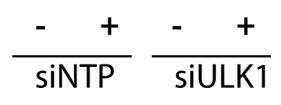


Figure 3.15: mTOR is Required for Anterograde Lysosomal Transport. (A) Lysosomes of RAW cells were pre-labeled with Alexa555-dextran and treated for $2 \mathrm{~h}$ with either DMSO (top row) or $200 \mathrm{nM}$ torin1 (bottom row). Cells were either left sitting in regular Ringer's medium ("resting"), treated with Acetate Ringer's for 20 min ("acetate") or treated with Acetate Ringer's for 20 min followed by regular Ringer's medium for 20 min ("recovery"). Scale bars $=10 \mu \mathrm{m}$. (B) Left: schematic of shell analysis in which three different regions (peripheral shell 1; middle shell 2, inner shell 3) were analyzed for percentage of lysosomes. Right: Quantification of the ratio of percent lysosomes in shell 1 to shell 3. Data are mean \pm SEM of tour independent experiments containing 10 cells per condition per sample. Data were statistically analyzed using a two-way ANOVA, followed by Tukey's post-hoc test. An asterisk $\left(^{*}\right)$ indicates significant difference between control acetate and torin1acetate treatments, ${ }^{*} p<0.05$. 
Figure 3.15
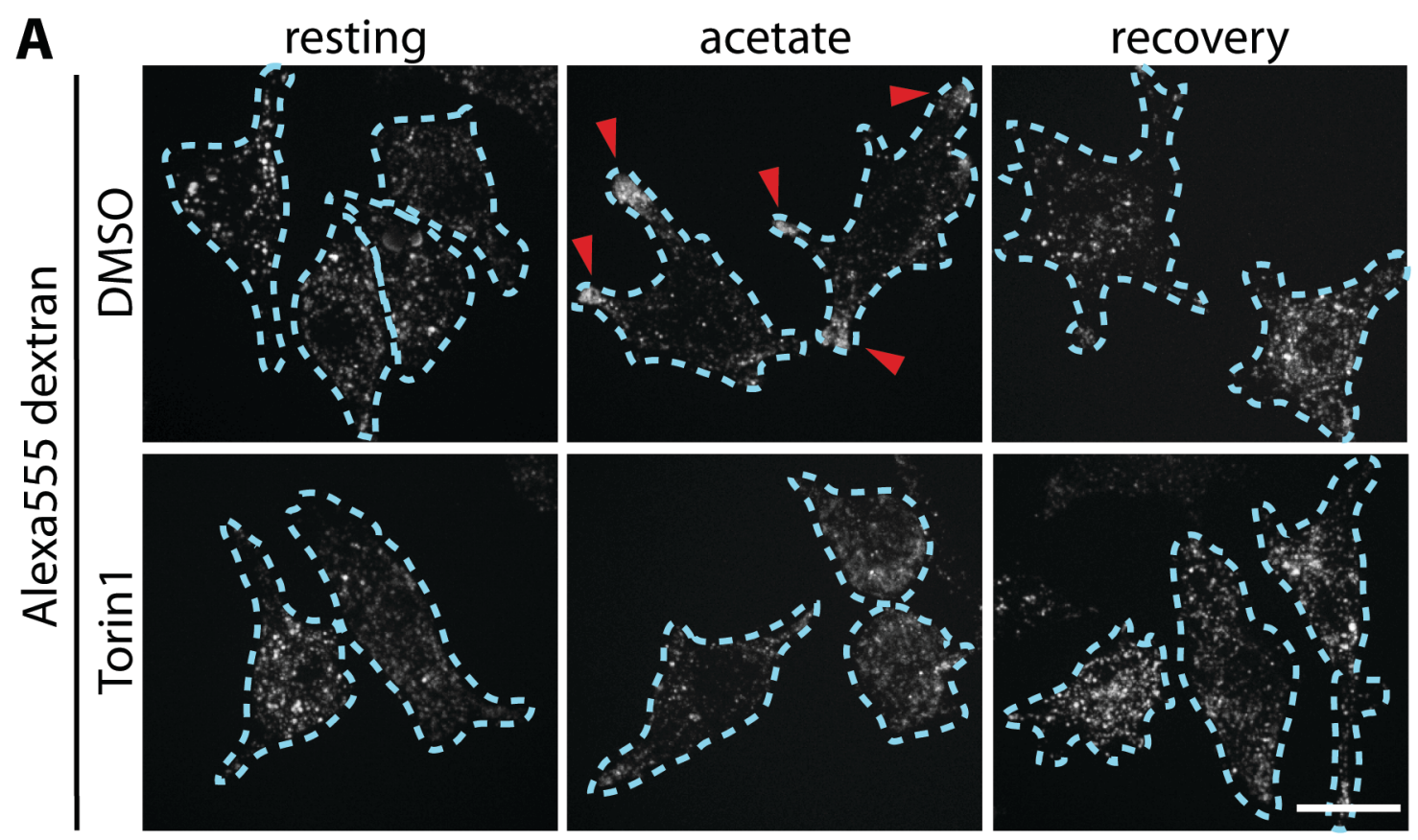

B

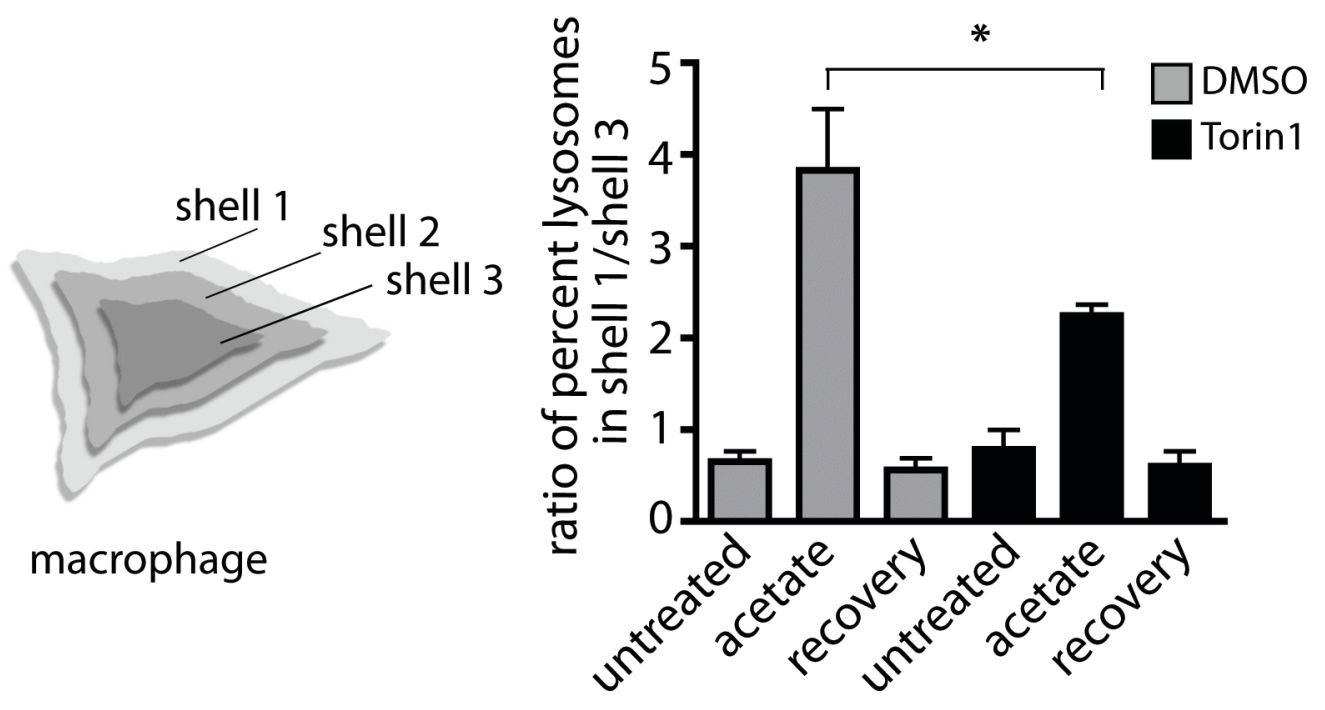


Figure 3.16: mTOR is Required for LPS-Induced Cell Surface Delivery of MHCII. (A) BMDCs from wild-type C57BL/6J mice were treated with DMSO for 5 hours, DMSO+LPS for 5 hours or pre-treated for 20 min with Torin1 followed by LPS+Torin 1 for 5 hours. Cells were stained live and gated on the DC-specific marker CD11c. This subset was analyzed for cell surface MHCII using anti-MHCII antibodies and flow cytometry. Data are mean \pm SEM of seven independent experiments, normalized to control (DMSO). 10,000 events were collected per condition. Data were statistically analyzed by one-way ANOVA and Tukey's post-hoc test. An asterisk $\left(^{*}\right)$ indicated significant difference between LPS and LPS+Torin1 treatments $\left({ }^{*} \mathrm{p}<0.0001\right)$. (B) Western blot of BMDC whole-cell lysates treated as described in (A) showing total MHCII levels using an anti-MHCII antibody graciously provided by Dr. Shin (UCSF) and GAPDH as loading control (top). (C) Quantification of three individual western blots under conditions described, normalized to GAPDH. 
Figure 3.16

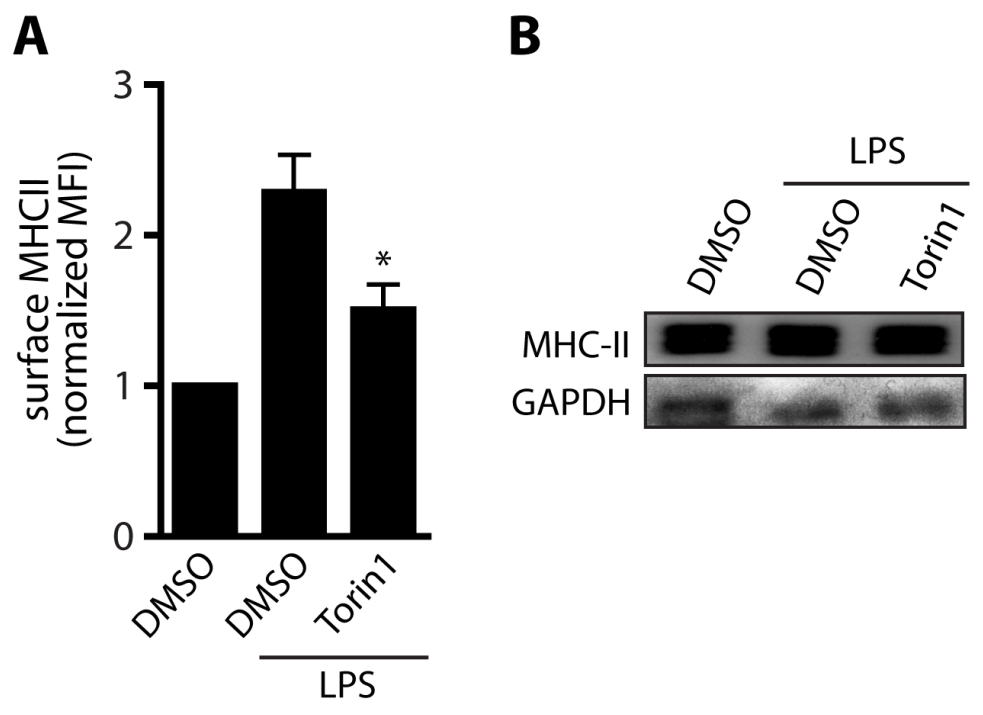

C

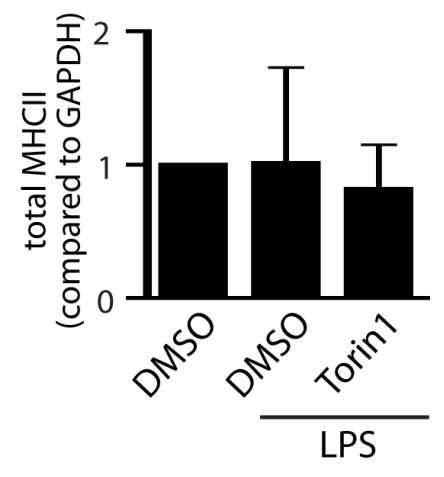


Figure 3.17: Model of Tubular Lysosome Biogenesis in Macrophages and Dendritic Cells. Activation of TLR4 with LPS engages the adaptor MyD88, which recruits PI3K leading to the activation of Akt and mTOR. mTOR likely modulates lysosome morphology by controlling microtubule-dependent motor activity of the organelle. In turn, microtubule motors link to lysosomes through Rab7 and Arl8b GTPases and their effectors RILP, FYCO1 and SKIP to allow for stretching of this organelle along microtubules. A functional consequence of inadequate mTOR signaling is not only lack of TL formation but also delivery of MHCII to the plasma membrane, which may be due to tubulation deficiency. Dashed lines indicate incompletely understood mechanisms. 
Figure 3.17

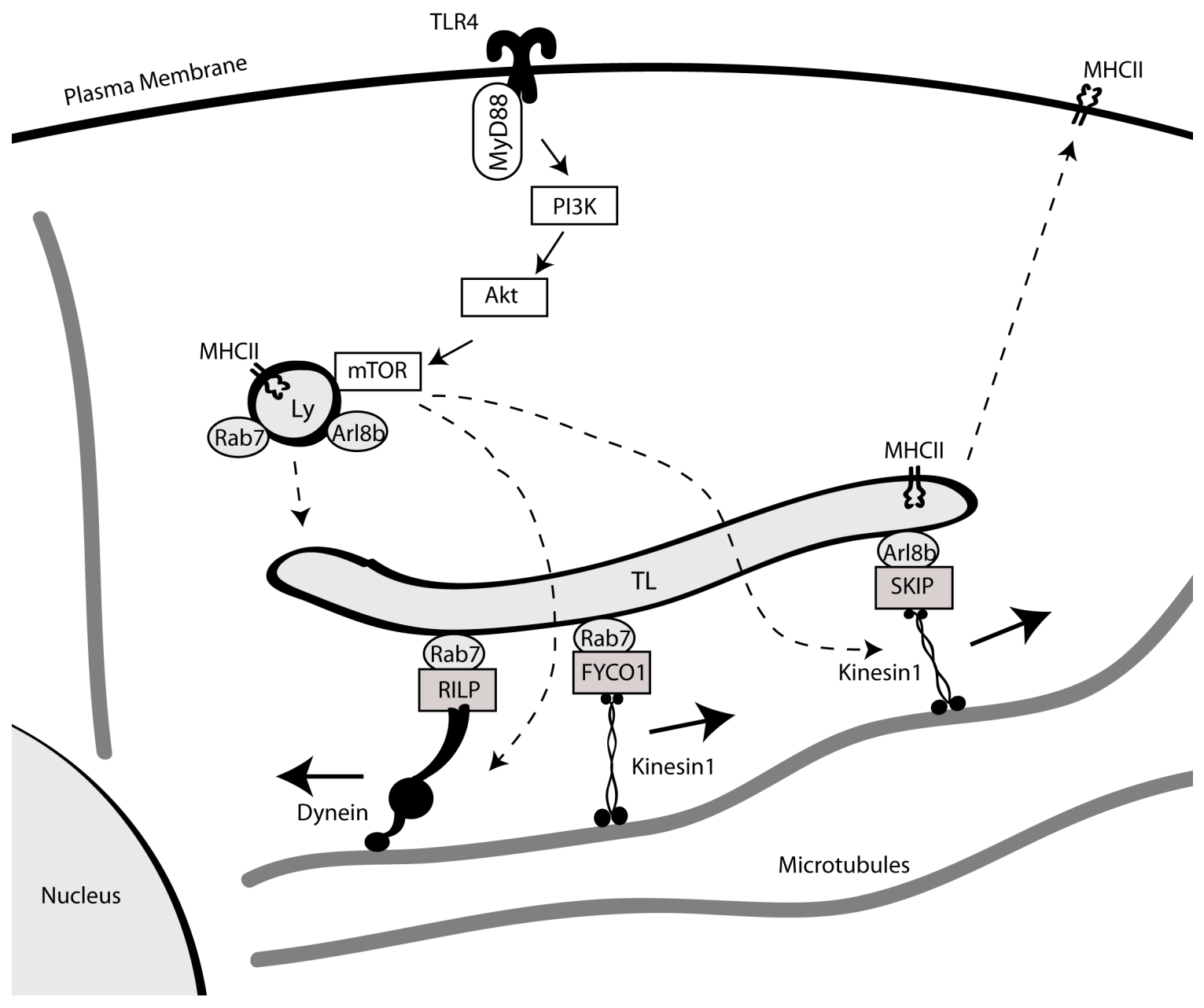


Chapter 4

Discussion 
4.1: Rab7 and Arl8 GTPases are Necessary for Lysosome Tubulation in Macrophages

Lysosomes and the related MIIC normally appear as small punctate structures in resting macrophages and DCs respectively. However, upon exposure of these cells to activating agents such as LPS, fungal antigens and phorbol esters, lysosomes and MIICs undergo a dramatic metamorphosis that is visually striking, where they transform into long tubular lysosomes and tubular MIICs (TLs and tMIIC) (Boes et al., 2003; Mrakovic, Kay, Furuya, Brumell, \& Botelho, 2012; Swanson, Yirines, \& Silverstein, 1985; Vyas et al., 2007). It has been proposed that the tMIIC functions to deliver antigen-bound MHCII complexes to immunological synapses (Bertho et al., 2003; Boes et al., 2002, 2003). TLs in macrophages appear to retain fluid-phase content, as resting macrophages lose approximatey $50 \%$ of their pinocytosed fluid compared to activated macrophages (Swanson, Yirinec, \& Silverstein, 1985; Swanson, Burke, \& Silverstein, 1987) and other studies implicate TLs in phagosome maturation and acidification and NOD 2 signaling (Harrison, Bucci, Vieira, Schroer, \& Grinstein, 2003; Nakamura et al., 2014; Sun-Wada, Tabata, Kawamura, Aoyama, \& Wada, 2009). Surprisingly, other than a requirement for microtubules and kinesin, very little was known about how TLs and tMIICs form (Boes et al., 2003; Swanson, Bushnell, \& Silverstein, 1987; Vyas et al., 2007). Using the RAW 264.7 macrophage cell line I demonstrate that LPS induces the transformation of lysosomes into tubular lysosomes and exploit this model system for the study of TL biogenesis.

The data presented in section 3.1 of this thesis suggests that TL biogenesis occurs through the coordinated action of the microtubule motor proteins dynein and kinesin. Thus we speculate that tubulation occurs in part by 'stretching', as lysosomes are pulled in opposite directions along microtubules by these motors. Given that TLs can reach excessive lengths, sometimes 15-20 $\mu \mathrm{m}$ long, we hypothesize TLs are generated from enlarged lysosomes that have experienced increased membrane fusion or decreased membrane fission. Thus, we envision that these enlarged lysosomes are stretched along microtubule scaffolds by dynein and kinesin to generate TLs. In support of this reasoning, large spherical lysosomes are frequently observed when microtubules, or the link between lysosomes and 
microtubules is perturbed (Figure 3.1.2), consistent with loss of a scaffold for tubulation. Alternatively, it is possible that LPS increases the number of lysosomes, thus providing more membrane for tubulation.

This work provides evidence that the lysosomal small GTPases Rab7 and Arl8b are essential for TL biogenesis. Given that FYC01, RILP and SKIP appear to play roles in TL formation, it is likely that Rab7 and Arl8b are modulating dynein and kinesin activity during TL biogenesis. Rab7 recruits RILP and FYCO1 to lysosomes, which are adaptors of dynein and kinesin respectively. Arl8b recruits SKIP and this effector directly binds kinesin to modulate kinesin-dependent trafficking of lysosomes and lysosome-related organelles (Bagshaw, Callahan, \& Mahuran, 2006; Kaniuk et al., 2011; Rosa-Ferreira \& Munro, 2011). Beyond a role in governing microtubule-associated motors, Rab7 and Arl8b play other important roles in lysosomal regulation. Specifically, both GTPases have been shown to associate with the HOPS (homotypic fusion and protein sorting) complex on lysosomes (Khatter et al., 2015; Wurmser, Sato, \& Emr, 2000). This complex is necessary for lysosome membrane fusion (Garg et al., 2011). Thus, it remains unclear whether these GTPases influence TL formation by controlling membrane trafficking.

In addition to their striking change in morphology, overall lysosomal motility is significantly increased upon formation of TLs. In section 3.1.7, I provide evidence that TLs are more often in a motile phase, compared to punctate lysosomes. This is obvious when TL mean velocities are computed, which averages the speed over periods of motile and static phases. Importantly, all lysosomes, regardless of their morphology, migrate at similar speeds, thus TLs do not experience faster overall speeds. Instead, the data indicates that punctate lysosomes undergo longer periods of latency and static behaviour, while TLs are more often in a motile phase. Previously, Falcon-Perez et al. reported that lysosomes labeled with fluorescent dextrans, as was done here, exhibited mainly short-range movement relative to LAMP1-GFP-labelled organelles, which displayed a mixture of both long-range and short-range movement (Falcón-Pérez, Nazarian, Sabatti, \& Dell'Angelica, 2005). This 'short-range' and 'long-range' movement may be indicative of the 'static' and 
'motile' phases described here. Thus, in agreement with previous studies, it appears that punctate lysosomes are more often in the 'short-range' or 'static' phase, while we observe TLs to be in the 'long-range' or 'motile' phase.

Finally, these data imply that regardless of LPS treatment, TLs always experienced a similarly enhanced mean lysosome velocity compared to punctate lysosomes. Because resting macrophages experience some basal lysosome tubulation and LPS-activated macrophages still retained some punctate lysosomes, we used these incomplete phenotypes to our advantage and measured mean lysosome velocities of punctate lysosomes and tubular lysosomes with and without LPS treatment (Figure 3.7E). This analysis was able to discern that LPS is not acting to directly hyperactivate lysosomal movement. Rather, LPS initiates a signaling cascade that increases tubulation and tubulation itself is associated with hypermotility. While the exact mechanism behind the enhanced motility of TLs is unknown, this data implies several possibilities: 1) TLs have greater levels of active motor proteins associated with them and/or 2) these motor proteins are more processive and/or 3) their activity is better coordinated. Alas, bidirectional movement of organelles is a poorly understood phenomenon (Kardon \& Vale, 2009). Perhaps tubular lysosomes will prove a good model system for the study of these processes. 
4.2: mTOR Regulates Lysosome Tubulation and Antigen Presentation in Macrophages and Dendritic Cells

Continuing on the investigation of tubular lysosome biogenesis in macrophages and DCs, I propose a role for the lysosomal protein kinase and major cell stress sensor, mTOR, in regulating lysosome tubulation by integrating signals downstream of TLR4 to the cellular machinery that drives lysosome tubulation. In addition to the proposed function of TLs in delivering MHCII-antigen complexes to the surface of antigen presenting cells (Boes et al., 2002, 2003) and remodeling phagosomes (Mantegazza et al., 2014; Stephen et al., 2007), I have shown in chapter 3, section 3.1 that TLs are highly motile compared to punctate lysosomes, which may enhance trafficking rates to lysosomes (Mrakovic, Kay, Furuya, Brumell, \& Botelho, 2012). Interestingly, little is known about how LPS-mediated signaling interfaces with molecular machinery that governs lysosomal morphology and identity.

Here I discuss my interpretation of the data presented in section 3.2 from which I propose a model where the TLR4-MyD88-PI3K-Akt-mTOR signaling pathway helps trigger lysosome tubulation in response to LPS in macrophages and DCs. This is supported by evidence that pharmacological inhibition of MyD88 and PI3K, in particular the Class I PI3Ks, Akt and mTOR all lead to a strong impediment in TL formation in macrophages and DCs. Conversely, inhibition of TBK1 or IRAK1/4 had no impact on lysosome tubulation. This finding is consistent with LPSdependent activation of Akt and mTOR, independently of IRAK1/4 or TBK1 (Figures 3.10 and 3.11). Of note, the data presented here is in agreement with previous work showing that MyD88, an adapter protein that can link TLR4 to PI3K-Akt, is required for lysosome tubulation (Bauerfeld et al., 2012; Boes et al., 2003; Laird et al., 2009).

It remains possible that TL biogenesis is regulated by multiple signaling pathways. Vyas and colleagues have previously shown that a fungal pathogen, Cryptococcus neoformans, which engages both TLR2 and TLR4, can induce extensive tubulation of endolysosomes, in a MyD88-independent manner in DCs (Vyas et al., 2007), though it is possible that this still proceeds through the PI3K-Akt-mTOR module. It may be that multiple signals converge on mTOR to induce tubulation, 
such as those derived from phorbol esters and T-cell-MHCII engagement. Indeed, others have shown an upregulation of Akt and mTOR activity in multiple cell types in response to phorbol esters (Aeder, Martin, Soh, \& Hussaini, 2004; Hartmann et al., 2013; Ni et al., 2015).

Having shown that the PI3K-Akt-mTOR axis controls LPS-induced TL biogenesis, we next asked how mTOR governs this event. We thus examined the factors known to be required for TL biogenesis and whether or not inhibition of mTOR causes changes to these factors. We began with the very template required for TL formation, microtubules. There appeared to be no observable difference in the gross morphology of microtubules or the basal motility of lysosomes (Appendix Figure A-3, A and B) in cells inhibited for mTOR. These findings suggested that mTOR does not control overall microtubule architecture or the basal motor activity associated with lysosomes in macrophages. However, mTOR inhibition did affect stimulus-dependent changes in lysosome positioning and motility. As described in chapter 3 , sub-section 3.2.7, lysosomes can be forced to the cell periphery upon cytosol acidification, while subsequent neutralization allows for a recovery period in which lysosomes return to the center of cells (Heuser, 1989). Interestingly, mTOR inhibition blocked acid-induced anterograde lysosomal transport but had no observable impact on retrograde transport as recovery of control and mTORinhibited cells showed a comparable lysosomal distribution. This suggests that mTOR may respond to certain stimuli to switch the equilibrium between anterograde and retrograde lysosomal transport. I further interpret this to mean that mTOR can stimulate kinesin and/or repress dynein, either directly or indirectly, to assist lysosomal anterograde transport. Unfortunately, the morphological changes lysosomes undergo upon LPS stimulation confounded this analysis and the LPS condition could not be analyzed. Nonetheless, I speculate that mTOR helps to coordinate kinesin and/or dynein to induce lysosome tubulation during LPS stimulation.

The mechanism by which mTOR may control motor activity remains unknown. It is possible that mTOR directly phosphorylates motor subunits to control their activity, or indirectly regulates their activity through modulation of 
other factors that control motor activation or anchoring to lysosomes. To test this, Victoria Hipolito in our lab transfected RAW cells with RFP-Rab7, RILPc33-GFP (a probe for GTP-Rab7) or Arl8b-GFP and assessed overall lysosomal localization of the GTPases under control, LPS or torin1+LPS conditions. While Rab7 and RILPc33 localization to lysosomal membranes remained unchanged after LPS stimulation, Victoria found that LPS increased the association of Arl8b with lysosomal membranes compared to control resting cells (Figure 4.1, Figures section at the end of this chapter). Interestingly, inhibition of mTOR abolished this LPS-induced membrane-localization, but not below control levels (Figure 4.1). These data suggest that mTOR may control stimulus-dependent activation of lysosomal transport machinery to alter lysosome morphology, and is in line with results from chapter 3, sub-section 3.2.7. A more rigorous biochemical approach to determine the actual activation state (GTP- vs GDP-bound) of the GTPases under these conditions is required, and is part of ongoing work from myself and other members of the Botelho lab.

Indeed, there exist several connections between microtubule motors and regulatory machineries of mTOR. Recently, Pu et al have identified BORC (BLOCone-related complex), a novel lysosomal complex that recruits Arl8b to lysosomes (Pu et al., 2015). Interestingly, the complex appears to be required for anterograde lysosomal motility and tubulation. Among the components found to interact with BORC, the authors list Ragulator, a lysosomal complex responsible for the activation of mTORC1 (Pu et al., 2015). Since this study focused on characterizing the interactions between Arl8b and BORC, further research into the mechanism and requirements of BORC in mTOR activation would be required. In addition, Arl8b and Ragulator subunits have been shown to be necessary for the proper delivery of focal adhesion components to the plasma membrane during cell adhesion, further emphasizing a potential role of mTOR regulatory machinery in coordinating anterograde trafficking (Schiefermeier et al., 2014). Finally, mTOR complexes with and phosphorylates CLIP-170, a plus-end microtubule tracking protein, which controls the development of dendrites and arborization of neurons in a PI3Kdependent manner (Swiech et al., 2011). 
All of the aforementioned studies, and supporting data from Victoria Hipolito (Figure 4.1) implicate mTOR in microtubule-dependent transport, particularly, anterograde transport. These findings are consistent with our observations that mTOR is necessary for cell surface accumulation of MHCII in APCs and correlates with a role for mTOR in TL biogenesis. This in turn supports a proposed function of TLs in delivering antigen-MHCII complexes to the plasma membrane at immune synapses (Boes et al., 2002). The small GTPase Arl8b has already been shown to be required for MHCII delivery to the plasma membrane (Michelet et al., 2015), thus we speculate that LPS-induced activation of mTOR regulates Arl8b, either through BORC, or an unknown guanyl exchange factor, to coordinate kinesin I activity on lysosomes and drive tubulation and exocytosis.

Given the association of mTOR with the lysosomal membrane via mTORC1, it is a suitable candidate to govern lysosomal properties. One of the best-characterized functions of lysosomal mTOR is its ability to integrate signals derived from amino acid levels in cells (Bar-Peled, Schweitzer, Zoncu, \& Sabatini, 2012; Zoncu, Bar-Peled, et al., 2011). Clearly, mTOR is activated by other signals, including those from the plasma membrane such as growth factors and LPS, and thus may act on lysosomes to adapt their function and properties to these diverse stimuli. It is possible therefore that mTOR controls lysosome tubulation by mechanisms other than microtubule-motor activity, such as through control of membrane fission (Krajcovic, Krishna, Akkari, Joyce, \& Overholtzer, 2013).

Finally, mTOR is central to growth control and clearly senses nutrients via the lysosome as part of mTORC1. It is widely known that mTOR and its regulation/deregulation is linked to multiple cancers, as a search in the PubMed database for "mTOR and cancer" yields nearly 10, 000 studies. Some of these implicate mTORC1 in cancer via its promotion of protein translation and suppression of autophagy, as well as ageing, where dietary restriction, which extends the lifespan of mice, acts mainly via suppression of mTORC1 (reviewed in Zoncu, Sabatini, \& Efeyan, 2011). Indeed lysosomes and alterations in their functions have also been linked to ageing (Cuervo \& Dice, 2000). Interestingly, mTORC1 can also directly influence cancer cell growth and survival, independent of 
translation and autophagy regulation. A study from earlier this year in Oncogene identified a direct interaction between mTORC1 and ER $\alpha$ (Estrogen Receptor $\alpha$ ), a transcription factor present in almost two thirds of breast cancers that promotes growth and survival of cancer cells in an estrogen-dependent manner (Alayev et al., 2015). In this study, the authors describe an interaction between Raptor (mTORC1 subunit) and $E R \alpha$, which results in direct phosphorylation and activation of $E R \alpha$ by mTOR leading to expression of growth and survival genes (Alayev et al., 2015).

Given the association of mTORC1 and lysosomes and the work of this thesis implicating for the first time regulation of lysosomal morphology by mTOR in innate immune cells, it remains to be tested whether other physiological or pathological conditions lead to lysosome tubulation in other cell types. I believe it would be of great interest to investigate if/how lysosomal morphology is altered in disease states like cancer and ageing and whether or not lysosome morphology contributes to disease progression. For example, lysosomes of the highly transformed human epithelial cell line, HeLa, appear highly tubular under resting conditions (unpublished data, Danielle Johnson \& Sergio Grinstein). Indeed, it is possible that lysosome tubulation is intimately linked with a number of diseases in which mTOR is deregulated, including cancer, but has remained overlooked. For example, lysosomal tubules are sensitive to common fixation methods like those relying on paraformaldehyde or methanol, and completely fragment under these conditions. In addition, unless specifically labeled with a bona fide lysosomal marker, the tubules could go unnoticed. 
4.3 Preliminary Data and Discussion on the Physiological Properties of Tubular Lysosomes

\subsubsection{Trafficking of Endocytic Cargo to Tubular Lysosomes}

Given the striking phenotype of tubular lysosomes, we questioned if the morphological change translated to a physiological change to the identity of the organelle for the purposes of acutely fine-tuning lysosomal properties when needed. For example, the motility of tubular lysosomes is greater than punctate lysosomes (Figure 3.7). That is, tubular lysosomes are on the move more often than punctate lysosomes. Could there be biological implications for this? We speculated that tubular lysosomes might acquire incoming cargo faster than punctate lysosomes, by virtue of their increased motility.

I sought to test the acquisition of incoming endocytic cargo by tubular lysosomes and compare these rates to those of punctate lysosomes. RAW cell lysosomes were pre-labeled with Alexa555-dextran (red) and lysosome tubulation was stimulated with LPS. Then, a second Alexa488-dextran (green) was pulsed for 5 minutes to allow for internalization of this second dextran into endocytic vesicles, followed by 0,5 or 20 minute chase times at which points the cells were observed under the fluorescence microscope. The resulting micrographs were analyzed by manually drawing regions of interest (ROIs) around tubular lysosomes and punctate lysosomes within the red channel (lysosomes). We then computed whether each ROI at each time point was positive for any green signal above background (schematic in Figure 4.2A). Using this approach, the data suggest that tubular lysosomes acquire fluid-phase endocytic cargo at the same rates as punctate lysosomes as there was no significant difference in the percentage of lysosomes containing green dextran at the chase times indicated, between the two lysosome populations (Figure 4.2B). Thus, the data suggest that the increased motility of tubular lysosomes is likely not for the purposes of acquiring incoming endocytic cargo at enhanced rates, though this remains to be tested for receptor-mediated 
cargo. Interestingly, with both types of lysosomes, a striking $40 \%$ of lysosomes already contained the second dextran at chase time zero. This indicates that RAW macrophages can deliver endocytosed fluid-phase cargo to lysosomes within only 5 minutes.

\subsubsection{Lysosome Tubulation and Lumenal Volume}

Under the fluorescence microscope, tubular lysosomes appear to traverse more of the cell cytosol than punctate lysosomes do. For example, when assessing the appearance of lysosomes in unstimulated macrophages and dendritic cells, fluorescent fluid-phase markers of the lumen feature defined "spherical" structures throughout the cell. However, upon activation with LPS and conversion of these compartments into long tubular networks, lysosomal lumen appears to spread throughout the cell within these defined tubules. It appears as though LPSstimulated cells harbor an expanded lysosomal lumen and that lumen appears heavily interconnected. These observations prompted us to consider that tubulation, as a biophysical phenomenon, might be a mechanism by which these cells expand and perhaps homogenize their lysosomal lumen.

To preliminarily test the expansion of volume, we used primary mouse BMDCs, as LPS activation induces a robust lysosome tubulation phenotype which is typically much stronger than in RAW cells. Lysosomes of immature DCs were labeled with Alexa555-dextran by pulse-chase and images of cells were acquired by confocal fluorescence microscopy. The same was done for DCs that were treated with LPS to induce lysosome tubulation, after lysosomes were labeled. Importantly, z-stacks were acquired for all the cells. That is, individual confocal slices in the zaxis were acquired in order to reconstruct a 3-dimensional image of each cell. This allowed us to measure the number of voxels (voxel count) containing the fluorescent signal, where a voxel is a unit of value within a 3D dataset, analogous to a pixel in a 2D dataset. A 3D rendering of immature and LPS-activated DCs is presented in Figure 4.3A. Interestingly, and consistent with the idea that lysosome 
tubulation increases the total lysosomal volume, the average voxel count was significantly higher in highly-tubulating cells compared to immature DCs (Figure 4.3B).

Although preliminary, these data suggest that lysosome tubulation expands the total lysosomal volume and by extension, membrane surface area of this compartment as well. This prompts the question, where is this extra source of membrane coming from? The likely explanations are increased membrane fusion, decreased membrane fission, or both. Indeed, several preliminary experiments seem to suggest that both mechanisms might be occurring simultaneously. The evidence for increased membrane fusion comes from the observation that blocking incoming endosomal membrane trafficking by expressing a dominant negative Rab5 GTPase (Rab5 $\left.{ }^{S 34 N}\right)$, an essential component of early endosomal trafficking, significantly suppresses lysosome tubulation (Figure 4.4A). This result suggests that membrane trafficking through the early endosomal compartments is required for lysosome tubulation, perhaps because it is a major pathway contributing membrane to lysosomes during the process of forming tubules. In addition, treatment of cells with the microtubule-depolymerizing agent nocodazole, which causes the collapse of TLs due to the loss of a microtubule scaffold, results in apparently enlarged lysosomes throughout the cytosol (Figure 4.4B). This preliminary experiment, which is not without caveats like the limit of resolution in light microscopy, appears to suggest that LPS signaling, which led to the formation of lysosomal tubules, increased membrane fusion to this compartment, resulting in enlarged organelles after microtubule loss.

Finally, preliminary data from experiments in which the membranescissioning GTPase dynamin is blocked with the pharmacological inhibitor dyngo, appears to induce tubulation of lysosomes alone (Figure 4.4C). This was an interesting result as it suggests that dynamin may function in lysosomal fission and that tubulation can occur through simply down-regulating this GTPase. Thus, it is possible that, in addition to the signals invoked by LPS to cause lysosome tubulation, dynamin down-regulation might be one of them. Collectively, these data provide 
evidence for both increased membrane fusion and decreased membrane fission as mechanisms of expanding lysosomal volume during TL biogenesis.

Why do DCs expand their lysosomal compartments?

A plausible physiological function of increasing total lysosomal volume via what appear as inter-connected networks of tubules might be to homogenize cargo. Homogenization of lumenal content might serve to allow for enhanced sampling of antigen in professional APCs like DCs. For example, due to high polymorphism in the MHCII peptide-binding groove, all MHCII molecules will slightly differ from one another in the antigens they can bind. In addition, MHCII molecules are sorted to lysosomes that, due to the variability in MHCII affinities for different antigens, could by extension generate slight heterogeneity among lysosomes, in terms of the types of antigen each lysosome's MHCII molecules are capable of binding. These factors, combined with trafficking of different antigens to individual lysosomes might statistically limit the possibilities of antigen-MHCII combinations. However, if all the slightly heterogeneous lysosomes were to fuse into an expanded, inter-connected tubular network, this would allow for free diffusion of antigens throughout the lumen and free lateral diffusion of MHCII molecules in the lysosomal membrane, thus increasing the possibility that all the various MHCII molecules are accessible to all the various antigens taken up by the cell.

Though it would be technically challenging to test an effect on antigen presentation, a first step would be to test the homogenization hypothesis. We envision using a photo-activatable probe that targets to lysosomes. Using this probe, we would photo-activate one half of a cell, which would fluorescently label half of all lysosomes. After this, we would monitor the spread of the fluorescent signal throughout the lysosomes of the cell, over time. This would be conducted with cells that are highly tubulating their lysosomes and compared with cells with mostly punctate lysosomes. We expect that highly-tubulated lysosomes will spread the fluorescent signal across the cell faster than punctate, non-connected lysosomes would. This work is currently ongoing and in preparation for these experiments we 
have acquired a photo-activatable GFP-LAMP1 plasmid construct to transfect into RAW cells. Alternatively, lysosomal homogenization could be quantified by prelabeling lysosomes of a cell with one fluorescent marker (ie. Alexa555-dextran), prelabeling lysosomes of another cell with a different fluorescent marker (ie. Alexa488dextran) and conducting a cell-cell fusion assay using the fusogen polyethylene glycol (PEG) (Yang and Shen, 2006). The colocalization of two markers would be assessed in untreated and LPS-treated cells. This experiment would serve to enhance our knowledge of whether LPS induces homogenization of lysosomal content via homotypic lysosomal fusion.

\subsection{Additional Contributions}

During the course of my graduate studies, I have mainly focused on my projects on the elucidation of tubular lysosome biogenesis in innate immune cells, as this thesis describes. This work has resulted in two first-author publications:

Mrakovic, A. , Kay, J.G., Furuya, W., Brumell, J.H., Botelho, R.j. (2012) Rab7 and Arl8 GTPases are necessary for lysosome tubulation in macrophages. Traffic 13:16671679.

Saric, A., Hipolito, V.E.B., Kay, J.G., Canton, J., Antonescu, C., Botelho, R.J. (2015) mTOR controls lysosome tubulation and antigen presentation in macrophages and dendritic cells. Molecular Biology of the Cell [Epub ahead of print].

However, unique opportunities for collaboration with peers have presented themselves over the years and I was happy to lend expertise to my collaborators toward the completion of several other projects. The following works are additional projects I have contributed to. I briefly describe the extent of each of my contributions.

${ }^{*}$ Note: Some publications contain my previous last name, Mrakovic. 
The following article was published in Journal of Biological Chemistry:

Alghamdi, T., Ho, C., Mrakovic, A., Taylor, D., Mao, D., Botelho, R.J. (2013) Vac14 protein multimerization is a prerequisite step for Fab1 protein complex assembly and function. Journal of Biological Chemistry 288(13):9363-72.

This project involved determining the multimeric state of the Vac14 adaptor protein, which is required for the proper functioning of the Fab1 complex. This complex regulates synthesis of $\mathrm{PI}(3,5) \mathrm{P}_{2}$ on vacuole membranes and therefore is a major contributor to establishing organelle identity in yeast. Most of my contributions to this work were the result of experiments I conducted during my undergraduate thesis, specifically the generation of truncated Vac14 mutant yeast expression vectors ultimately used for expression and immunoprecipitation work. However, as a PhD student I contributed to the completion of this work by providing manuscript inputs and editing.

The following article was published in Traffic:

Dayam, R. M., Saric, A., Shilliday, R. E., Botelho, R. J. (2015) The phosphoinositidegated lysosomal $\mathrm{Ca}{ }^{2+}$ channel, TRPML1, is required for phagosome maturation. Traffic 16(9):1010-1026

This project was on the elucidation of the role of a phagosomal $\mathrm{Ca}^{2+}$ channel, TRPML1 (transient receptor potential cation channel, mucolipin subfamily, member 1), in phagosome maturation. Indeed, we found that this channel is necessary for the fusion of phagosomes with docked lysosomes, likely due to its ability to release phagosomal $\mathrm{Ca}^{2+}$ stores, which we reason is the fusogenic signal that completes phagosome maturation. My contribution to this work has been to show that a delay in phagosome maturation in TRPML1-deficient cells is specifically due to loss of the channel and not an effect of altering phosphoinositide dynamics on phagosomes. 
Thus I conducted the appropriate experiments, quantified data and generated "Figure 3" in the article. A snapshot of this figure is included here:

"The phosphoinositide-gated lysosomal Ca 2+ channel, TRPML1, is required for phagosome maturation"

Figure 3
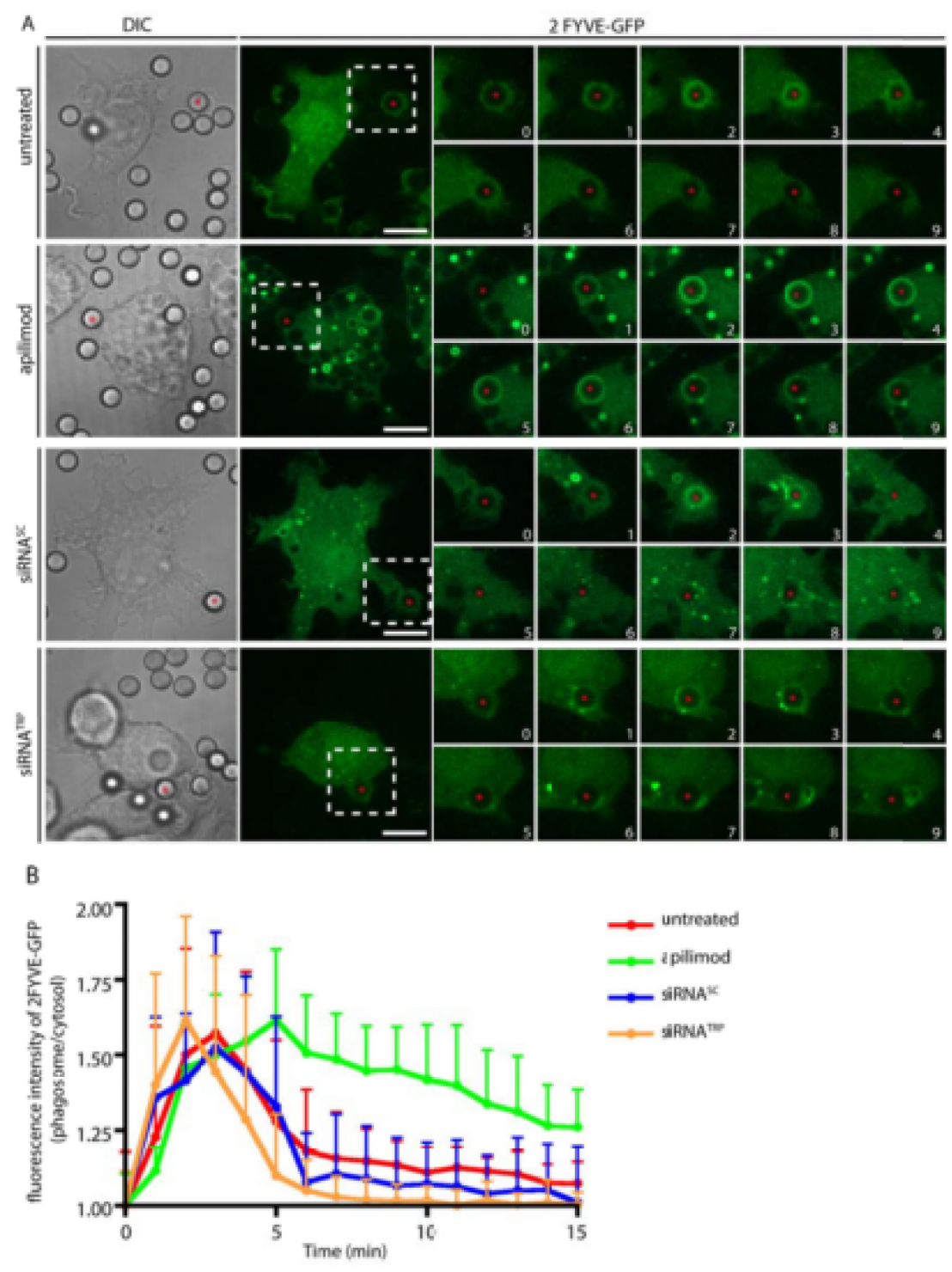
The following manuscript is under review in Cell Reports.

Wong, H., Jaumouille, V., Freeman, S., Doodnauth, S., Schlam, D., Canton, J., Mukovozov, I., Saric, A., Grinstein, S., Robinson, L. (2015) CX3CL1 enhances CD36 responsiveness towards oxidized low-density lipoproteins and accelerates foam cell formation. Cell Reports. Manuscript number: D-15-01971

My contribution to this work was the bone marrow harvesting and differentiation of mouse macrophages used in the study, from wild-type and CD36\% mice. In addition, I contributed to discussion of the experiments and scientific merit of the work with the primary author. 
Figures for Chapter 4 
Figure 4.1: LPS Increases the Levels of Membrane-Associated Arl8b in an mTORDependent Manner. (A) Quantification of fluorescence micrographs of membraneassociated RFP-Rab7 (black bars) or RILPc33 (grey bars). Quantification is a ratio of fluorescence intensity in membrane (defined by overlapping with fluorescent lysosomal dextran) to the fluorescence intensity in the cytosol, normalized to cells unexprosed to LPS. (B) Fluorescence micrographs of RAW cells expressing Arlb-GPF and lysosomes labeled with Alexa555-dextran. Scale bar $=10 \mu \mathrm{m}$. (C) Quantification of membrane-associated Arl8b0GFP signal as in A. Data are mean \pm SEM. Data was statistically analyzed using a one-way ANOVA, followed by Tukey's post-hoc test. An asterisk $\left(^{*}\right)$ indicates significant difference between DMSO+LPS and DMSO conditions $(p<0.001)$. Note: data was collected and analyzed by Victoria Hipolito. 
Figure 4.1

A

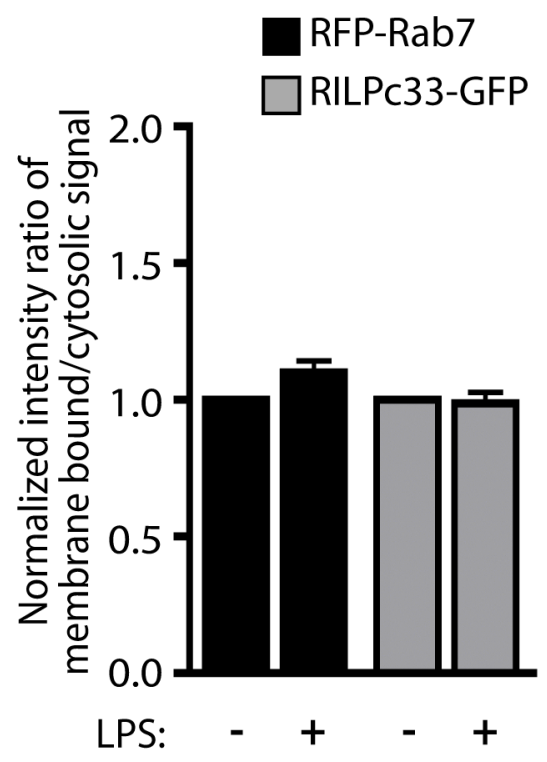

C

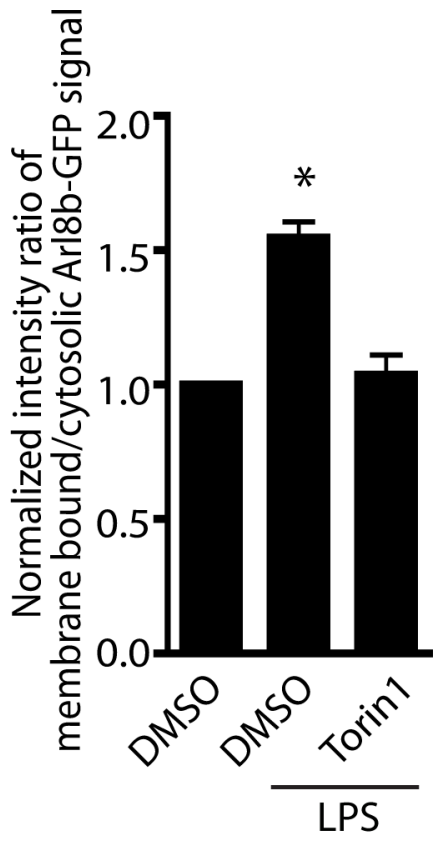

B
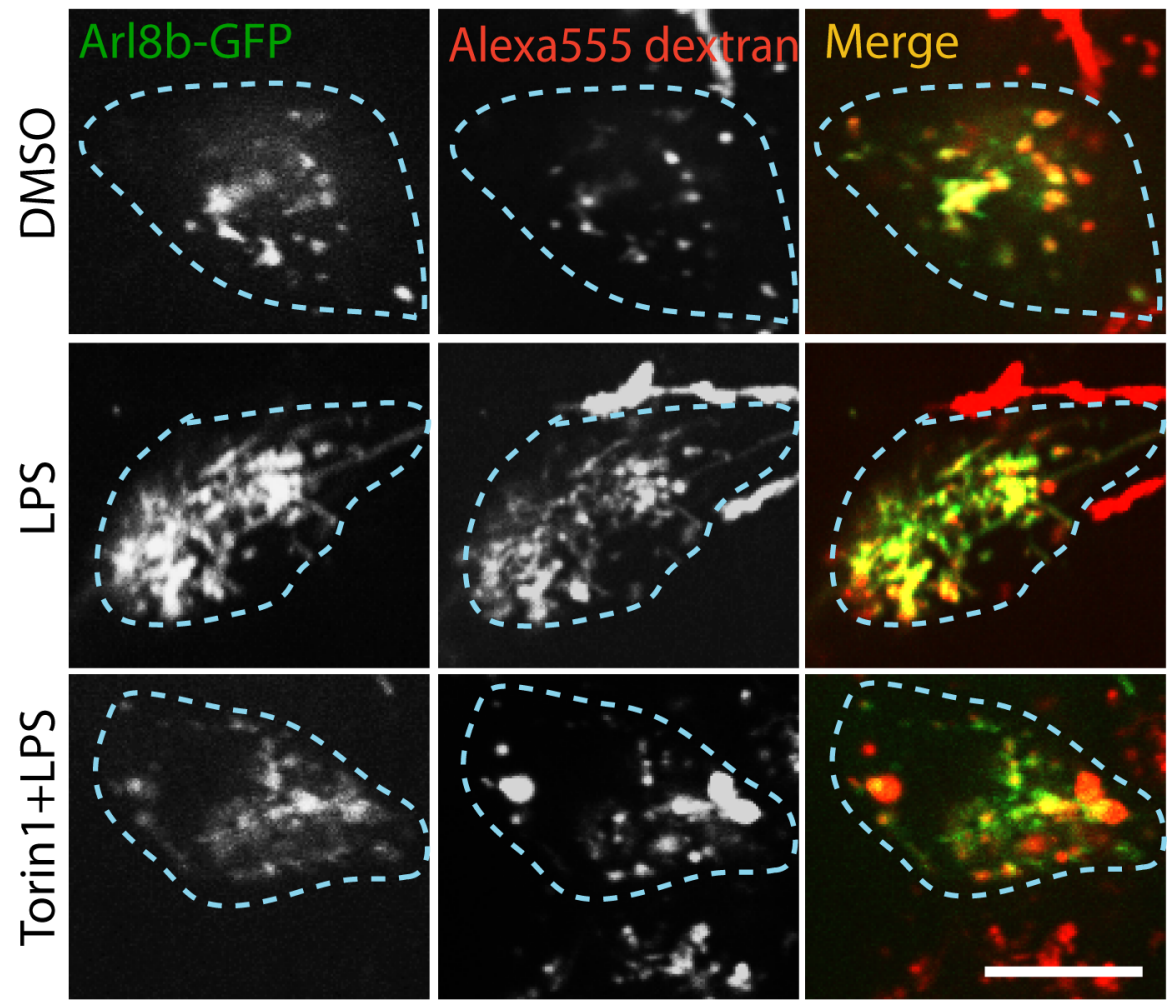
Figure 4.2: Endocytic Fluid-Phase Cargo Traffics to Tubular Lysosomes at the Same Rate as to Punctate Lysosomes. (A) Sample of method of measuring cargo trafficking to punctate and tubular lysosomes. RAW cell lysosomes were prelabeled with Alexa-555 dextran ( $0.5 \mathrm{~h}$ pulse, $1 \mathrm{~h}$ chase) followed by LPS stimulation to induce lysosome tubulation. Alexa-488 dextran was pulsed briefly ( $5 \mathrm{~min}$ ) to allow for uptake of this second dextran, followed by various chase times: $0 \mathrm{~min}, 5$ min or 20 min. Sample micrograph shows 20 min chase with second dextran. Individual tubular or punctate lysosomes were identified and outlined in the red channel (blue dashed lines). Volocity program was used to compute whether or not the outlined lysosomes contained green signal (second dextran pulse) at the chase times indicated. Lysosomes were positive for green if signal was above a threshold (background). (B) Graph of quantification using approach in $A$, among three individual experiments. Data are presented as the percentage of lysosomes containing second dextran at the chase times indicated. Data was analyzed by paired Student's t-test and was not significantly different between TLs and punctate lysosomes at any time point tested. 
Figure 4.2

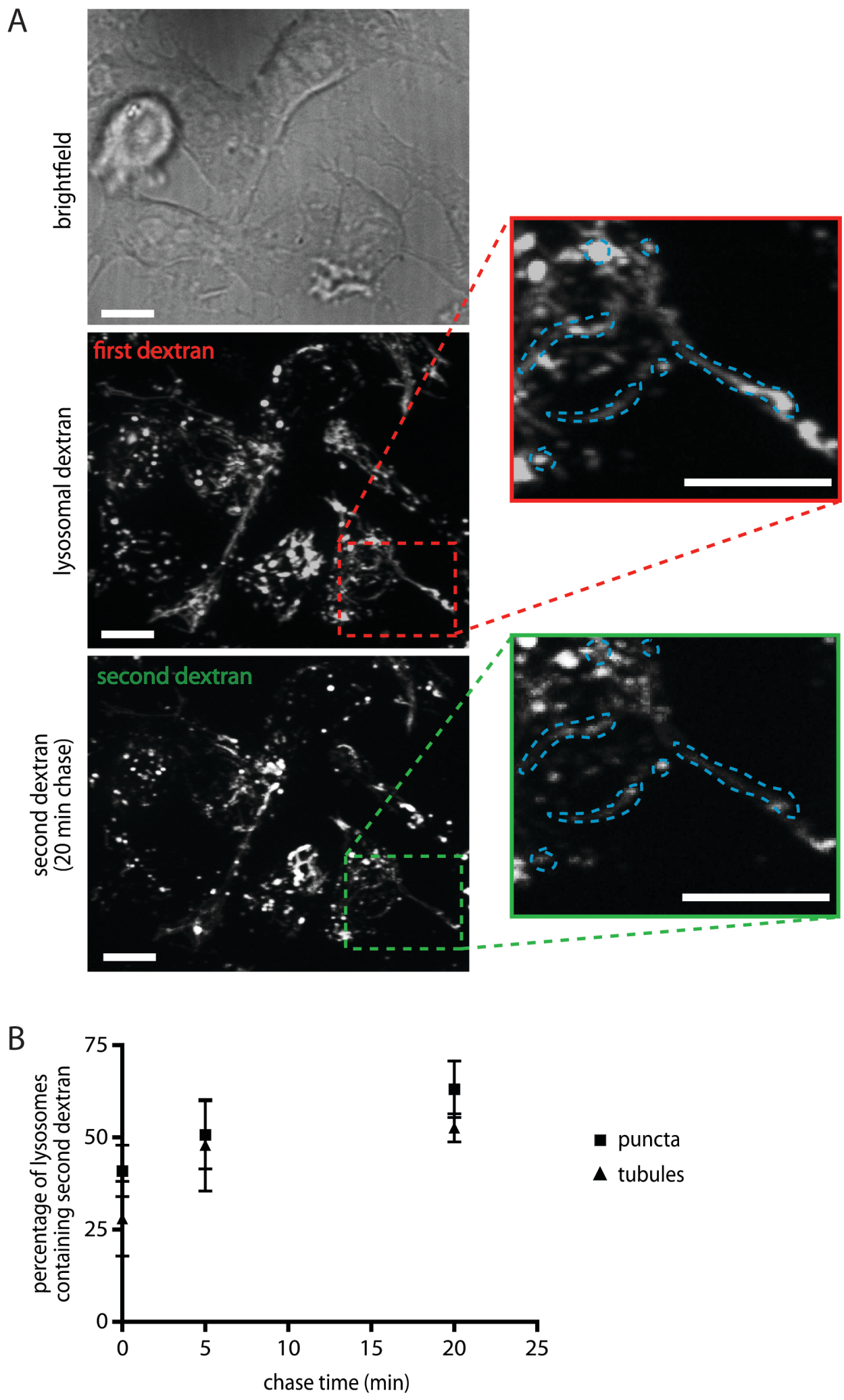


Figure 4.3: Total Lysosomal Volume Increases Upon Tubulation. (A) 3D rendering of unstimulated (left) and LPS-stimulated (right) dendritic cells in which lysosomes were pre-labeled with Alexa-555 dextran. Arrows point out lysosomal tubules. 3D images are a rendering of multiple confocal images spanning the z-axis and allow for easier visualization of lysosomal volume. (B) Lysosomal volume was computed as a total number of voxels that were positive for Alexa-555 dextran signal above a threshold value. Data is based on 70-75 cells analyzed per condition across 3 individual experiments. LPS condition is significantly greater than control with $\mathrm{p}=0.0012$ determined by Student's t-test. 
Figure 4.3
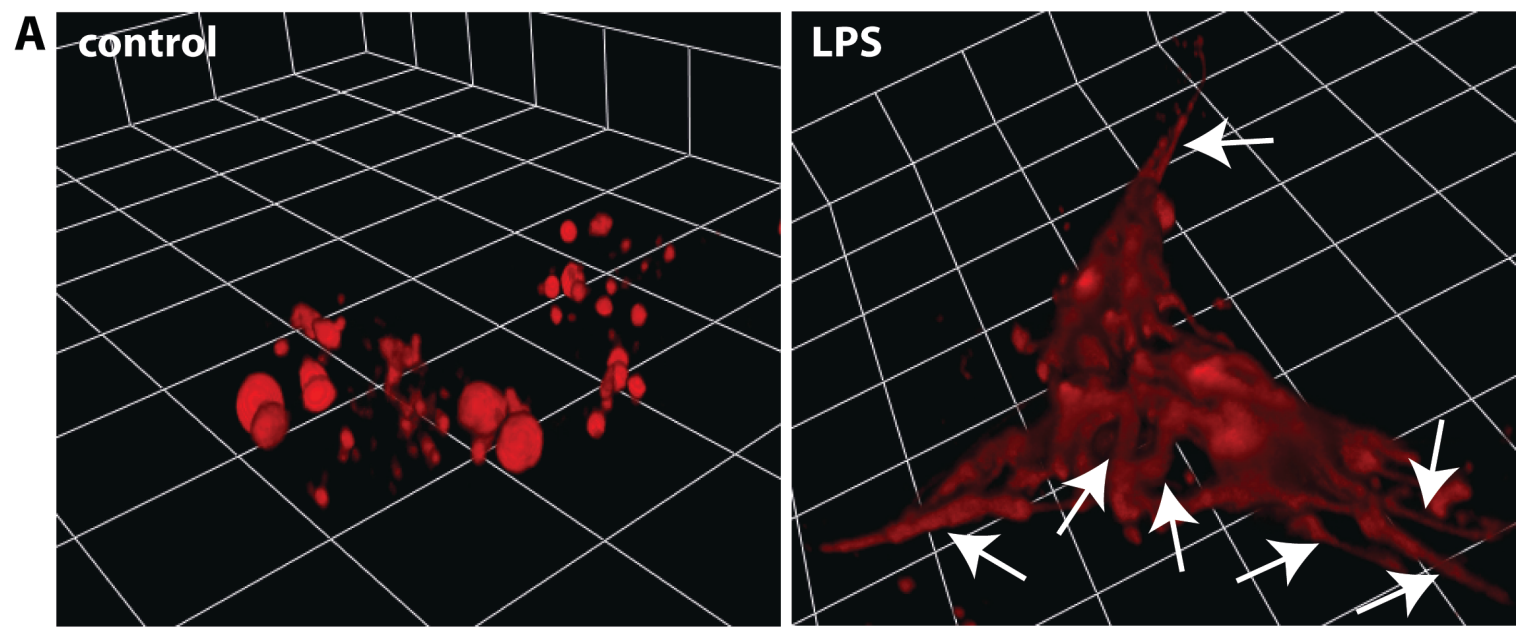

1 unit $=8.7 \mu \mathrm{m}$

B

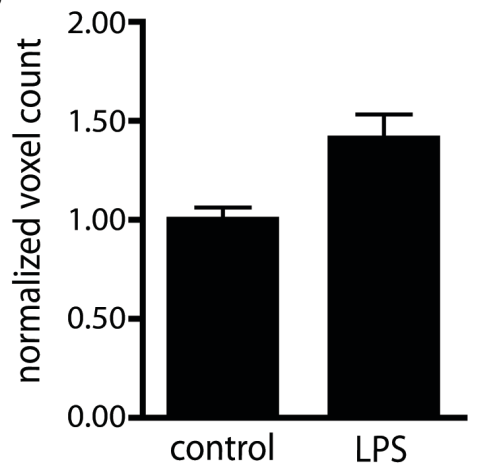


Figure 4.4 Evidence for Membrane Fusion and Fission in Regulating Tubular Lysosome Biogenesis. (A) Lysosomes of non-transfected, resting RAW cells and RAW cells transfected with a plasmid construct expressing dominant negative Rab5 (Rab5534N) were labeled with Alexa555-dextran and stimulated with LPS. The cells were imaged by fluorescence microscopy and the number of tubular lysosomes per cell was scored. (B) RAW cell lysosomes were labeled with Alexa555-dextran. Cells were either left untreated (-LPS, -Nocod), treated with LPS (+LPS, -Nocod), treated with nocodazole (-LPS, +Nocod) or both (+LPS, +Nocod) and imaged by fluorescence microscopy. Dashed cyan lines outline individual cells. Red arrowheads indicate lysosomal tubules, yellow arrowheads point out what appear as "enlarged" lysosomes. Scale bar $=10 \mu \mathrm{m}$. (C) RAW cells were either left untreated (control), or treated with the dynamin inhibitor dyngo for $2 \mathrm{~h}$, or treated with LPS for $2 \mathrm{~h}$, and lysosome tubulation was scored. Data are mean \pm SEM. In $A$, data is based on two individual experiments in which $\geq 25$ cells were quantified per condition, and Student's t-test was applied to determine significance, $p<0.0001$. In $B$, data is based on one experiment in which $\geq 20$ cells were quantified per condition. 
Figure 4.4
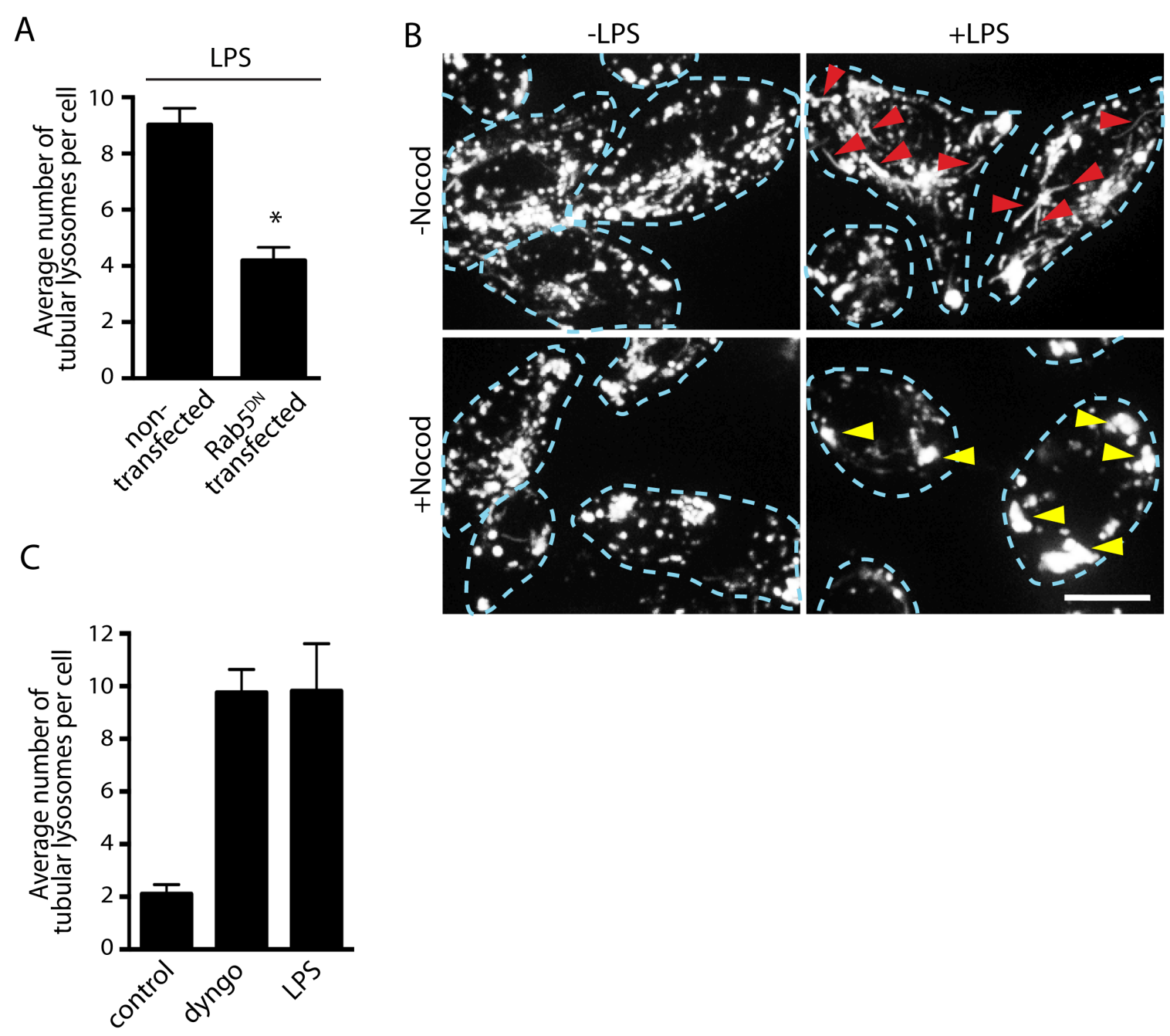
Conclusion 
In conclusion, this work presents evidence that LPS increases the tubular lysosome population in macrophages, which is a significantly more dynamic lysosomal population than "normal" punctate lysosomes. Moreover, this work is the first to identify key machinery of lysosome tubulation in macrophages. This machinery includes the lysosomal small GTPases Arl8b and Rab7, their effectors, SKIP, FYCO1 and RILP, which collectively recruit microtubule-based motor proteins dynein and kinesin to regulate this process. I envision that lysosome tubulation occurs through the concerted actions of these motors in "stretching" lysosomes along microtubule scaffolds and hypothesize that increased membrane fusion and/or decreased membrane fission are involved in providing the lysosomal membrane needed to convert small punctate lysosomes into long tubules.

Furthermore, this work identifies the signaling pathway downstream of the LPS receptor TLR4 that leads to lysosome tubulation in macrophages and dendritic cells. From the data presented in this thesis, I identify the TLR4-MyD88-PI3K-AktmTOR pathway as being the major signaling axis controlling lysosome tubulation in LPS-stimulated macrophages. Thus, I place mTOR at the major integration point of LPS signaling and the control of lysosomal morphology and trafficking. To my knowledge, this is the first description of a signaling pathway downstream of TLR4 that controls organelle morphology. I provide evidence that mTOR exerts its effects on lysosome tubulation by regulating anterograde lysosomal transport likely by modulating the activation state and membrane localization of the small GTPase Arl8b, the key GTPase responsible for anterograde lysosomal transport by kinesin I. This signaling axis was identified to be critical for LPS-stimulated antigen presentation by maturing dendritic cells, a process previously shown to require Arl8b and MIIC tubulation.

Finally, in exploring the physiological properties of tubular lysosomes, I provide evidence that tubulation may serve to expand the lysosomal lumen and homogenize lysosomal cargo, which could have implications in antigen processing, sampling and presentation in professional APCs. 
Overall, this thesis contributes to the fields of intracellular membrane trafficking, organelle identity and innate immune cell function by enhancing our existing knowledge of lysosomal morphology regulation in innate immune cells. 
Appendix 
Table A-1: Absolute Values of Lysosome Tubulation Number for Section 3.1. All values under 'replicates' column are average number of tubules per cell per replicate, where each row of values represents experiments conducted on different days. The 'mean \pm SEM' column shows the average number of tubules per cell calculated by pooling all cells together from all relevant replicates. Square brackets indicate type of control and type of experimental condition. Round brackets indicate the $\mathrm{p}$ value of experimental results, as analyzed by Student's t-test. Under silencing conditions, data may be present for an siRNA SMARTpool oligo mix or for individual oligos against the target specified under the 'condition' column. 
Table A-1

\begin{tabular}{|c|c|c|c|c|c|c|c|c|c|c|c|c|c|}
\hline $\begin{array}{l}\text { Figure \# } \\
\text { Reference }\end{array}$ & Condition & \multicolumn{6}{|c|}{ Replicates } & \multicolumn{6}{|c|}{$\begin{array}{c}\text { Mean } \pm \text { SEM } \\
\text { (Analyzed by Student's t-test) }\end{array}$} \\
\hline \multirow{9}{*}{$\begin{array}{l}\text { Figure } \\
3.2 \mathrm{D}\end{array}$} & \multirow{5}{*}{$\begin{array}{c}\mathrm{KIF5}^{\mathrm{DN}} \\
\text { overexpression }\end{array}$} & \multicolumn{3}{|c|}{$\begin{array}{c}\text { Control } \\
\text { [untransfected] }\end{array}$} & \multicolumn{3}{|c|}{$\begin{array}{l}\text { Experimental } \\
\text { [transfected] }\end{array}$} & \multicolumn{3}{|c|}{\begin{tabular}{c|} 
Control \\
[untransfected]
\end{tabular}} & \multicolumn{3}{|c|}{$\begin{array}{l}\text { Experimental } \\
\text { [transfected] }\end{array}$} \\
\hline & & \multicolumn{3}{|c|}{9.5} & \multicolumn{3}{|c|}{5.5} & \multirow{4}{*}{\multicolumn{3}{|c|}{$9.9 \pm 0.5$}} & \multirow{4}{*}{\multicolumn{3}{|c|}{$\begin{array}{c}6.0 \pm 0.6 \\
(p<0.0001)\end{array}$}} \\
\hline & & \multicolumn{3}{|c|}{13.2} & \multicolumn{3}{|c|}{10.0} & & & & & & \\
\hline & & \multicolumn{3}{|c|}{4.6} & \multicolumn{3}{|c|}{3.1} & & & & & & \\
\hline & & \multicolumn{3}{|c|}{8.7} & \multicolumn{3}{|c|}{4.2} & & & & & & \\
\hline & \multirow{4}{*}{$\begin{array}{c}\text { Dynamitin } \\
\text { overexpression }\end{array}$} & & 12.7 & & & 7.7 & & & & & & & \\
\hline & & & 14.6 & & & 10.4 & & & & & & $6.8 \pm 0.7$ & \\
\hline & & & 11.1 & & & 8.0 & & & $10.9 \pm 0.5$ & & & $(p<0.0001)$ & \\
\hline & & & 5.4 & & & 2.3 & & & & & & & \\
\hline & & [wild ty & $\begin{array}{l}\text { Control } \\
\text { Rab7-tra }\end{array}$ & ssfected] & & $\begin{array}{l}\text { Experiment } \\
b 7^{\top 22 N} \text {-transf }\end{array}$ & & [wild typ & $\begin{array}{l}\text { Control } \\
\text { e Rab7-trans }\end{array}$ & Isfected] & $\begin{array}{r}\mathrm{E} \\
{[\mathrm{Rab} 7}\end{array}$ & $\begin{array}{l}\text { Experimente } \\
7^{\mathrm{T} 22 \mathrm{~N}} \text {-transfe }\end{array}$ & $\begin{array}{l}\text { al } \\
\text { ected] }\end{array}$ \\
\hline Figure & $\begin{array}{c}\operatorname{Rab} r \\
\text { overexpression }\end{array}$ & & 12.8 & & & 3.9 & & & & & & & \\
\hline $3.3 \mathrm{D}$ & & & 8.2 & & & 3.3 & & & $8.7 \pm 0.6$ & & & & \\
\hline & & & 4.3 & & & 0.9 & & & & & & & \\
\hline & & & $\begin{array}{l}\text { Control } \\
\text { transfect }\end{array}$ & & & $\begin{array}{l}\text { Experiment } \\
\text { [transfected }\end{array}$ & & & $\begin{array}{c}\text { Control } \\
\text { ntransfected }\end{array}$ & & & $\begin{array}{l}\text { Experiment } \\
\text { [transfected }\end{array}$ & \\
\hline Figure & $\begin{array}{l}\text { RILP } \\
\text { overexpression }\end{array}$ & & 4.9 & & & 3.3 & & & & & & & \\
\hline $3.4 \mathrm{~B}$ & & & 3.0 & & & 1.1 & & & $4.2 \pm 0.4$ & & & $\begin{array}{c}2.4 \pm 0.4 \\
(D=0.0046\end{array}$ & \\
\hline & & & 7.5 & & & 3.3 & & & & & & & \\
\hline & & $\begin{array}{r}\mathbf{s} \\
\text { [scramb }\end{array}$ & $\begin{array}{l}\text { NA oligo } \\
\text { d control }\end{array}$ & $\begin{array}{l}11 \\
\text { vs. oligo] }\end{array}$ & [scramt & $\begin{array}{l}\text { siRNA oligo } \\
\text { bled control }\end{array}$ & S. oligo] & $\begin{array}{r}\mathrm{sil} \\
\text { [scrambl }\end{array}$ & $\begin{array}{l}\text { RNA oligo } 1 \\
\text { ed control v }\end{array}$ & IS. oligo] & $\begin{array}{r}\mathrm{si} \\
\text { [scrambl }\end{array}$ & $\begin{array}{l}\text { iRNA oligo } \\
\text { led control }\end{array}$ & $\begin{array}{l}12 \\
\text { Vs. oligo] }\end{array}$ \\
\hline Figure & & scramb & & oligo 11 & scramb & \begin{tabular}{l|l} 
bled & $\mathrm{c}$ \\
\end{tabular} & go 12 & scrambl & & oligo 11 & scrambl & & Oligo 12 \\
\hline $3.4 \mathrm{D}$ & silencing & 4.2 & & 1.7 & 2.7 & & 0.5 & & & $18+03$ & & & $12+02$ \\
\hline & & 3.5 & & 1.1 & 3.5 & & 3.3 & $4.6 \pm 0$. & & $1.0 \pm 0.0$ & $3.7 \pm 0$. & & $1.2 \pm 0.2$ \\
\hline & & 5.9 & 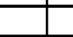 & 2.5 & 5.9 & \begin{tabular}{|l|l}
$y$ & \\
\end{tabular} & 1.1 & & & & & & \\
\hline & & & $\begin{array}{l}\text { Control } \\
\text { transfect }\end{array}$ & & & $\begin{array}{l}\text { Experiment } \\
\text { [transfectes }\end{array}$ & & & $\begin{array}{c}\text { Control } \\
\text { ntransfected }\end{array}$ & & & $\begin{array}{l}\text { Experimente } \\
\text { [transfected }\end{array}$ & \\
\hline Figure & $\begin{array}{c}\text { FYCO1 } \\
\text { overexpression }\end{array}$ & & 6.3 & & & 2.9 & & & & & & & \\
\hline & & & 6.6 & & & 2.1 & & & $7.2 \pm 0.6$ & & & $\begin{array}{c}2.8 \pm 0.4 \\
(p<0.0001)\end{array}$ & \\
\hline & & & 9.4 & & & 3.3 & & & & & & & \\
\hline & & $\begin{array}{l}\text { siRNA SN } \\
\text { [scramble } \\
\text { vs. siRN }\end{array}$ & $\begin{array}{l}\text { RTpool } \\
\text { control } \\
\text { pool] }\end{array}$ & $\begin{array}{r}\text { siRNA } \\
\text { [scramble } \\
\text { vs. ol }\end{array}$ & $\begin{array}{l}\text { igo } 9 \\
\text { control } \\
\text { jo] }\end{array}$ & $\begin{array}{r}\text { siRNA } 0 \\
\text { [scramble } \\
\text { vs. } 0\end{array}$ & $\begin{array}{l}\text { go } 11 \\
\text { control } \\
\text { yo] }\end{array}$ & $\begin{array}{l}\text { siRNA SM } \\
\text { [scramble } \\
\text { vs. siRN }\end{array}$ & $\begin{array}{l}\text { AARTpool } \\
\text { ed control } \\
\text { IA pool] }\end{array}$ & $\begin{array}{r}\text { siRNA } \\
\text { [scramble } \\
\text { vs. } 0\end{array}$ & $\begin{array}{l}\text { oligo } 9 \\
\text { ed control } \\
\text { oligo] }\end{array}$ & $\begin{array}{r}\text { siRNA } \\
\text { [scramble } \\
\text { vs. } 0\end{array}$ & $\begin{array}{l}\text { oligo } 11 \\
\text { ed control } \\
\text { ligo] }\end{array}$ \\
\hline Figure & FYC01 & scrambled & $\begin{array}{l}\text { siRNA } \\
\text { poof }\end{array}$ & scrambled & oligo 9 & scrambled & oligo 11 & scrambled & siRNA & scrambled & oligo 9 & scrambled & oligo 11 \\
\hline & silencing & 2.7 & 1.6 & & & & & & & & & & \\
\hline & & 4.3 & 4.0 & 4.2 & 2.5 & 2.7 & 0.8 & & & & & & \\
\hline & & 2.1 & 1.9 & 3.5 & 3.1 & 3.5 & 1.9 & $3.2 \pm 0.2$ & $\begin{array}{l}2.3 \pm 0.2 \\
(p=0.0005)\end{array}$ & $4.6 \pm 0.4$ & $\begin{array}{c}2.4 \pm 0.3 \\
(\infty<0.0001)\end{array}$ & $3.7 \pm 0.3$ & $\begin{array}{l}1.9 \pm 0.3 \\
(p=0.0001)\end{array}$ \\
\hline & & 3.2 & 1.2 & 5.9 & 1.9 & 5.9 & 4.0 & & & & & & \\
\hline Figure & $A r l 8 b^{\top 24 N}$ & [wild typ & $\begin{array}{l}\text { Control } \\
\text { Arl8b-tra }\end{array}$ & nsfected] & & $\begin{array}{l}\text { Experiment } \\
8 \mathrm{~b}^{\mathrm{T} 24 \mathrm{~N}} \text {-transf }\end{array}$ & & [wild typ & $\begin{array}{l}\text { Control } \\
\text { e Arl8b-tran }\end{array}$ & Isfected] & $\begin{array}{r}\mathrm{E} \\
{[\mathrm{Arl} 18 \mathrm{1}} \\
\end{array}$ & $\begin{array}{l}\text { Experiment } \\
b^{\mathrm{T} 24 \mathrm{~N}} \text {-transfe }\end{array}$ & ected] \\
\hline $3.6 \mathrm{C}$ & overexpression & & 9.4 & & & 3.0 & & & & & & & \\
\hline & & & 5.2 & & & 0.3 & & & $8.0 \pm 0.9$ & & & $2.8 \pm 0.5$ & \\
\hline & & & 7.7 & & & 3.3 & & & & & & & \\
\hline & & $\begin{array}{r}\text { siRNA SM } \\
\text { [scramble } \\
\text { vs. siRN } \\
\end{array}$ & $\begin{array}{l}\text { RTpool } \\
\text { control } \\
\text { pool] } \\
\end{array}$ & $\begin{array}{r}\text { siRNA o } \\
\text { [scramble } \\
\text { vs. ol } \\
\end{array}$ & $\begin{array}{l}\text { go } 10 \\
\text { control } \\
\text { jo] }\end{array}$ & $\begin{array}{r}\text { siRNA } 0 \\
\text { [scramble } \\
\text { vs. } 0 \\
\end{array}$ & $\begin{array}{l}\text { go } 12 \\
\text { control } \\
\text { jo] }\end{array}$ & $\begin{array}{r}\text { siRNA SM } \\
\text { [scramble } \\
\text { vs. siRN } \\
\end{array}$ & $\begin{array}{l}\text { AARTpool } \\
\text { ad control } \\
\text { A pool] }\end{array}$ & $\begin{array}{r}\text { siRNA } \\
\text { [scramble } \\
\text { vs. } \\
\end{array}$ & $\begin{array}{l}\text { oligo } 10 \\
\text { ed control } \\
\text { oligo] }\end{array}$ & $\begin{array}{r}\text { siRNA } \\
\text { [scramble } \\
\text { vs. } 0 \\
\end{array}$ & $\begin{array}{l}\text { oligo } 12 \\
\text { ed control } \\
\text { oligo] }\end{array}$ \\
\hline $\begin{array}{c}\text { Figure } \\
36 \mathrm{~F}\end{array}$ & $\begin{array}{c}\text { Arl8b } \\
\text { silencing }\end{array}$ & Scrambled & $\begin{array}{l}\text { siRNA } \\
\text { pool }\end{array}$ & scrambled & oligo 10 & scrambled & oligo 12 & scrambled & $\begin{array}{c}\text { siRNA } \\
\text { pool }\end{array}$ & scrambled & oligo 10 & scrambled & oligo 12 \\
\hline & & 11.0 & 4.4 & 4.2 & 2.3 & 2.7 & 0.7 & & & & & & \\
\hline & & 7.7 & 2.1 & 3.5 & 1.0 & 3.5 & 2.8 & $9.6 \pm 0.6$ & $\begin{array}{c}3.0 \pm 0.4 \\
(0<0.0001)\end{array}$ & $4.7 \pm 0.5$ & $\begin{array}{c}2.0 \pm 0.2 \\
(0<00001)\end{array}$ & $3.7 \pm 0.3$ & $\begin{array}{l}1.8 \pm 0.2 \\
(0<0.0001)\end{array}$ \\
\hline & & 8.5 & 1.9 & 5.9 & 2.5 & 5.9 & 2.7 & & & & & & \\
\hline & & $\begin{array}{r}\text { siRNA SM } \\
\text { [scramble } \\
\text { vs. siRN }\end{array}$ & $\begin{array}{l}\text { RTpool } \\
\text { control } \\
\text { pool] } \\
\end{array}$ & $\begin{array}{r}\text { siRNA } \\
\text { [scramble } \\
\text { vs. ol } \\
\end{array}$ & $\begin{array}{l}\text { igo } 5 \\
\text { control } \\
\text { o] }\end{array}$ & $\begin{array}{r}\text { siRNA } \\
\text { [scramble } \\
\text { vs. } 0 \\
\end{array}$ & $\begin{array}{l}\text { igo } 7 \\
\text { control } \\
\text { jo] }\end{array}$ & $\begin{array}{r}\text { siRNA SM } \\
\text { [scramble } \\
\text { vs. siRN } \\
\end{array}$ & $\begin{array}{l}\text { AARTpool } \\
\text { d control } \\
\text { A pool] }\end{array}$ & $\begin{array}{r}\text { siRNA } \\
\text { [scramble } \\
\text { vs. } \\
\end{array}$ & $\begin{array}{l}\text { oligo } 5 \\
\text { ed control } \\
\text { oligo] }\end{array}$ & $\begin{array}{r}\text { siRNA } \\
\text { [scramble } \\
\text { vs. } 0 \\
\end{array}$ & $\begin{array}{l}\text { oligo } 7 \\
\text { d control } \\
\text { ligo] }\end{array}$ \\
\hline $\begin{array}{l}\text { Figure } \\
3.6 \mathrm{H}\end{array}$ & $\begin{array}{c}\text { SKIP } \\
\text { silencing }\end{array}$ & Scrambled & $\begin{array}{l}\text { siRNA } \\
\text { pool }\end{array}$ & scrambled & oligo 5 & scrambled & oligo 7 & scrambled & $\begin{array}{c}\text { siRNA } \\
\text { pool }\end{array}$ & scrambled & oligo 5 & scrambled & oligo 7 \\
\hline & & 2.1 & 0.5 & 4.7 & 1.9 & 4.7 & 1.7 & & & & & & \\
\hline & & 2.1 & 0.1 & 12.2 & 6.0 & 12.2 & 5.0 & $3.1 \pm 0.3$ & $\begin{array}{c}0.8 \pm 0.2 \\
(p<0.0001)\end{array}$ & $7.7 \pm 0.7$ & $\begin{array}{l}3.8 \pm 0.5 \\
(p<0.0001)\end{array}$ & $7.7 \pm 0.7$ & $\begin{array}{l}4.0 \pm 0.6 \\
(p<0.0001)\end{array}$ \\
\hline & & 3.0 & 0.7 & 6.9 & 3.9 & 6.9 & 5.5 & & & & & & \\
\hline
\end{tabular}


Figure A-1: MRT67307, IRAK1/4 Inhibitor and PepinhMYD Inhibit Pathways Downstream of their Targets. (A) Western blot of RAW whole-cell lysates from conditions indicated above the lanes. Note that LPS induces phospho-IRF3, while pre-treatment with the TBK1 inhibitor MRT67307 followed by LPS stimulation blocked phosphorylation of IRF3, the downstream substrate of TBK1. GAPDH was probed as a loading control. (B) Relative expression of IL-6 mRNA in RAW cells treated with DMSO as control or pre-treated for 20 min with DMSO or IRAK1/4 inhbitor followed by $2 \mathrm{~h}$ LPS. Note the strong induction of IL- 6 transcription with LPS and the block in IL- 6 transcription with the IRAK1/4 inhibitor. Data are mean \pm SEM from 3 individual experiments where expression is relative to LPS condition. (C) Western blot of RAW whole-cell lysates. Cells were incubated with either Pepinh-Ctrl or Pepinh-MYD for $3 \mathrm{~h}$ followed by LPS stimulation for $2 \mathrm{~h}$. MyD88 inhibition with Pepinh-MYD strongly blocks phosphorylation of Akt in response to LPS. GAPDH was probed as a loading control.

Figure A-1

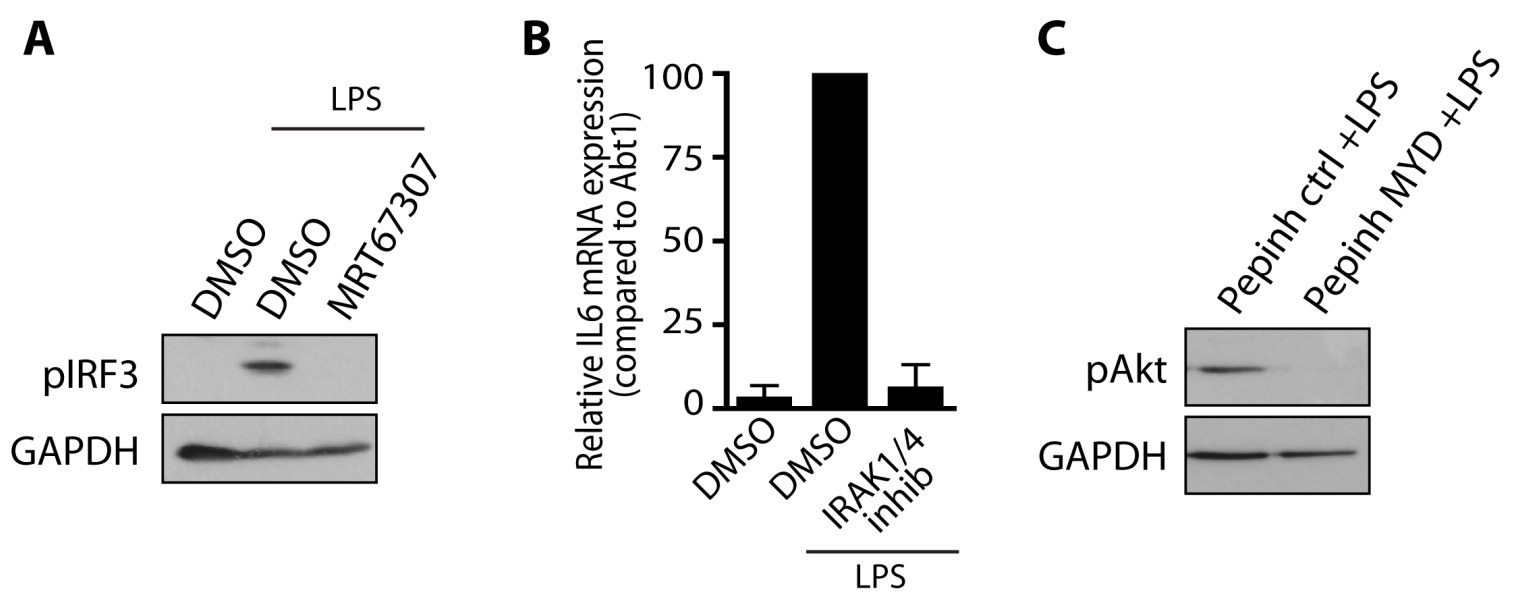


Figure A-2: mTOR Activity is Required for Lysosome Tubulation. (A) RAW cells were treated with DMSO or LPS for $2 \mathrm{~h}$ followed by gluteraldehyde fixation. Cells were immunostained for the lysosomal marker LAMP1 (red) and mTOR (green). (B) Multiple mTOR inhibitors block lysosome tubulation. Quantification of lysosome tubulation in RAW cells either treated with DMSO as control, or pre-treated for 20 min with DMSO, $1 \mu$ M rapamycin, $200 \mathrm{nM}$ PP242 or $50 \mathrm{nM}$ WYE687 followed by 100 $\mathrm{ng} / \mathrm{mL}$ LPS for $2 \mathrm{~h}$ to induce lysosome tubulation. Data are mean \pm SEM of three independent experiments based on 25-30 cells per condition per experiment. Data was statistically analyzed using a one-way ANOVA, followed by Tukey's post-hoc test. An asterisk $\left({ }^{*}\right)$ indicates a significant difference $(p<0.0001)$ between DMSO+LPS and DMSO alone. (C) Western blot of RAW whole-cell lysates showing that siRNA suppresses mTOR protein levels by at least 60\% (simTOR) relative to non-targeting siRNA oligonucleotides (siNTP). GAPDH was probed as a loading control. (D) Western blot of RAW whole-cell lysates showing that treatment of RAW cells with $100 \mu \mathrm{M}$ of the AMPK activator A769662 inhibits mTOR. Note the lack of pS6K in A769662 + LPS condition. Total S6K was probed as a loading control. 
Figure A-2

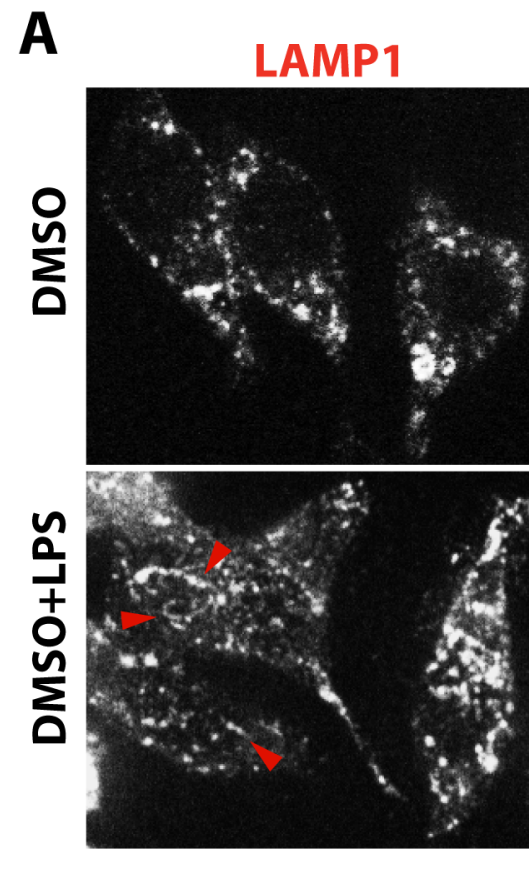

B

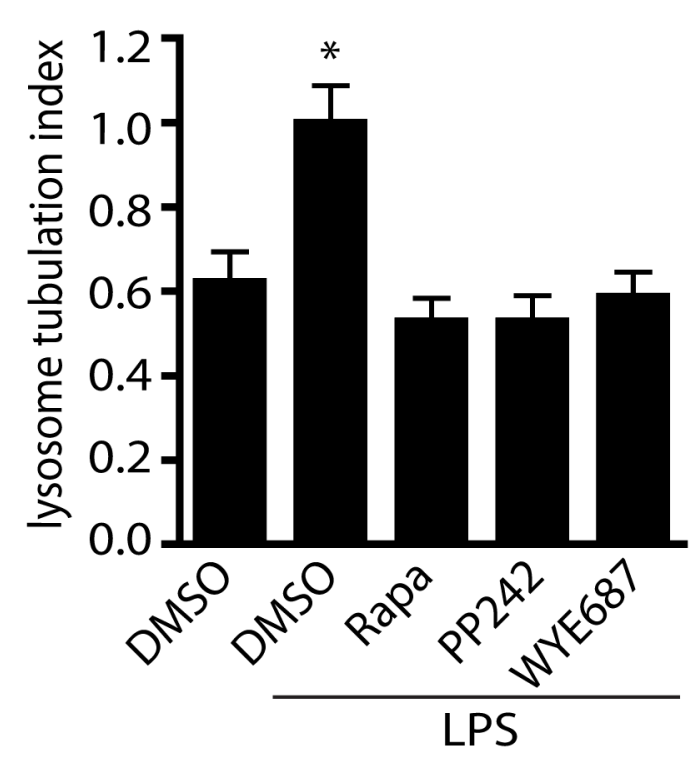

mTOR
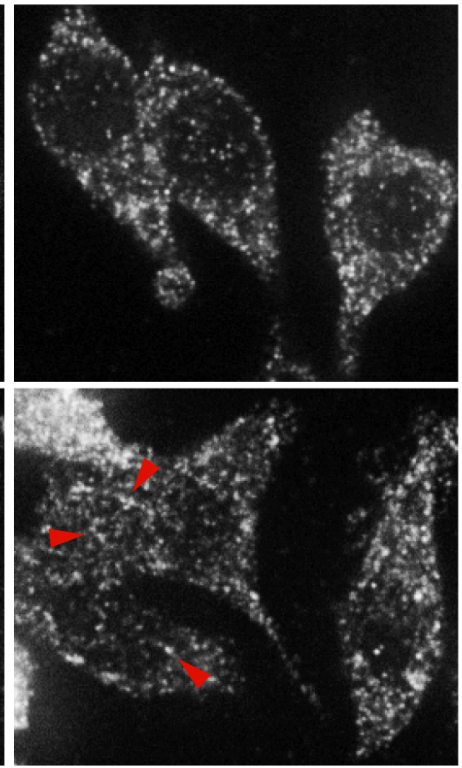

C
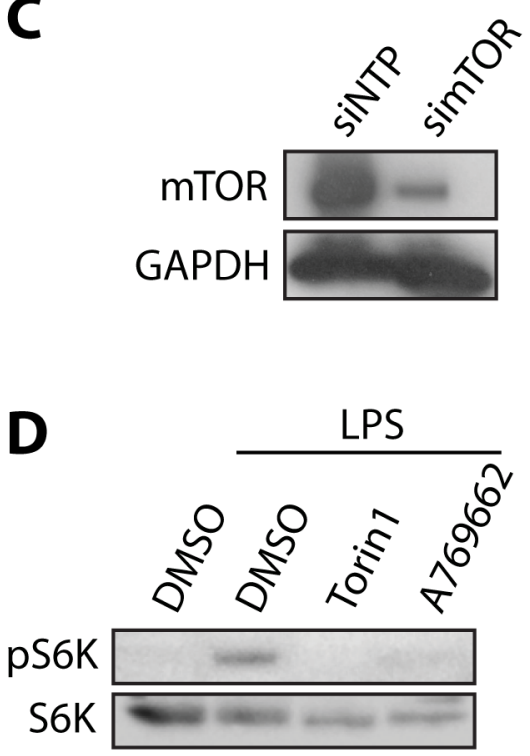
Figure A-3: mTOR Inhibition does not Affect Microtubules or Basal Lysosomal Motility. (A) Microtubules of RAW cells were stained by immunofluorescence using antibodies against $\alpha$-tubulin. Cells were either untreated, treated with $100 \mathrm{ng} / \mathrm{mL}$ LPS or $100 \mathrm{nM}$ torin1 or both for $2 \mathrm{~h}$. (B) Lysosome track length was manually followed in control (DMSO) and torin1-treated cells over a period of $1 \mathrm{~min}$ and then the lysosome speed was calculated. Each dot is an individual lysosome, with a total of 55 and 44 lysosomes from 15 cells across three independent experiments. We could not observe a difference in resting lysosome motility between control and mTOR-inhibited cells.

\section{Figure $A-3$}

A

-Torin1
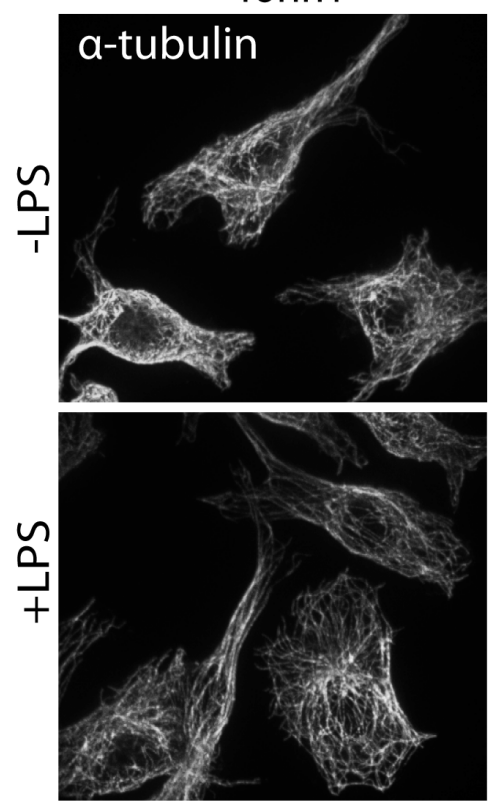

\section{+Torin1}
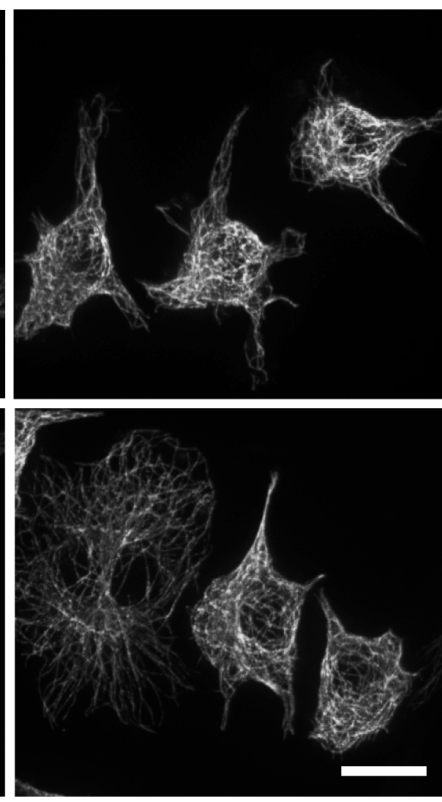

B

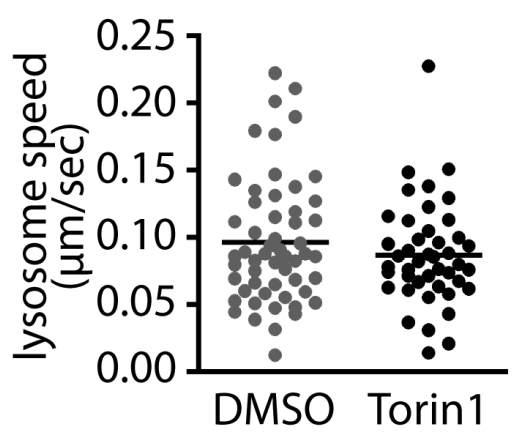


References 
Abrahamson, D. R., \& Rodewald, R. (1981). Evidence for the sorting of endocytic vesicle contents during the receptor-mediated transport of IgG across the newborn rat intestine. The Journal of Cell Biology, 91(1), 270-80.

Aeder, S. E., Martin, P. M., Soh, J.-W., \& Hussaini, I. M. (2004). PKC-eta mediates glioblastoma cell proliferation through the Akt and mTOR signaling pathways. Oncogene, 23(56), 9062-69.

Alayev, A., Salamon, R. S., Berger, S. M., Schwartz, N. S., Cuesta, R., Snyder, R. B., \& Holz, M. K. (2015). mTORC1 directly phosphorylates and activates ER $\alpha$ upon estrogen stimulation. Oncogene, 1-9. doi: 10.1038/onc.2015.414

Anderson, R. G. W., Brown, M. S., Beisiegel, U., \& Goldstein, J. L. (1982). Surface distribution and recycling of the low density lipoprotein receptor as visualized with antireceptor antibodies. The Journal of Cell Biology, 93(3), 523-31.

Askew, D., Gatewood, J., Olivas, E., Havenith, K., \& Walker, W. S. (1995). A subset of splenic macrophages process and present native antigen to naive antigenspecific CD4+ T-cells from mice transgenic for an alpha beta T-cell receptor. Cellular Immunology, 166(1), 62-70.

Bagshaw, R. D., Callahan, J. W., \& Mahuran, D. J. (2006). The Arf-family protein, Arl8b, is involved in the spatial distribution of lysosomes. Biochemical and Biophysical Research Communications, 344(4), 1186-91.

Banchereau, J., \& Steinman, R. M. (1998). Dendritic cells and the control of immunity. Nature, 392(6673), 245-52.

Bar-Peled, L., Schweitzer, L. D., Zoncu, R., \& Sabatini, D. M. (2012). Ragulator is a GEF for the rag GTPases that signal amino acid levels to mTORC1. Cell, 150(6), 1196-1208.

Barois, N., de Saint-Vis, B., Lebecque, S., Geuze, H. J., \& Kleijmeer, M. J. (2002). MHC class II compartments in human dendritic cells undergo profound structural changes upon activation. Traffic, 3(12), 894-905.

Bauerfeld, C. P., Ruchi, R., Pirockinaite, G., Lee, I., Hüttemann, M., Monks, B., Birnbaum, M. J., Franchi, L., Nunez, G., Samavati, L. (2012). TLR4-mediated AKT activation is MyD88/TRIF dependent and critical for induction of oxidative phosphorylation and mitochondrial transcription factor $\mathrm{A}$ in murine macrophages. The Journal of Immunology, 188(6), 2847-57.

Beemiller, P., Zhang, Y., Mohan, S., Levinsohn, E., Gaeta, I., Hoppe, A. D., \& Swanson, J. A. (2010). A Cdc42 activation cycle coordinated by PI 3-Kinase during Fc receptor-mediated phagocytosis. Molecular Biology of the Cell, 21(3), 470-80. 
Beg, A. A., Finco, T. S., Nantermet, P. V., \& Baldwin, A. S. J. (1993). Tumor necrosis factor and interleukin-1 lead to phosphorylation and loss of I kappa B alpha: a mechanism for NF-kappa B activation. Molecular and Cellular Biology, 13(6), 3301-10.

Behnia, R., \& Munro, S. (2005). Organelle identity and the signposts for membrane traffic. Nature, 438(7068), 597-604.

Bellacosa, A., Chan, T. O., Ahmed, N. N., Datta, K., Malstrom, S., Stokoe, D., McCormick, F., Feng, J., Tsichlis, P. (1998). Akt activation by growth factors is a multiple-step process: the role of the PH domain. Oncogene, 17(3), 313-25.

Beron, W., Alvarez-Dominguez, C., Mayorga, L., \& Stahl, P. D. (1995). Membrane trafficking along the phagocytic pathway. Trends in Cell Biology, 5(3), 100-104.

Bertho, N., Cerny, J., Kim, Y.-M., Fiebiger, E., Ploegh, H., \& Boes, M. (2003). Requirements for T cell-polarized tubulation of class II+ compartments in dendritic cells. Journal of Immunology, 171(11), 5689-96.

Beugnet, A., Tee, A. R., Taylor, P. M., \& Proud, C. G. (2003). Regulation of targets of mTOR (mammalian target of rapamycin) signalling by intracellular amino acid availability. The Biochemical Journal, 372(Pt 2), 555-66.

Boes, M., Bertho, N., Cerny, J., Op den Brouw, M., Kirchhausen, T., \& Ploegh, H. (2003). T cells induce extended class II MHC compartments in dendritic cells in a Toll-like Receptor-dependent manner. Journal of Immunology, 171(8), 408188.

Boes, M., Cerny, J., Massol, R., Op den Brouw, M., Kirchhausen, T., Chen, J., \& Ploegh, H. L. (2002). T-cell engagement of dendritic cells rapidly rearranges MHC class II transport. Nature, 418(6901), 983-8.

Boucrot, E., Henry, T., Borg, J.-P., Gorvel, J.-P., \& Meresse, S. (2005). The intracellular fate of Salmonella depends on the recruitment of kinesin. Science, 308(5725), 1174-78.

Bucci, C., Thomsen, P., Nicoziani, P., McCarthy, J., \& van Deurs, B. (2000). Rab7: a key to lysosome biogenesis. Molecular Biology of the Cell, 11(2), 467-80.

Burkhardt, J. K., Echeverri, C. J., Nilsson, T., \& Vallee, R. B. (1997). Overexpression of the dynamitin (p50) subunit of the dynactin complex disrupts dyneindependent maintenance of membrane organelle distribution. Journal of Cell Biology, 139(2), 469-84. 
Burkhardt, J. K., Hester, S., Lapham, C. K., \& Argon, Y. (1990). The lytic granules of natural killer cells are dual-function organelles combining secretory and prelysosomal compartments. Journal of Cell Biology, 111(6), 2327-40.

Burns, K., Janssens, S., Brissoni, B., Olivos, N., Beyaert, R., \& Tschopp, J. (2003). Inhibition of Interleukin 1 Receptor/Toll-like Receptor signaling through the alternatively spliced, short form of MyD88 is due to its failure to recruit IRAK-4. The Journal of Experimental Medicine, 197(2), 263-8.

Cantalupo, G., Alifano, P., Roberti, V., Bruni, C. B., \& Bucci, C. (2001). Rab-interacting lysosomal protein (RILP): the Rab7 effector required for transport to lysosomes. EMBO Journal, 20(4), 683-93.

Cantley, L. C. (2002). The phosphoinositide 3-kinase pathway. Science, 296(5573), 1655-7.

Cella, M., Engering, A., Pinet, V., Pieters, J., Lanzavecchia, A. (1997). Inflammatory stimuli induce accumulation of MHC class II complexes on dendritic cells. Nature, 388(6644), 782-7.

Cella, M., Sallusto, F., \& Lanzavecchia, A. (1997). Origin, maturation and antigen presenting function of dendritic cells. Current Opinion in Immunology, 9(1), 106.

Chavrier, P., \& Goud, B. (1999). The role of ARF and Rab GTPases in membrane transport. Current Opinion in Cell Biology, 11(4), 466-75.

Chow, A., Toomre, D., Garrett, W., \& Mellman, I. (2002). Dendritic cell maturation triggers retrograde $\mathrm{MHC}$ class II transport from lysosomes to the plasma membrane. Nature, 418(6901), 988-94.

Chung, J., Kuo, C. J., Crabtree, G. R., \& Blenis, J. (1992). Rapamycin-FKBP specifically blocks growth-dependent activation of and signaling by the $70 \mathrm{kd}$ S6 protein kinases. Cell, 69(7), 1227-36.

Cordonnier, M.-N., Dauzonne, D., Louvard, D., \& Coudrier, E. (2001). Actin filaments and myosin I alpha cooperate with microtubules for the movement of lysosomes. Molecular Biology of the Cell, 12(12), 4013-29.

Cresswell, P. (1996). Invariant chain structure and MHC class II function. Cell, 84(4), 505-7.

Cuervo, A. M., \& Dice, J. F. (2000). When lysosomes get old. Experimental Gerontology, 35(2), 119-31. 
Datta, S. R., Brunet, A., \& Greenberg, M. E. (1999). Cellular survival: a play in three Akts. Genes and Development, 13(22), 2905-27.

Dazert, E., \& Hall, M. N. (2011). mTOR signaling in disease. Current Opinion in Cell Biology, 23(6), 744-55.

Echeverri, C. J., Paschal, B. M., Vaughan, K. T., \& Vallee, R. B. (1996). Molecular characterization of the $50-\mathrm{kD}$ subunit of dynactin reveals function for the complex in chromosome alignment and spindle organization during mitosis. The Journal of Cell Biology, 132(4), 617-33.

Falcón-Pérez, J. M., Nazarian, R., Sabatti, C., \& Dell'Angelica, E. C. (2005). Distribution and dynamics of Lamp1-containing endocytic organelles in fibroblasts deficient in BLOC-3. Journal of Cell Science, 118(Pt 22), 5243-55.

Feldman, M. E., Apsel, B., Uotila, A., Loewith, R., Knight, Z. A., Ruggero, D., \& Shokat, K. M. (2009). Active-site inhibitors of mTOR target rapamycin-resistant outputs of mTORC1 and mTORC2. PLoS Biology, 7(2), e38.

Fitzgerald, K. A., Rowe, D. C., Barnes, B. J., Caffrey, D. R., Visintin, A., Latz, E., Monks, B., Pitha, P. M., Golenbock, D. T. (2003). LPS-TLR4 signaling to IRF-3/7 and NFkappa B involves the toll adapters TRAM and TRIF. The Journal of Experimental Medicine, 198(7), 1043-55.

Flannagan, R. S., Jaumouillé, V., \& Grinstein, S. (2012). The cell biology of phagocytosis. Annual Review of Pathology: Mechanisms of Disease, 7(1), 61-98.

Frasa, M. A., Maximiano, F. C., Smolarczyk, K., Francis, R. E., Betson, M. E., Lozano, E., Goldenring, J., Seabra, M. C., Rak, A., Ahmadian, M. R., Braga, V. M. (2010). Armus is a Rac1 effector that inactivates Rab7 and regulates E-cadherin degradation. Current Biology, 20(3), 198-208.

Gahl, W. A. (1989). Lysosomal membrane transport in cellular nutrition. Annual Review of Nutrition, 9, 39-61. DOI: 10.1146/annurev.nu.09.070189.000351

Garg, S., Sharma, M., Ung, C., Tuli, A., Barral, D. C., Hava, D. L., Veerapen, N., Besra, G. S., Hacohen, N., Brenner, M. B. (2011). Lysosomal trafficking, antigen presentation, and microbial killing are controlled by the Arf-like GTPase Arl8b. Immunity, 35(2), 182-93.

Gary, J. D., Wurmser, A. E., Bonangelino, C. J., Weisman, L. S., \& Emr, S. D. (1998). Fab1p is essential for PtdIns(3)P 5-kinase activity and the maintenance of vacuolar size and membrane homeostasis. The Journal of Cell Biology, 143(1), 65-79. 
Grosshans, B. L., Ortiz, D., \& Novick, P. (2006). Rabs and their effectors: achieving specificity in membrane traffic. Proceedings of the National Academy of Sciences, 103(32), 11821-7.

Gwinn, D. M., Shackelford, D. B., Egan, D. F., Mihaylova, M. M., Mery, A., Vasquez, D. S., Turk, B. E., Shaw, R. J. (2008). AMPK phosphorylation of raptor mediates a metabolic checkpoint. Molecular Cell, 30(2), 214-26.

Hardie, D. G., Ross, F. A., \& Hawley, S. A. (2012). AMPK: a nutrient and energy sensor that maintains energy homeostasis. Nature Reviews Molecular Cell Biology, 13(4), 251-62.

Harrison, R. E., Bucci, C., Vieira, O. V., Schroer, T. A., \& Grinstein, S. (2003).

Phagosomes fuse with late endosomes and / or lysosomes by extension of membrane protrusions along microtubules : role of Rab7 and RILP. Molecular and Cellular Biology, 23(18), 6494-6506.

Hartmann, B., He, X., Keller, F., Fischereder, M., Guba, M., \& Schmid, H. (2013). Development of a sensitive phospho-p70 S6 kinase ELISA to quantify mTOR proliferation signal inhibition. Therapeutic Drug Monitoring, 35(2), 233-9.

Heuser, J. (1989). Changes in lysosome shape and distribution correlated with changes in cytoplasmic pH. Journal of Cell Biology, 108(3), 855-64.

Hirokawa, N. (1998). Kinesin and dynein superfamily proteins and the mechanism of organelle transport. Science, 279(5350), 519-26.

Hirokawa, N., Noda, Y., Tanaka, Y., \& Niwa, S. (2009). Kinesin superfamily motor proteins and intracellular transport. Nature Reviews Molecular Cell Biology, 10(10), 682-96.

Hochreiter-Hufford, A., \& Ravichandran, K. S. (2013). Clearing the dead: apoptotic cell sensing, recognition, engulfment, and digestion. Cold Spring Harbor Perspectives in Biology, 5(1), 1-20.

Hoffmann, E., Marion, S., Mishra, B. B., John, M., Kratzke, R., Ahmad, S. F., Holzer, D., Anand, P. K., Weiss, D. G., Griffiths, G., Kuznetsov, S. A. (2010). Initial receptorligand interactions modulate gene expression and phagosomal properties during both early and late stages of phagocytosis. European Journal of Cell Biology, 89(9), 693-704.

Hofmann, I., \& Munro, S. (2006). An N-terminally acetylated Arf-like GTPase is localised to lysosomes and affects their motility. Journal of Cell Science, $119(\mathrm{Pt}$ 8), 1494-1503. 
Hollenbeck, P. J., \& Swanson, J. A. (1990). Radial extension of macrophage tubular lysosomes supported by kinesin. Nature, 346(6287), 864-66.

Hurley, J. H., \& Meyer, T. (2001). Subcellular targeting by membrane lipids. Current Opinion in Cell Biology, 13(2), 146-52.

Husebye, H., Halaas, Ø., Stenmark, H., Tunheim, G., Sandanger, Ø., Bogen, B., Brech, A., Latz, E., Espevik, T. (2006). Endocytic pathways regulate Toll-like receptor 4 signaling and link innate and adaptive immunity. The EMBO Journal, 25(4), 683-92.

Ikonomov, O. C., Sbrissa, D., \& Shisheva, A. (2001). Mammalian cell morphology and endocytic membrane homeostasis require enzymatically active phosphoinositide 5-kinase PIKfyve. The Journal of Biological Chemistry, 276(28), 26141-7.

Inaba, K., Inaba, M., Romani, N., Aya, H., Deguchi, M., Ikehara, S., Muramatsu, S., Steinman, R. M. (1992). Generation of large numbers of dendritic cells from mouse bone marrow cultures supplemented with granulocyte/macrophage colony-stimulating factor. The Journal of Experimental Medicine, 176(6), 16931702.

Inoki, K., Li, Y., Xu, T., \& Guan, K.-L. (2003). Rheb GTpase is a direct target of TSC2 GAP activity and regulates mTOR signaling. Genes and Development, 17(15), 1829-34.

Inoki, K., Li, Y., Zhu, T., Wu, J., \& Guan, K.-L. (2002). TSC2 is phosphorylated and inhibited by Akt and suppresses mTOR signalling. Nature Cell Biology, 4(9), 648-57.

Inoki, K., Zhu, T., \& Guan, K.-L. (2003). TSC2 mediates cellular energy response to control cell growth and survival. Cell, 115(5), 577-90.

Isotani, S., Hara, K., Tokunaga, C., Inoue, H., Avruch, J., \& Yonezawa, K. (1999). Immunopurified mammalian target of rapamycin phosphorylates and activates p70 S6 kinase alpha in vitro. The Journal of Biological Chemistry, 274(48), 34493-8.

Johansson, M., Rocha, N., Zwart, W., Jordens, I., Janssen, L., Kuijl, C., Olkkonen, V. M., Neefjes, J. (2007). Activation of endosomal dynein motors by stepwise assembly of Rab7-RILP-p150Glued, ORP1L, and the receptor $\beta$ III spectrin. Journal of Cell Biology, 176(4), 459-71.

Jordens, I., Fernandez-Borja, M., Marsman, M., Dusseljee, S., Janssen, L., Calafat, J., Janssen, H., Wubbolts, R., Neefjes, J. (2001). The Rab7 effector protein RILP 
controls lysosomal transport by inducing the recruitment of dynein-dynactin motors. Current Biology, 11(21), 1680-5.

Jung, C. H., Jun, C. B., Ro, S. H., Kim, Y. M., Otto, N. M., Cao, J., Kundu, M., Kim, D. H. (2009). ULK-Atg13-FIP200 complexes mediate mTOR signaling to the autophagy machinery. Molecular Biology of the Cell, 20(7), 1992-2003.

Jung, C. H., Ro, S. H., Cao, J., Otto, N. M., Kim, D. H. (2010). mTOR regulation of autophagy. FEBS Letters, 584(7), 1287-95.

Kaniuk, N. A., Canadien, V., Bagshaw, R. D., Bakowski, M., Braun, V., Landekic, M., Mitra, S., Huang, J., Heo, W. D., Meyer, T., Pelletier, L., Andrews-Polymenis, H., McClelland, M., Pawson, T., Grinstein, S., Brumell, J. H. (2011). Salmonella exploits Arl8B-directed kinesin activity to promote endosome tubulation and cell-to-cell transfer. Cellular Microbiology, 13(11), 1812-23.

Kardon, J. R., \& Vale, R. D. (2009). Regulators of the cytoplasmic dynein motor. Nature Reviews Molecular Cell Biology, 10(12), 854-65.

Katzmann, D. J., Babst, M., Emr, S. D. (2001). Ubiquitin-dependent sorting into the multivesicular body pathway requires the function of a conserved endosomal protein sorting complex, ESCRT-I. Cell, 106(2), 145-55.

Kawai, T., Adachi, O., Ogawa, T., Takeda, K., \& Akira, S. (1999). Unresponsiveness of MyD88-deficient mice to endotoxin. Immunity, 11(1), 115-122.

Kawai, T., Takeuchi, O., Fujita, T., Inoue, J., Mühlradt, P. F., Sato, S., Hoshino, K., Akira, S. (2001). Lipopolysaccharide stimulates the MyD88-independent pathway and results in activation of IFN-regulatory factor 3 and the expression of a subset of lipopolysaccharide-inducible genes. Journal of Immunology, 167(10), 5887-94.

Khatter, D., Raina, V. B., Dwivedi, D., Sindhwani, A., Bahl, S., \& Sharma, M. (2015). The small GTPase Arl8b regulates assembly of the mammalian HOPS complex on lysosomes. Journal of Cell Science, 128(9), 1746-61.

Kimura, S., Fujita, N., Noda, T., \& Yoshimori, T. (2009). Monitoring autophagy in mammalian cultured cells through the dynamics of LC3. In J. N. Abelson \& M. I. Simon (Eds.), Methods in Enzymology (Vol. 452, pp. 1-12). California, USA: Elsevier Inc.

Krajcovic, M., Krishna, S., Akkari, L., Joyce, J. A., \& Overholtzer, M. (2013). mTOR regulates phagosome and entotic vacuole fission. Molecular Biology of the Cell, 24(23), 3736-45. 
Laird, M. H. W., Rhee, S. H., Perkins, D. J., Medvedev, A. E., Piao, W., Fenton, M. J., \& Vogel, S. N. (2009). TLR4/MyD88/PI3K interactions regulate TLR4 signaling. Journal of Leukocyte Biology, 85(6), 966-77.

Lamb, C. A., Yoshimori, T., Tooze, S. A. (2013). The autophagosome: origins unknown, biogenesis complex. Nature Reviews Molecular Cell Biology, 14(12), $759-74$.

Laplante, M., \& Sabatini, D. M. (2013). mTOR signaling in growth control and disease. Cell, 149(2), 274-93.

Lin, S. X. H., \& Collins, C. A. (1992). Immunolocalization of cytoplasmic dynein to lysosomes in cultured cells. Journal of Cell Science, 101(Pt 1), 125-37.

Liu, Q., Chang, J. W., Wang, J., Kang, S. A., Thoreen, C. C., Markhard, A., Hur, W., Zhang, J., Sim, T., Sabatini, D. M., Gray, N. S. (2010). Discovery of 1-(4-(4propionylpiperazin-1-yl)-3-(trifluoromethyl)phenyl)-9-(quinolin-3yl)benzo[h][1,6]naphthyridin-2(1H)-one as a highly potent, selective mammalian target of rapamycin (mTOR) inhibitor for the treatment of cancer. Journal of Medicinal Chemistry, 53(19), 7146-55.

Loewith, R., Jacinto, E., Wullschleger, S., Lorberg, A., Crespo, J. L., Bonenfant, D., Oppliger, W., Jenoe, P., Hall, M. N. (2002). Two TOR complexes, only one of which is rapamycin sensitive, have distinct roles in cell growth control. Molecular Cell, 10(3), 457-68.

Luzio, J. P., Pryor, P. R., \& Bright, N. A. (2007). Lysosomes: fusion and function. Nature Reviews Molecular Cell Biology, 8(8), 622-32.

Lye, R. J., Porter, M. E., Scholey, J. M., \& McIntosh, J. R. (1987). Identification of a microtubule-based cytoplasmic motor in the nematode C. elegans. Cell, 51(2), 309-18.

Mantegazza, A. R., Zajac, A. L., Twelvetrees, A., Holzbaur, E. L. F., Amigorena, S., \& Marks, M. S. (2014). TLR-dependent phagosome tubulation in dendritic cells promotes phagosome cross-talk to optimize MHC-II antigen presentation. Proceedings of the National Academy of Sciences, 111(43), 15508-13.

Melkonian, K. A., Maier, K. C., Godfrey, J. E., Rodgers, M., \& Schroer, T. A. (2007). Mechanism of dynamitin-mediated disruption of dynactin. The Journal of Biological Chemistry, 282(27), 19355-64.

Michelet, X., Garg, S., Wolf, B. J., Tuli, A., Ricciardi-castagnoli, P., \& Brenner, M. B. (2015). MHC class II presentation is controlled by the lysosomal small GTPase, Arl8b. Journal of Immunology, 194(5), 2079-88. 
Miller, K., Beardmore, J., Kanety, H., Schlessinger, J., \& Hopkins, C. R. (1986). Localization of the epidermal growth factor (EGF) receptor within the endosome of EGF-stimulated epidermoid carcinoma (A431) cells. The Journal of Cell Biology, 102(2), 500-9.

Mizushima, N. (2007). Autophagy: process and function. Genes and Development, 21(22), 2861-73.

Mizushima, N., Yoshimori, T., \& Levine, B. (2010). Methods in mammalian autophagy research. Cell, 140(3), 313-26.

Mrakovic, A., Kay, J. G., Furuya, W., Brumell, J. H., \& Botelho, R. J. (2012). Rab7 and Arl8 GTPases are necessary for lysosome tubulation in macrophages. Traffic, 13(12), 1667-79.

Muta, T., \& Takeshige, K. (2001). Essential roles of CD14 and lipopolysaccharidebinding protein for activation of toll-like receptor (TLR)2 as well as TLR4: Reconstitution of TLR2- and TLR4-activation by distinguishable ligands in LPS preparations. European Journal of Biochemistry, 268(16), 4580-9.

Nakae, I., Fujino, T., Kobayashi, T., Sasaki, A., Kikko, Y., Fukuyama, M., Gengyo-Ando, K., Mitani, S., Kontani, K., Katada, T. (2010). The Arf-like GTPase Arl8 mediates delivery of endocytosed macromolecules to lysosomes in Caenorhabditis elegans. Molecular Biology of the Cell, 21(14), 2434-42.

Nakamura, N., Lill, J. R., Phung, Q., Jiang, Z., Bakalarski, C., de Mazière, A., Klumperman, J., Schlatter, M., Delamarre, L., Mellman, I. (2014). Endosomes are specialized platforms for bacterial sensing and NOD2 signalling. Nature, 509(7499), 240-4.

Neefjes, J. (1999). CIIV, MIIC and other compartments for MHC class II loading. European Journal of Immunology, 29(5), 1421-5.

Neefjes, J. J., Stollorz, V., Peters, P. J., Geuze, H. J., \& Ploegh, H. L. (1990). The biosynthetic pathway of MHC class II but not class I molecules intersects the endocytic route. Cell, 61(1), 171-83.

Ni, Y., Wang, L., Zhang, J., Pang, Z., Liu, Q., \& Du, J. (2015). PKD1 is downregulated in non-small cell lung cancer and mediates the feedback inhibition of mTORC1S6K1 axis in response to phorbol ester. The International Journal of Biochemistry \& Cell Biology, 60, 34-42. doi: 10.1016/j.biocel.2014.12.018.

Nordmann, M., Cabrera, M., Perz, A., Brocker, C., Ostrowicz, C., Engelbrecht-Vandre, S., Ungermann, C. (2010). The Mon1-Ccz1 complex is the GEF of the late endosomal Rab7 homolog Ypt7. Current Biology, 20(18), 1654-9. 
O'Neill, L. A., \& Bowie, A. G. (2007). The family of five: TIR domain-containing adaptors in Toll-like receptor signaling. Nature Reviews Immunology 7(5), 35364.

Pankiv, S., Alemu, E. A., Brech, A., Bruun, J. A., Lamark, T., Øvervatn, A., Bjorkoy, G., Johansen, T. (2010). FYC01 is a Rab7 effector that binds to LC3 and PI3P to mediate microtubule plus end - directed vesicle transport. The Journal of Cell Biology, 188(2), 253-69.

Pause, A., Belsham, G. J., Gingras, A.-C., Donzé, O., Lin, T.-A., Lawrence, J. C. J., \& Sonenberg, N. (1994). Insulin-dependent stimulation of protein synthesis by phosphorylation of a regulator of 5'-cap function. Nature, 371(6500), 762-7.

Peralta, E. R., Martin, B. C., Edinger, A. L. (2010). Differential effects of TBC1D15 and mammalian Vps39 on Rab7 activation state, lysosomal morphology, and growth factor dependence. Journal of Biological Chemistry, 285(22), 168814-21.

Peters, P. J., Borst, J., Oorschot, V., Fukuda, M., Krähenbühl, O., Tschopp, J., Slot, J. W., Geuze, H. J. (1991). Cytotoxic T lymphocyte granules are secretory lysosomes, containing both perforin and granzymes. The Journal of Experimental Medicine, 173(5), 1099-109.

Poteryaev, D., Fares, H., Bowerman, D., Spang, A. (2007). Caenorhabditis elegans SAND-1 is essential for Rab7 function in endosomal traffic. EMBO Journal, 26(2), 301-12.

Pu, J., Schindler, C., Jia, R., Jarnik, M., Backlund, P., \& Bonifacino, J. S. (2015). BORC, a multisubunit complex that regulates lysosome positioning. Developmental Cell, 33(2), 176-88.

Rink, J., Ghigo, E., Kalaidzidis, Y., \& Zerial, M. (2005). Rab conversion as a mechanism of progression from early to late endosomes. Cell, 122(5), 735-49.

Robinson, J. M., Chiplonkar, J., \& Luo, Z. (1996). A method for co-localization of tubular lysosomes and microtubules in macrophages: fluorescence microscopy of individual cells. Journal of Histochemistry \& Cytochemistry, 44(10), 1109-14.

Robinson, J. H., \& Delvig, A. A. (2002). Diversity in MHC class II antigen presentation. Immunology, 105(3), 252-62.

Roche, P. A., \& Cresswell, P. (1990). Invariant chain association with HLA-DR molecules inhibits immunogenic peptide binding. Nature, 345(6276), 615-8.

Rosa-Ferreira, C., \& Munro, S. (2011). Arl8 and SKIP act together to link lysosomes to kinesin-1. Developmental Cell, 21(6), 1171-8. 
Saitoh, M., Pullen, N., Brennan, P., Cantrell, D., Dennis, P. B., \& Thomas, G. (2002). Regulation of an activated S6 kinase 1 variant reveals a novel mammalian target of rapamycin phosphorylation site. The Journal of Biological Chemistry, 277(22), 20104-12.

Sancak, Y., Peterson, T. R., Shaul, Y. D., Lindquist, R. A., Thoreen, C. C., Bar-Peled, L., \& Sabatini, D. M. (2008). The Rag GTPases bind raptor and mediate amino acid signaling to mTORC1. Science, 320(5882), 1496-1501.

Sarbassov, D. D., Guertin, D. A., Ali, S. M., \& Sabatini, D. M. (2005). Phosphorylation and regulation of Akt/PKB by the rictor-mTOR complex. Science, 307(5712), 1098-1101.

Scheffzek, K., Ahmadian, M. R., Kabsch, W., Wiesmuller, L., Lautwein, A., Schmitz, F., Wittinghofer, A. (1997). The Ras-RasGAP complex: structural basis for GTPase activation and its loss in oncogenic Ras mutants. Science, 277(5324), 333-8.

Schiefermeier, N., Scheffler, J. M., de Araujo, M. E. G., Stasyk, T., Yordanov, T., Ebner, H. L., Offterdinger, M., Munck, S., Hess, M. W., Wickstrom, S. A., Lange, A., Wunderlich, W., Fassler, R., Teis, D., Huber, L. A. (2014). The late endosomal p14-MP1 (LAMTOR2/3) complex regulates focal adhesion dynamics during cell migration. Journal of Cell Biology, 205(4), 525-40.

Sengupta, S., Peterson, T. R., \& Sabatini, D. M. (2010). Regulation of the mTOR complex 1 pathway by nutrients, growth factors, and stress. Molecular Cell, $40(2), 310-22$.

Settembre, C., Fraldi, A., Medina, D. L., \& Ballabio, A. (2013). Signals from the lysosome: a control centre for cellular clearance and energy metabolism. Nature Reviews Molecular Cell Biology, 14(5), 283-96.

Settembre, C., Zoncu, R., Medina, D. L., Vetrini, F., Erdin, S., Erdin, S., Huynh, T., Ferron, M., Karsenty, G., Vellard, M. C., Facchinetti, V., Sabatini, D. M., Ballabio, A. (2012). A lysosome-to-nucleus signalling mechanism senses and regulates the lysosome via mTOR and TFEB. The EMBO Journal, 31(5), 1095-1108.

Shimazu, R., Akashi, S., Ogata, H., Nagai, Y., Fukudome, K., Miyake, K., Kimoto, M. (1999). MD-2, a molecule that confers lipopolysaccharide responsiveness on Toll-like receptor 4. Journal of Experimental Medicine, 189(11), 1777-82.

Shpetner, H. S., Paschal, B. M., \& Vallee, R. B. (1988). Characterization of the microtubule-activated ATPase of brain cytoplasmic dynein (MAP 1C). Journal of Cell Biology, 107(3), 1001-9. 
Silver, K. E., \& Harrison, R. E. (2011). Kinesin 5B is necessary for delivery of membrane and receptors during Fc $\gamma \mathrm{R}$-mediated phagocytosis. Journal of Immunology, 186(2), 816-25.

Sloan, V. S., Cameron, P., Porter, G., Gammon, M., Amaya, M., Mellins, E., Zaller, D. M. (1995). Mediation by HLA-DM of dissociation of peptides from HLA-DR. Nature, 375(6534), 802-6.

Stambolic, V., Suzuki, A., De la Pompa, J. L., Brothers, G. M., Mirtsos, C., Sasaki, T., Ruland, J., Penninger, J. M., Siderovski, D. P., Mak, T. W. (1998). Negative regulation of PKB/Akt-dependent cell survival by the tumor suppressor PTEN. Cell, 95(1), 29-39.

Steinman, R. M., \& Hemmi, H. (2006). Dendritic cells: translating innate to adaptive immunity. Curr Top Microbiol Immunol, 311, 17-58.

Steinman, R. M., \& Swanson, J. (1995). The endocytic activity of dendritic cells. The Journal of Experimental Medicine, 182(2), 283-8.

Stephen, T. L., Fabri, M., Groneck, L., Röhn, T. A., Hafke, H., Robinson, N., Rietdorf, J., Schrama, D., Becker, J. C., Plum, G., Kronke, M., Kropshofer, H., Kalka-Moll, W. M. (2007). Transport of Streptococcus pneumoniae capsular polysaccharide in MHC class II tubules. PLoS Pathogens, 3(3), e32.

Stromhaug, E. E., Berg, T. O., Fengsrud, M., Seglen, P. O. (1998). Purification and characterization of autophagosomes from rat hepatocytes. Biochemical Journal, 335(Pt 2), 217-24.

Sun-Wada, G.-H., Tabata, H., Kawamura, N., Aoyama, M., \& Wada, Y. (2009). Direct recruitment of H+-ATPase from lysosomes for phagosomal acidification. Journal of Cell Science, 122(Pt 14), 2504-13.

Suzuki, N., Suzuki, S., Duncan, G. S., Millar, D. G., Wada, T., Mirtsos, C., Takada, H., Wakeham, A., Itie, A., Li, S., Penninger, J. M., Wesche, H., Ohashi, P. S., Mak, T. W., Yeh, W.-C. (2002). Severe impairment of interleukin-1 and Toll-like receptor signalling in mice lacking IRAK-4. Nature, 416(6882), 750-6.

Swanson, J. A., Locke, A., Ansel, P., \& Hollenbeck, P. J. (1992). Radial movement of lysosomes along microtubules in permeabilized macrophages. Journal of Cell Science, 103(Pt 1), 201-9.

Swanson, J. A., Yirinec, B. D., \& Silverstein, S. C. (1985). Phorbol esters and horseradish peroxidase stimulate pinocytosis and redirect the flow of pinocytosed fluid in macrophages. The Journal of Cell Biology, 100(3), 851-9. 
Swanson, J., Burke, E., \& Silverstein, S. C. (1987). Tubular lysosomes accompany stimulated pinocytosis in macrophages. Journal of Cell Biology, 104(5), 121722.

Swanson, J., Bushnell, A., \& Silverstein, S. C. (1987). Tubular lysosome morphology and distribution within macrophages depend on the integrity of cytoplasmic microtubules. Proceedings of the National Academy of Sciences, 84(7), 1921-5.

Swiech, L., Blazejczyk, M., Urbanska, M., Pietruszka, P., Dortland, B. R., Malik, A. R., Wulf, P. S., Hoogenraad, C. C., Jaworski, J. (2011). CLIP-170 and IQGAP1 cooperatively regulate dendrite morphology. The Journal of Neuroscience, 31(12), 4555-68.

Tanaka, Y., Kanai, Y., Okada, Y., Nonaka, S., Takeda, S., Harada, A., \& Hirokawa, N. (1998). Targeted disruption of mouse conventional kinesin heavy chain, kif5B, results in abnormal perinuclear clustering of mitochondria. Cell, 93(7), 114758.

Thomas, J. A., Allen, J. L., Tsen, M., Dubnicoff, T., Danao, J., Liao, X. C., Cao, Z., Wasserman, S. A. (1999). Impaired cytokine signaling in mice lacking the IL-1 receptor-associated kinase. The Journal of Immunology, 163(2), 978-84.

Tjelle, T. E., Lovdal, T., \& Berg, T. (2000). Phagosome dynamics and function. Bioessays, 22(3), 255-63.

Tooze, S. A. \& Yoshimori, T. (2010). The origin of the autophagosomal membrane. Nature Cell Biology, 12(9), 831-5.

Vale, R. D. (2003). The molecular motor toolbox for intracellular transport. Cell, 112(4), 467-80.

Vale, R. D., Reese, T. S., \& Sheetz, M. P. (1985). Identification of a novel forcegenerating protein, kinesin, involved in microtubule-based motility. Cell, 42(1), 39-50.

Vieira, O. V., Bucci, C., Harrison, R. E., Trimble, W. S., Lanzetti, L., Gruenberg, J., Schreiber, A. D., Stahl, P. D., Grinstein, S. (2003). Modulation of Rab5 and Rab7 recruitment to phagosomes by phosphatidylinositol 3-kinase. Molecular and Cellular Biology, 23(7), 2501-14.

Von Manteuffel, S. R., Gingras, A.-C., Ming, X.-F., Sonenberg, N., \& Thomas, G. (1996). 4E-BP1 phosphorylation is mediated by the FRAP-p70s6k pathway and is independent of mitogen-activated protein kinase. Proceedings of the National Academy of Sciences, 93(9), 4076-80. 
Vyas, J. M., Kim, Y.-M., Artavanis-Tsakonas, K., Love, J. C., Van der Veen, A. G., \& Ploegh, H. L. (2007). Tubulation of class II MHC compartments is microtubule dependent and involves multiple endolysosomal membrane proteins in primary dendritic cells. Journal of Immunology, 178(11), 7199-210.

Wang, T., Ming, Z., Xiaochun, W., \& Hong, W. (2011). Rab7: role of its protein interaction cascades in endo-lysosomal traffic. Cellular Signalling, 23(3), 51621.

Watts, C. (1997). Capture and processing of exogenous antigens for presentation on MHC molecules. Annual Reviews in Immunology, 15, 821-50.

Wullschleger, S., Loewith, R., \& Hall, M. N. (2006). TOR signaling in growth and metabolism. Cell, 124(3), 471-84.

Wurmser, A. E., Sato, T. K., \& Emr, S. D. (2000). New component of the vacuolar class C-Vps complex couples nucleotide exchange on the Ypt7 GTPase to SNAREdependent docking and fusion. Journal of Cell Biology, 151(3), 551-62.

Yamamoto, M., Sato, S., \& Hemmi, H. (2003). Role of adaptor TRIF in the MyD88independent Toll-like receptor signaling pathway. Science, 301(5633), 640-3.

Yamamoto, M., Sato, S., Hemmi, H., Uematsu, S., Hoshino, K., Kaisho, T., Sanjo, H., Takeuchi, O., Sugiyama, M., Okabe, M., Takeda, K., Akira, S. (2003). TRAM is specifically involved in the Toll-like receptor 4-mediated MyD88-independent signaling pathway. Nature Immunology, 4(11), 1144-50.

Yamamoto, M., Sato, S., Mori, K., Hoshino, K., Takeuchi, O., Takeda, K., \& Akira, S. (2002). Cutting edge: a novel Toll/IL-1 receptor domain-containing adapter that preferentially activates the IFN-beta promoter in the Toll-like receptor signaling. Journal of Immunology, 169(12), 6668-72.

Yamamoto, M., \& Takeda, K. (2010). Current views on toll-like receptor signaling pathways. Gastroenterology Research and Practice, 2010, 1-8. doi: 10.1155/2010/240365.

Yang, J., \& Shen, M. H. (2006). Polyethylene glycol-mediated cell fusion. In S. Pells (Ed.), Nuclear Reprogramming (pp.59-66). Totowa, New Jersey: Humana Press.

Yrlid, U., \& Wick, M. J. (2002). Antigen presentation capacity and cytokine production by murine splenic dendritic cell subsets upon Salmonella encounter. Journal of Immunology, 169(1), 108-16.

Yu, K., Toral-Barza, L., Shi, C., Zhang, W. G., Lucas, J., Shor, B., Kim, J., Verheijen, J., Curran, K., Malwitz, D. J., Cole, D. C., Ellingboe, J., Ayral-Kaloustian, S., Mansour, T. S., Gibbons, J. J., Abraham, R. T., Nowak, P., Zask, A. (2009). Biochemical, 
cellular, and in vivo activity of novel ATP-competitive and selective inhibitors of the mammalian target of rapamycin. Cancer Research, 69(15), 6232-40.

Yu, L., McPhee, C. K., Zheng, L., Mardones, G. A., Rong, Y., Peng, J., Mi, N., Zhao, Y., Liu, Z., Wan, F., Hailey, D. W., Oorschot, V., Klumperman, J., Baehrecke, E. H., Lenardo, M. J. (2010). Autophagy termination and lysosome reformation regulated by mTOR. Nature, 465(7300), 942-6.

Zoncu, R., Bar-Peled, L., Efeyan, A., Wang, S., Sancak, Y., \& Sabatini, D. M. (2011). mTORC1 senses lysosomal amino acids through an inside-out mechanism that requires the vacuolar H-ATPase. Science, 334(6056), 678-83.

Zoncu, R., Sabatini, D. M., \& Efeyan, A. (2011). mTOR: from growth signal integration to cancer, diabetes and ageing. Nature Reviews Molecular Cell Biology, 12(1), 21-35. 